\title{
Uso de Métodos MCMC para Análise Bayesiana de Dados de Sobrevivência na Presença de Covariáveis
}

\author{
Cillene da Silva Nunes de Souza
}

Orientador: Prof. Dr. Jorge Alberto Achcar

Dissertação apresentada ao Instituto de Ciências Matemáticas e de Computação - ICMC-USP, como parte dos requisitos para obtenção do título de Mestre em Ciências de Computação e Matemática Computacional.

USP - São Carlos

Março/2001 


\section{A Comissão Julgadora:}

Prof. Dr. Jorge Alberto Achcar

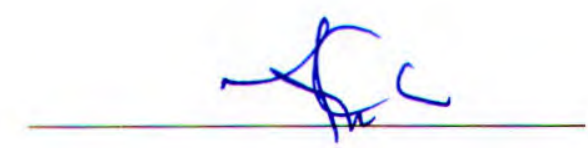

Prof. Dr. Marinho Gomes de Andrade Filho

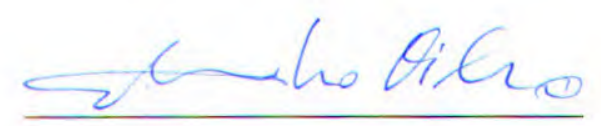

Prof. Dr. Ronaldo Dias

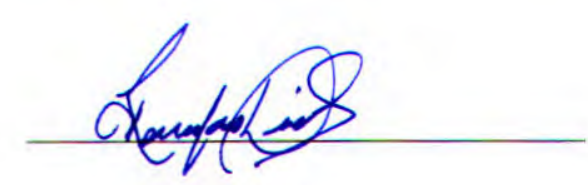




\section{Agradecimentos}

Agradeço em primeiro lugar a Deus e a todos os espíritos de luz que estão presentes sob várias formas e em todas as etapas de minha vida.

Aos meus pais, Elcio e Diva, que sempre acreditaram no meu trabalho e me deram apoio moral e financeiro para eu permanecer em São Carlos. Agradeço também aos meus irmãos, Elcio e Cibelle, cunhados, Jos e Valéria e ao meu sobrinho Tiago por entender minha atenção parcial neste período.

Ao Professor Dr. Jorge Alberto Achcar pela atenção, pela orientação segura e por todo o incentivo durante esses dois anos.

Ao Professor Dr. Marinho de Andrade Filho pela amizade e incentivo nas disciplinas, pelas valiosas sugestões no Exame de Qualificação e pela força para lutar por um laboratório para a Estatística.

Ao Professor Dr. Francisco Louzada Neto pela maneira educada e gentil em fazer críticas e dar sugestões importantes no Exame de Qualificação, as quais contribuíram muito com essa dissertação.

Ao amigo e quase doutor Josmar Mazucheli por me apresentar softwares novos e eficazes, pela paciência e companhia nesses anos.

Aos amigos de turma Emerson, Juliano, Sidney, Manuel e Ulisses sem os quais o mestrado não teria a menor graça e pela amizade valiosa.

Às minhas amigas, ‘irmãs' e companheiras Dayse e Valéria por tudo que passamos juntas.

Às minhas grandes amigas Alessandra e Josiane por estarem sempre comigo nos momentos dificeis e por me desculparem a ausência em momentos importantes.

Aos meus amigos Sandra, Rúbia, Fritz, Érica, Karina, Valdirene, Gilberto , Carol, Ricardo, Zé Flávio, Juan, Juanita, Roberta, Luiz Vitor, İtalo, Lucas, Poperô e Rio Claro.

Ao Wendell pelo carinho e atenção recebidos nos últimos meses.

Aos professores da UNICAMP que me incentivaram muito a fazer mestrado, em especial, ao orientador de iniciação científica e amigo Dr. Ronaldo Dias.

A todos os funcionários do ICMC, em especial às meninas da Secretaria da Pós Graduação e aos porteiros do laboratório.

Ao CNPq pelo apoio financeiro. 


\section{Resumo}

Nesta dissertação estamos interessados na análise Bayesiana de dados de sobrevivência médicos com observações censuradas e na presença de uma ou mais covariáveis. Em muitos casos, podemos assumir um modelo de regressão paramétrica para os dados como uma alternativa aos modelos de regressão não paramétricas. Em casos especiais, podemos precisar de uma regressão paramétrica com uma distribuição mais geral para os dados de sobrevivência como uma mistura de distribuiçōes. Um dos objetivos principais do projeto é relacionado ao uso de misturas de distribuições paramétricas para a variável erro nos modelos log-linerares.

No contexto Bayesiano utilizamos técnicas de simulação de Monte Carlo em Cadeias de Markov (MCMC) e destacamos a utilização dos softwares "Ox "e "WinBugs" como grandes alternativas para os problemas referente ao tempo de execução dos algoritmos computacionais. 


\section{Abstract}

In this dissertation, we develop a Bayesian analysis for survival medical data with censored observations and in the presence of one or more covariates. In many cases, we can assume a parametrical model for the lifetime data as na alternative to nonparametrical models. In many special cases, we need a parametrical regression model with a more general distribution as a mixture of distributions for the error assuming log-linear models.

In the Bayesian approach, we use MCMC simulation methods (Markov Chain Monte Carlo) using the softwares $O x$ and WinBugs as a computational alternative to other computational programs. 


\section{SUMÁRIO}

CAPÍTULO 1 : Introdução

CAPÍTULO 2: Modelos de Regressão Paramétrica $-4$

2.1 Modelos de Riscos Proporcionais 4

2.2 Modelos de Locação- Escala

2.2.1 Modelos de Regressão Exponencial

2.2.2 Modelos de Regressão Weibull e Valor Extremo

2.2.3 Modelos de Regressão Log- Logístico

2.2.4 Modelos de Regressão Log- Normal

$\longrightarrow 14$

2.2.5 Modelos de Regressão Gama Generalizada _____ 15

2.2.6 Modelos de Regressão Birbaum-Saunders ___ 18

CAPÍTULO 3: Alguns Exemplos de Aplicação de Modelos de Regressão Paramétrica_ 20

3.1 Primeiro Conjunto de Dados 20

3.1.1 Modelo de Regressão Exponencial ___ 21

3.1.2 Modelo de Regressão Weibull

3.1.3 Modelo de Regressão Logístico 23

3.1.4 Modelo de Regressão Log- Normal 25

3.2 Segundo Conjunto de Dados 27

3.2.1 Modelo de Regressão Exponencial ___ _ 29

3.2.2 Modelo de Regressão Weibull ___ 31

3.2.3 Modelo de Regressão Log - Logístico ___ 32

3.2.4 Modelo de Regressão Log - Normal _- 34

3.2.5 Modelo de Regressão Gama Generalizada___ 36

3.3 Terceiro Conjunto de Dados 38

3.3.1 Modelo de Regressão Exponencial___ 39

3.3.2 Modelo de Regressão Weibull _ـ 40

3.3.3 Modelo de Regressão Log - Logístico ___ 42

3.3.4 Modelo de Regressão Log - Normal — 43

3.3.5 Modelo de Regressão Birbaum Saunders ___ 45

3.4 Quarto Conjunto de Dados _ 46

3.4.1 Modelo de Regressão Exponencial ___ 47

3.4.2 Modelo de Regressão Weibull _ 50

3.4.3 Modelo de Regressão Logística ____ 52

3.4.4 Modelo de Regressão Log- Normal_______ 55 
CAPÍTULO 4: Mistura de Distribuições 58

4.1 Introdução aos Modelos de Mistura de Distribuições

4.1.1 Princípio Básico baseado em amostra ampliada 58

4.2 Mistura de 2 Distribuições Weibull para $T$ 59

4.3 Mistura de 3 Distribuições Weibull para T 61

4.4 Mistura de 2 Distribuições Gama Generalizadas 62

4.5 Mistura de 2 Distribuições Normais para log(T) 63

4.6 Mistura de Distribuições Normal e Exponencial 64

4.7 Exemplos de Mistura de Distribuições

4.7.1 Modelo de Mistura nos dados 2

4.7.2 Modelo de Mistura para os dados 3 66 66 67 73

CAPÍTULO 5: Comparação e Seleção de Modelos 78

5.1 Critério Clássico de seleção de modelos 78

5.2 Critério Bayesiano de seleção de modelos 79

5.3 Seleção para o primeiro conjunto de dados 80

5.4 Seleção para o segundo conjunto de dados 81

5.5 Seleção para o terceiro conjunto de dados 82

5.4 Seleção para o quarto conjunto de dados 84

CAPÍTULO 6 : Uso de Métodos Bayesianos em Modelos de Riscos Proporcionais 85

6.1 Modelos de Riscos Proporcionais 85

6.2 Modelo de Efeitos Aleatórios 86

6.3 Modelo de Efeitos Aleatórios com Mistura de Normais 87

6.4 llustração Numérica 89

CAPÍTULO 7: Conclusões e Propostas Futuras 93

ANEXO $A$ 95

ANEXO B 97

ANEXOC 99 


\section{CAPÍTULO 1 : Introdução}

Métodos estatísticos para analisar dados de ensaios médicos foram introduzidos em meados dos anos 60 . Os primeiros ensaios conduzidos em programas de grupos cooperativos foram quase todos baseados em ensaios clínicos aleatórios, e foi aceito como o primeiro método para comparar terapias. Nesses programas cooperativos, cada nova droga progredia através de uma série de ensaios médicos: a fase 1 era para se determinar a dosagem da droga, a 2 era para determinar a eficácia preliminar e a 3 para comparar os efeitos dos tratamentos ( ver Gehan e Lemak,1994). Muitos métodos para análise estatística foram introduzidos nesta época: um plano para ensaios na fase 2 por Gehan(1961), uma generalização de testes de Wilcoxon para comparar funçōes de sobrevivência com dados censurados à direita (Gehan, 1965), um teste para funções de riscos proporcionais para comparar 2 funçōes de sobrevivência (Mantel e Haenszel, 1959), aplicaçōes de funçōes de sobrevivência (Mantel, 1966) e modelo de regressão exponencial relacionando tempo de sobrevivência com variáveis explicativas (Feigl e Zelen, 1965).

Dentro deste contexto, apresentamos o nosso trabalho analisando dados médicos e usando novas técnicas com o destaque para uma mistura de distribuiçōes em inferência Bayesiana para melhorar as estimativas dos tempos de sobrevivência de pacientes com doenças terminais.

Em muitas aplicaçöes com dados de sobrevivência médicos temos observações censuradas e a presença de uma ou mais covariáveis. Em muitos casos podemos assumir como uma alternativa aos modelos de regressão não paramétrica, um modelo de regressão paramétrica para os dados. Em particular, podemos considerar uma regressão exponencial, uma regressão de Weibull ou uma regressão Gama Generalizada. Em casos especiais, podemos precisar de uma regressão paramétrica com uma distribuição mais geral para os dados de sobrevivência como uma mistura de distribuições de Weibull.

Em geral, podemos ter dificuldades para obter inferências clássicas para esse tipo de modelo na presença de várias covariáveis e dados censurados. 
O uso de métodos Bayesianos para esse tipo de modelo pode ser de grande aplicabilidade, especialmente usando métodos MCMC (Monte Carlo em Cadeias de Markov). Entre esses métodos de simulação, destacamos o método de 'adaptive rejection Metropolis sampling' (ARMS).

O uso de distribuições com misturas de distribuições de sobrevivências usuais pode ser necessário quando temos uma função de risco em forma de $U$ ou quando a distribuição de sobrevivência é multimodal.

Nesta situação podemos considerar uma mistura de distribuiçōes de Weibull ou Gama Generalizada (ver por exemplo, Diebolt \& Robert, 1994). Um caso especial é dado pela mistura de duas distribuições de Weibull com f.d.p ,

$$
f(t)=p \frac{\beta_{1}}{\alpha_{1}}\left(\frac{t}{\alpha_{1}}\right)^{\beta_{1}-1} \exp \left\{-\left(\frac{t}{\alpha_{1}}\right)^{\beta_{1}}\right\}+(1-p) \frac{\beta_{2}}{\alpha_{2}}\left(\frac{t}{\alpha_{2}}\right)^{\beta_{2}-1} \exp \left\{-\left(\frac{t}{\alpha_{2}}\right)^{\beta_{2}}\right\}
$$

Para a obtenção de quantidades a posteriori de interesse, consideramos o uso de métodos MCMC e também introduzimos variáveis latentes (ver por exemplo, Tanner \& Wong, 1987) para simplificar as distribuiçōes condicionais necessárias para o algoritmo ARMS.

No capítulo 2, introduzimos alguns modelos de regressão paramétrica loglineares. Além disso, explicitamos como fazer a análise clássica e Bayesiana para os modelos propostos. Como utilizamos esses modelos para alguns conjuntos de dados, temos algumas verossimilhanças considerando censuras e outras não, de acordo com o conjunto de dados para os quais os modelos foram desenvolvidos.

O Capítulo 3 apresenta todos as estimativas dos parâmetros dados pelos modelos introduzidos no Capítulo 2, onde temos quatro conjuntos de dados com graus de dificuldades diferentes, alguns sem covariáveis, outros com covariáveis e censuras. Além disso, é importante observar que em alguns casos trabalhamos com a transformação $Y=\log T$ e em outros casos diretamente com o tempo de sobrevivência.

No Capítulo 4, introduzimos modelos com mistura de distribuições onde 
análises Clássica e Bayesiana são feitas para esses modelos. Além disso, temos exemplos para esses modelos.

Uma introdução a seleção de modelos sob enfoque clássico e Bayesiano é apresentada no Capítulo 5. Descrevemos como utilizar o critério da densidade preditiva ordenada (CPO) no caso Bayesiano. Neste Capítulo também temos os resultados das seleçōes para cada um dos quatro conjuntos de dados.

No Capítulo 6, introduzimos um tipo de modelo diferente do restante da dissertação. Nele, utilizamos modelos de riscos proporcionais e apresentamos alguns exemplos de utilização desses modelos.

Finalmente, no capítulo 7 apresentamos algumas conclusões do trabalho como um todo, mostrando vantagens e desvantagens do uso de métodos Bayesianos.

Alguns objetivos do nosso trabalho são:

(i) Considerar modelos de regressão paramétrica para dados de sobrevivência censurados.

(ii) Uso de métodos MCMC para uma análise Bayesiana dos modelos propostos.

(iii) Considerar modelos com misturas de distribuições de sobrevivência.

(iv) Considerar métodos Bayesianos para discriminar os modelos propostos. 


\section{CAPÍTULO 2: Modelos de Regressão Paramétrica}

Quando estudamos dados de sobrevivência temos muitas situações que envolvem populaçōes heterogêneas e nelas é importante considerar a relação entre o tempo de sobrevivência com outros fatores. Um modo de fazer isto é através de modelos de regressão, nos quais a dependência do tempo de sobrevivência com algumas variáveis explanatórias é explicitado.

Um exemplo ilustrativo é dado por Krall et al (1975) que relacionam os tempos de sobrevivência de 65 pacientes com câncer com uma série de fatores e para isso consideram 16 variáveis explanatórias, incluindo medidas fisiológicas tais como a quantidade de hemoglobina no sangue e contagem de glóbulos brancos.

Neste capítulo estudamos vários modelos de regressão paramétrica como os modelos Exponencial, Weibull e Log-logístico. Vamos considerar modelos com e sem censuras nos dados.

Podemos considerar duas classes de modelos de regressão:

1) Modelos de riscos proporcionais para $T$

2) Modelos de regressão locação- escala para $T$

onde T é o tempo de sobrevivência.

\subsection{Modelos de Riscos Proporcionais}

Para descrevermos os modelos de riscos proporcionais podemos considerar a distribuição Weibull. Seja $\alpha$ um parâmetro de escala e $\delta$ um parâmetro de forma de uma distribuição Weibull. Supor que somente $\alpha$ dependa de $\mathbf{x}$, onde $\mathbf{x}$, é um vetor de covariáveis $1 \times p$,

$$
f(t \mid \mathbf{x})=\frac{\beta}{\alpha(\mathbf{x})}\left(\frac{t}{\alpha(\mathbf{x})}\right)^{\beta-1} \exp \left\{\left(-\frac{t}{\alpha(\mathbf{x})}\right)^{\beta}\right\}
$$


A função de sobrevivência é dada por:

$S(t \mid \mathbf{x})=\exp \left\{-\left(\frac{t}{\alpha(\mathbf{x})}\right)^{\beta}\right\}$

onde : 0 e $\alpha(\mathbf{x})$ é função de $\mathbf{x}$ que envolve parâmetros desconhecidos.

Portanto a função de risco é dada por,

$$
h(t \mid \mathbf{x})=-\frac{S^{\prime}(t \mid \mathbf{x})}{S(t \mid \mathbf{x})}=\frac{\beta}{\alpha(\mathbf{x})}\left(\frac{t}{\alpha(\mathbf{x})}\right)^{\beta-1}
$$

Com isso, temos que a razão de funções de risco para 2 indivíduos com covariáveis $\mathbf{x}_{1}$ e $\mathbf{x}_{2}$ é dado por:

$$
\frac{h\left(t \mid \mathbf{x}_{1}\right)}{h(t \mid \mathbf{I}}=\frac{\frac{\beta}{\alpha\left(\mathbf{x}_{1}\right)}\left(\frac{t}{\alpha\left(\mathbf{x}_{1}\right)}\right)^{\beta-1}}{\frac{\beta}{\alpha\left(\mathbf{x}_{2}\right)}\left(\frac{t}{\alpha\left(\mathbf{x}_{2}\right)}\right)^{\beta-1}}=\left(\frac{\alpha\left(\mathbf{x}_{2}\right)}{\alpha\left(\mathbf{x}_{1}\right)}\right)^{\beta}
$$

Podemos observar por (2.4) que a razão não depende do tempo de sobrevivência, isto é, indivíduos diferentes tem funçōes de risco proporcionais.

\subsection{Modelos de Locação- Escala}

Se $T$ tem distribuição Weibull, a distribuição de $Y=\log T_{1}$ dado $\mathbf{x}$ é a distribuição de valor extremo, dada na forma,

$$
\begin{aligned}
& f(y \mid \mathbf{x})=\frac{1}{\sigma} \exp \left\{\frac{y-\mu(\mathbf{x})}{\sigma}-\exp \left(\frac{y-\mu(\mathbf{x})}{\sigma}\right)\right\} \\
& \text { onde }-\infty<y<\infty, \sigma=1 / \delta \text { e } \mu(\mathbf{x})=\log \alpha(\mathbf{x})
\end{aligned}
$$

Podemos reescrever a equação (2.5) na forma, 
$Y=\mu(\mathbf{x})+\sigma Z$

onde $\mathrm{Z}$ tem uma distribuição de valor extremo padronizada com fdp

$f(z)=\exp (z-\exp (z))$

onde $-\infty<z<\infty$.

O modelo 2.6 é conhecido como modelo de locação- escala com erro $Z$.

Uma forma possivel para a dependência de $\mu$ em relação à x é dada por:

$\mu(\mathbf{x})=\mathbf{x} \boldsymbol{\beta}=\beta_{0}+\beta_{1} x_{1}+\ldots+\beta_{p} x_{p}$

onde $\mathbf{x}$ é um vetor $1 \times p$ de covariáveis e $\beta$ é um vetor $p \times 1$ dos coeficientes de regressăo.

Agora, mostraremos alguns casos especiais do modelo (2.6).

\subsubsection{Modelos de Regressão Exponencial}

Supor unidades com funçöes de riscos constantes que dependem de covariáveis. A fdp do tempo de sobrevivência Té dada por:

$f\left(t_{i} \mid \mathbf{x}\right)=\frac{1}{\theta_{\mathbf{x}_{t}}} \exp \left\{-\frac{t_{i}}{\theta_{\mathbf{x}_{l}}}\right\}$

para $t_{i}>0$ e onde $\theta_{x_{i}}$ pode ser dado pela relaçăo $\theta_{x_{1}}=\exp (\mathbf{x} \boldsymbol{\beta})$.

Assim temos,

$y_{i}=\log \left(t_{i}\right)=\mathbf{x} \boldsymbol{\beta}+Z_{i}$

onde $Z$ é uma variável erro com ${ }_{6}$ distribuição de valor extremo com fdp 
dada em (2.7).

\subsubsection{ANÁLISE CLÁSSICA}

- Dados sem censuras

A função de verossimilhança de um modelo do tipo (2.9) para dados sem censuras é dada por:

$$
\begin{aligned}
L(\boldsymbol{\beta})= & \left.\prod_{i=1}^{n}\left\{\exp \left[\left(y_{i}-\mathbf{x} \boldsymbol{\beta}\right)-\exp \left(y_{i}-\mathbf{x} \boldsymbol{\beta}\right)\right]\right\}\right] \\
& \text { Portanto, } \\
L(\boldsymbol{\beta})= & \exp \left\{\sum_{i=1}^{n} y_{i}-\sum_{i=1}^{n} \mathbf{x} \boldsymbol{\beta}\right\} \exp \left\{-\sum_{i=1}^{n} \exp \left(y_{i}-\mathbf{x} \boldsymbol{\beta}\right)\right\}
\end{aligned}
$$

Para a análise clássica deste modelo, usualmente encontramos estimadores de máxima verossimilhança para os parâmetros e usamos teoria assintótica para obter as inferências de interesse.

\section{- Dados com censuras}

Sejam $t_{i}$ os tempos de vida de $n$ unidades $i=1, \ldots, n$ e $x_{i}$ os vetores de regressão associados a cada unidade dados por:

$x_{i}=\left(x_{11}, x_{i 2}, \ldots, x_{i p}\right)$

Considere $\mathrm{D}$ representando o conjunto de unidades que falham e $\mathrm{C}$ o conjunto de unidades que sobrevivem (dados censurados) durante o experimento.

Com $Y=\log T$, a fdp de $Y$ dado o vetor $\mathbf{x}$ é dada por:

$$
f(y \mid \mathbf{x})=\exp \{(y-\mathbf{x} \boldsymbol{\beta})-\exp (y-\mathbf{x} \boldsymbol{\beta})\}
$$

Neste caso, a função de sobrevivência é dada por:

$$
S(y \mid \mathbf{x})=\exp \{-\exp (y-\mathbf{x} \boldsymbol{\beta})\}
$$


Então, a função de verossimilhança para o vetor $\beta$ pode ser escrita como:

$$
L(\boldsymbol{\beta})=\prod_{i \in D} \exp \left[\left(y_{i}-\mathbf{x} \boldsymbol{\beta}\right)-\exp \left(y_{i}-\mathbf{x} \boldsymbol{\beta}\right) \prod_{i \in C} \exp \left[-\exp \left(y_{i}-\mathbf{x} \boldsymbol{\beta}\right)\right]\right.
$$

O logaritmo da verossimilhança é dado por:

$$
l(\boldsymbol{\beta})=\sum_{i \in D}\left(y_{i}-\mathbf{x} \boldsymbol{\beta}\right)-\sum_{i=1}^{n} \exp \left(y_{i}-\mathbf{x} \boldsymbol{\beta}\right)
$$

Daí, maximizar $l(\beta)$ para encontrarmos os EMV dos parâmetros.

\subsubsection{2 -ANÁLISE BAYESIANA}

Assumindo independência a priori podemos utilizar as seguintes distribuições a priori para os parâmetros de interesse:

$$
\pi\left(\beta_{j}\right) \sim N\left(a_{j}, b_{j}{ }^{2}\right) \quad ; \text { com } \mathrm{a}_{\mathrm{j}} \text { e } \mathrm{b}_{\mathrm{j}} \text { conhecidos } \mathrm{e} \mathrm{j}=0,1, \ldots, \mathrm{p}
$$

Vamos introduzir uma análise Bayesiana para o caso geral, ou seja, com dados censurados. Observar que em caso de dados sem censuras, a verossimilhança fica simplificada e é fácil adaptá-la ao novo modelo.

A utilização do algoritmo ARMS (ver por exemplo Gilks et al. 1997) requer o logaritmo da verossimilhança condicional. Assim, o logaritmo da verossimilhança para dados censurados é dado por,

$$
l(\boldsymbol{\beta})=r \log (\sigma)+\sum_{i=1}^{r}\left(y_{i}-\mathbf{x} \boldsymbol{\beta}\right)-\sum_{i=1}^{n} \exp \left(y_{i}-\mathbf{x} \boldsymbol{\beta}\right)
$$

onde $r$ representa os dados observados. 
Os logaritmos das distribuiçōes condicionais para o algoritmo ARMS săo dadas por:

$$
\begin{gathered}
i) \pi\left(\beta_{j} \mid \boldsymbol{\beta}_{(j)}, \mathbf{x}, \mathbf{y}\right)=-\sum_{i=1}^{r} \beta_{j} x_{i j}-\sum_{i=1}^{n} \exp \left(y_{i}-\mathbf{x} \boldsymbol{\beta}\right)+\log \pi\left(\beta_{j}\right) \\
\text { onde } \mathrm{j}=0, \ldots \mathrm{p} \text { e } \boldsymbol{\beta}_{(j)}=\left(\beta_{0}, \ldots, \beta_{j-1}, \beta_{j+1}, \ldots, \beta_{p}\right) .
\end{gathered}
$$

\subsubsection{Modelos de Regressão Weibull e Valor Extremo}

Seja T uma variável aleatória não-negativa representando o tempo de vida de uma unidade com densidade Weibull dada em (2.1).

Como suposto anteriormente somente $\alpha$ depende de $\mathbf{x}$ e temos o modelo de riscos proporcionais para os tempos de sobrevivência.

Vimos também que a distribuição de $Y=\log T$ pode ser dada pela equação (2.5).

\subsubsection{ANÁLISE CLÁSSICA}

- Dados sem censuras

Quando temos dados não- censurados, a equação de verossimilhança para o modelo Weibull é dada por:

$$
L(\boldsymbol{\beta}, \sigma)=\prod_{i=1}^{n} \frac{1}{\sigma} \exp \left\{\frac{y_{i}-\mathbf{x} \boldsymbol{\beta}}{\sigma}-\exp \left(\frac{y_{i}-\mathbf{x} \boldsymbol{\beta}}{\sigma}\right)\right\}
$$

Quando consideramos apenas uma covariável $x$, isto é, $\mathbf{x} \boldsymbol{\beta}=\beta_{0}+\beta_{1} x_{1}$, a função de verossimilhança para $\boldsymbol{\beta}=\left(\beta_{0}, \beta_{1}\right)$ e $\sigma$ é dada por,

$$
L(\boldsymbol{\beta}, \sigma) \propto \frac{1}{\sigma^{n}} \exp \left\{\frac{y_{i}-\mathbf{x} \boldsymbol{\beta}}{\sigma}\right\} \exp \left\{-\sum_{i=1}^{n} \exp \left(\frac{y_{i}-\mathbf{x} \boldsymbol{\beta}}{\sigma}\right)\right\}
$$


- Dados censurados

Com dados censurados, a função de verossimithança é dada por,

$$
L(\mathbf{\beta}, \sigma)=\prod_{i \in D} \frac{1}{\sigma} \exp \left\{\frac{y_{i}-\mathbf{x} \boldsymbol{\beta}}{\sigma}-\exp \left(\frac{y_{i}-\mathbf{x} \boldsymbol{\beta}}{\sigma}\right)\right\} \prod_{i \in C} \exp \left\{-\exp \left(\frac{y_{i}-\mathbf{x} \boldsymbol{\beta}}{\sigma}\right)\right\}
$$

\subsubsection{ANÁLISE BAYESIANA}

Para desenvolver uma análise Bayesiana precisamos das distribuições a priori para $\beta$ e $\sigma$. Supor,

(i) $\sigma \sim I G(a, b)$; a, b conhecidos,

$$
\text { i.e, } \pi(\sigma) \propto \sigma^{-(a+1)} \exp \left\{-\frac{b}{\sigma}\right\}, \sigma>0
$$

(ii) $\beta_{f} \sim N\left(c_{j}, d_{j}^{2}\right) \quad, \mathrm{c}_{\mathrm{j}}, \mathrm{d}_{\mathrm{j}}$ conhecidos; $\mathrm{j}=0, \ldots, \mathrm{p}$

onde IG(a,b) denota uma distribuiçăo Gama inversa e $N\left(c_{j}, d_{j}^{2}\right)$ denota uma distribuição Normal.

O logaritmo da verossimilhança é dado por,

$$
l(\boldsymbol{\beta}, \sigma)=r \log (\sigma)+\sum_{i=1}^{r}\left(\frac{y_{i}-\mathbf{x} \boldsymbol{\beta}}{\sigma}\right)-\sum_{i=1}^{n} \exp \left(\frac{y_{i}-\mathbf{x} \boldsymbol{\beta}}{\sigma}\right)
$$

onde $r$ é o número de observações não-censuradas.

Os logaritmos das distribuições condicionais para o algoritmo ARMS são dadas por, 


$$
\begin{aligned}
& (i) \pi(\sigma \mid \boldsymbol{\beta}, \mathbf{y}, \mathbf{x})=r \log (\sigma)+\sum_{i=1}^{r}\left(\frac{y_{i}-\mathbf{x} \boldsymbol{\beta}}{\sigma}\right)-\sum_{i=1}^{n} \exp \left(\frac{y_{i}-\mathbf{x} \boldsymbol{\beta}}{\sigma}\right)+\log \pi(\sigma) \\
& (i i) \pi\left(\beta_{j} \mid \boldsymbol{\beta}_{(j)}, \mathbf{y}, \mathbf{x}\right)=-\sum_{i=1}^{r} \frac{\beta_{j} x_{i j}}{\sigma}-\sum_{i=1}^{n} \exp \left(\frac{y_{i}-\mathbf{x} \boldsymbol{\beta}}{\sigma}\right)+\log \pi\left(\beta_{j}\right)
\end{aligned}
$$

\subsubsection{Modelos de Regressáo Log- Logístico}

Um outro modelo alternativo para o modelo de regressäo Weibull é dado pelo modelo log logístico.

Neste caso, o tempo de sobrevivência $T$ tem uma distribuiçăo log- logística se o logaritmo do tempo de sobrevivência $Y=\ln T$, tiver uma distribuiçăo logística com densidade,

$$
f(y \mid \mathbf{x})=\frac{1}{\sigma} \frac{\exp \left[\left(\frac{y-x \beta}{\sigma}\right)\right]}{\left\{1+\exp \left[\left(\frac{y-x \beta}{\sigma}\right)\right]\right\}^{2}} \quad,-\infty<y<\infty
$$

Assim, assumir no modelo (2.6) que $Z$ tem uma distribuição logística padrão com densidade dada por:

$$
f(z)=\frac{\exp (z)}{[1+\exp (z)]^{2}}
$$

A funçăo de sobrevivência é dada por:

$$
S(z)=\frac{1}{[1+\exp (z)]}
$$




\subsubsection{ANÁLISE CLÁSSICA}

- Dados sem censuras

A função de verossimilhança considerando dados sem censuras é dada por:

$$
\begin{aligned}
L(\boldsymbol{\beta}, \sigma) & =\prod_{i=1}^{n} \frac{1}{\sigma} \frac{\exp \left(\frac{y_{i}-\mathbf{x} \boldsymbol{\beta}}{\sigma}\right)}{\left(1+\exp \left(\frac{y_{i}-\mathbf{x} \boldsymbol{\beta}}{\sigma}\right)\right)^{2}} \\
& =\frac{1}{\sigma^{n}} \frac{\exp \left\{\sum_{i=1}^{n}\left(\frac{y_{i}-\mathbf{x} \boldsymbol{\beta}}{\sigma}\right)\right\}}{\prod_{i=1}^{n}\left(1+\exp \left(\frac{y_{i}-\mathbf{x} \boldsymbol{\beta}}{\sigma}\right)\right)^{2}}
\end{aligned}
$$

O logaritmo da verossimilhança é dado por,

$$
l(\boldsymbol{\beta}, \sigma)=-n \log \sigma+\sum_{i=1}^{n}\left(\frac{y_{i}-\mathbf{x} \boldsymbol{\beta}}{\sigma}\right)-\sum_{i=1}^{n} 2 \log \left(1+\exp \left(\frac{y_{i}-\mathbf{x} \boldsymbol{\beta}}{\sigma}\right)\right)
$$

- Dados censurados

Para dados censurados a equaçăo de verossimilhança leva em conta a função de sobrevivência .Assim,

$$
\begin{aligned}
L(\boldsymbol{\beta}, \sigma) & =\prod_{i \in D} \frac{1}{\sigma} \frac{\exp \left(\frac{y_{i}-\mathbf{x} \boldsymbol{\beta}}{\sigma}\right)}{\left(1+\exp \left(\frac{y_{i}-\mathbf{x} \boldsymbol{\beta}}{\sigma}\right)\right)^{2}} \prod_{i \in C} \frac{1}{\left(1+\exp \left(\frac{y_{i}-\mathbf{x} \boldsymbol{\beta}}{\sigma}\right)\right)} \\
& =\frac{1}{\sigma^{x}} \frac{\exp \sum_{i \in D}\left(\frac{y_{i}-\mathbf{x} \boldsymbol{\beta}}{\sigma}\right)}{\prod_{i \in D}\left(1+\exp \left(\frac{y_{i}-\mathbf{x} \boldsymbol{\beta}}{\sigma}\right)\right)^{2}} \prod_{i \in C}\left(1+\exp \left(\frac{y_{i}-\mathbf{x \beta}}{\sigma}\right)\right)
\end{aligned}
$$

O loganitmo da verossimilhança é dado por, 


$$
l(\boldsymbol{\beta}, \sigma)=-r \log \sigma+\sum_{i \in D}\left(\frac{y_{i}-\mathbf{x} \boldsymbol{\beta}}{\sigma}\right)-2 \sum_{i \in D} \log \left(1+\exp \left(\frac{y_{i}-\mathbf{x} \boldsymbol{\beta}}{\sigma}\right)\right)-\sum_{i \in C} \log \left(1+\exp \left(\frac{y_{i}-\mathbf{x} \boldsymbol{\beta}}{\sigma}\right)\right)
$$

\subsubsection{ANÁLISE BAYESIANA}

Para uma análise Bayesiana precisamos das distribuições a priori para os parâmetros de interesse. Supor,

(i) $\sigma \sim I G(a, b)$; a, b conhecidos,

i.e, $\pi(\sigma) \propto \sigma^{-(\mathrm{a}+1)} \exp \left\{\frac{-b}{\sigma}\right\}, \sigma>0$

(ii) $\beta_{j} \sim N\left(c_{j}, d_{j}^{2}\right) ; \mathrm{c}_{\mathrm{j}}, d_{j}$ conhecidos, $\mathrm{j}=0,1,2,3,4$

onde $I G(a, b)$ denota uma distribuição Gama inversa e $N\left(c_{j}, d_{j}^{2}\right)$ denota uma distribuição Normal.

Os logaritmos das distribuiçōes condicionais para o algoritmo de ARMS baseadas na equação (2.32) são dadas por,

$$
\begin{aligned}
(i) \pi(\sigma \backslash \boldsymbol{\beta}, \mathbf{x}, \mathbf{y})= & -r \log (\sigma)+\sum_{i=1}^{r}\left(\frac{y_{i}-\mathbf{x} \boldsymbol{\beta}}{\sigma}\right)-2 \sum_{i=1}^{r} \log \left(1+\exp \left(\frac{y_{i}-\mathbf{x} \boldsymbol{\beta}}{\sigma}\right)\right) \\
& -\sum_{i \in C} \log \left(1+\exp \left(\frac{y_{i}-\mathbf{x} \boldsymbol{\beta}}{\sigma}\right)\right)+\log \pi(\sigma) \\
(i i) \pi\left(\beta_{j} \mid \boldsymbol{\beta}_{(j)}, \sigma, \mathbf{x}, \mathbf{y}\right)= & \sum_{i=1}^{r}\left(\frac{y_{i}-\mathbf{x \beta}}{\sigma}\right)-2 \sum_{i=1}^{r} \log \left(1+\exp \left(\frac{y_{i}-\mathbf{x} \boldsymbol{\beta}}{\sigma}\right)\right) \\
& -\sum_{i \in C} \log \left(1+\exp \left(\frac{y_{i}-\mathbf{x} \boldsymbol{\beta}}{\sigma}\right)\right)+\log \pi\left(\beta_{j}\right)
\end{aligned}
$$

onde $\mathrm{j}=0, \ldots \mathrm{p}$ e $\boldsymbol{\beta}_{(j)}=\left(\beta_{0}, \ldots, \beta_{j-1}, \beta_{j+1}, \ldots, \beta_{p}\right)$. 


\subsubsection{Modelos de Regressão Log- Normal}

A distribuição log-normal, assim como a distribuição de Weibull tem sido muito usada em modelos de tempo de sobrevivência. Na verdade, ela tem sido usada em diversas situações como na análise de tempos de falhas elétricas (Nelson e Hahn, 1972) e no estudo de tempos de aparecimento de câncer no pulmão em fumantes (Whittemore e Altschuler, 1976).

Essa distribuição é mais facilmente especificada assumindo que o tempo de sobrevivência $T$ é distribuído com a distribuição log-normal se o logaritmo $Y=\ln T$ é normalmente distribuído com média $\mu$ e variância $\sigma^{2}$.

Assim, assumimos que $Z$, no modelo (2.6), tem uma distribuição normal padrão com densidade,

$$
f(z)=\frac{1}{(2 \pi)^{1 / 2}} \exp \left[-\frac{1}{2} z^{2}\right] \quad,-\infty<z<\infty
$$

e função de sobrevivência,

$$
S(z)=1-F(z)=1-\phi(z)
$$

\subsubsection{ANÁLISE CLÁSSICA}

Vamos denotar $S(z)=Q(z)=\int_{z}^{\infty} \phi(x) d x$ onde $\phi(z)$ é a densidade de $Z$ dada em (2.37).

Daí, a densidade e a função de sobrevivência de $Y$ são dadas por,

$$
f(y \mid \mathbf{x})=\frac{1}{\sigma} \phi\left(\frac{\mathrm{y}_{\mathrm{i}}-\mathbf{x}_{\mathbf{1}} \boldsymbol{\beta}}{\sigma}\right) \quad,-\infty<\mathrm{y}<\infty
$$

e

$$
S(y \backslash \mathbf{x})=Q\left(\frac{y_{i}-\mathbf{x}_{\mathbf{\beta}} \boldsymbol{\beta}}{\sigma}\right)
$$


- Dados sem censuras

A equação de verossimilhança para dados sem censuras é dada por:

$$
\begin{aligned}
& L(\boldsymbol{\beta}, \sigma)=\prod_{i=1}^{n} \frac{1}{\sigma} \phi\left(\frac{y_{i}-\mathbf{x}_{1} \boldsymbol{\beta}}{\sigma}\right) \\
& \text { Daí, } \\
& l(\boldsymbol{\beta}, \sigma)=-n \log \sigma-\frac{1}{2 \sigma^{2}} \sum_{i=1}^{n}\left(y_{i}-\mathbf{x}_{i} \mathbf{\beta}\right)^{2}
\end{aligned}
$$

- Dados com censuras

No caso de dados censurados no modelo log-normal temos a função de verossimilhança,

$$
L(\boldsymbol{\beta}, \sigma)=\prod_{i \in D} \frac{1}{\sigma} \phi\left(\frac{y_{i}-\mathbf{x}_{\mathbf{i}} \mathbf{\beta}}{\sigma}\right) \prod_{i \in \mathcal{C}} Q\left(\frac{y_{i}-\mathbf{x}_{i} \boldsymbol{\beta}}{\sigma}\right)
$$

O logaritmo da verossimilhança é dado por,

$$
l(\boldsymbol{\beta}, \sigma)=-r \log \sigma-\frac{1}{2 \sigma^{2}} \sum_{i \in D}\left(y_{i}-\mathbf{x}_{1} \boldsymbol{\beta}\right)^{2}+\sum_{i \in C} \log Q\left(\frac{y_{i}-\mathbf{x}_{\mathbf{A}} \mathbf{\beta}}{\sigma}\right)
$$

\subsubsection{ANÁLISE BAYESIANA}

Para fazermos uma análise Bayesiana, vamos considerar as distribuições a priori dadas na seção 2.2.3.2. Com isso e com a equação (2.44), temos as seguintes distribuições condicionais na forma logaritmica,

$(i) \pi(\sigma \backslash \boldsymbol{\beta}, \mathbf{x}, \mathbf{y})=-r \log (\sigma)-\frac{1}{2 \sigma^{2}} \sum_{i=1}^{r}\left(y_{i}-\mathbf{x} \boldsymbol{\beta}\right)^{2}+\sum_{i \in C} \log Q\left(\frac{y_{i}-\mathbf{x} \boldsymbol{\beta}}{\sigma}\right)+\log \pi(\sigma)$

$(i i) \pi\left(\beta_{j} \mid \boldsymbol{\beta}_{(j)}, \sigma, \mathbf{x}, \mathbf{y}\right)=\frac{1}{2 \sigma^{2}} \sum_{i=1}^{r}\left(y_{i}-\mathbf{x} \boldsymbol{\beta}\right)+\sum_{i \in C} \log Q\left(\frac{y_{i}-\mathbf{x} \boldsymbol{\beta}}{\sigma}\right)+\log \pi(\beta)$

com $r \in \boldsymbol{\beta}_{(j)}$ dados nas seções anteriores.

\subsubsection{Modelos de Regressão Gama Generalizada}

A distribuição log- gama inclui casos especiais de distribuições de 
tempos de sobrevivência. Ela pode ser utilizada na discriminação entre a distribuição normal e a distribuição de valor extremo, ou equivalentemente, entre a Weibull e a log-normal.

A distribuição do logarítimo do tempo de sobrevivência $Y=\ln T$, dado um vetor $\mathbf{x}$ de covariáveis pode ser representado pelo modelo (2.6).

Neste caso, Z tem uma distribuiçäo log-gama com fdp:

$$
f(z, k)=\left\{\begin{array}{lr}
\frac{k^{k-0.5}}{\Gamma(k)} \exp \left(\sqrt{k} z-k \exp \left(\frac{z}{\sqrt{k}}\right)\right. & 0<\mathrm{k}<\infty \\
\frac{1}{(2 \pi)^{1 / 2}} \exp \left(\frac{-z^{2}}{2}\right) & \mathrm{k}=\infty
\end{array}\right.
$$

Observar que quando $k=1$ temos a distribuição VE (valor extremo) e quando $\mathrm{k} \uparrow \infty$ temos o modelo normal.

É importante lembrar que $z_{i}=\frac{y_{i}-x \boldsymbol{\beta}}{\sigma}$ pelo modelo (2.6). Com isso, fica fácil encontrar a distribuição de $Y$.

\subsubsection{ANÁLISE CLÁSSICA}

- Dados sem censuras

A função de verossimilhança é dada por,

$$
L(\boldsymbol{\beta}, \sigma, k)=\prod_{i=1}^{n^{*}} \frac{1}{\sigma} f\left(y_{i}, k\right)
$$

e o logaritmo da verossimilhança é dado por,

$$
l(\boldsymbol{\beta}, \sigma, k)=(\mathrm{k}-0.5) \log k-\log (\sigma)-\log \Gamma(k)+\sqrt{k}\left(\frac{y_{i}-\mathbf{x} \boldsymbol{\beta}}{\sigma}\right)-k e z p\left(\frac{y_{i}-\mathbf{x} \boldsymbol{\beta}}{\sigma \sqrt{k}}\right)
$$


Com dados censurados, o logaritmo da função de verossimilhança é dado por,

$l(\boldsymbol{\beta}, \sigma, k)=(\mathrm{k}-0.5) \log k-\log (\sigma)-\log \Gamma(k)+\sqrt{k}\left(\frac{y_{i}-\mathbf{x} \boldsymbol{\beta}}{\sigma}\right)-k e z p\left(\frac{y_{i}-\mathbf{x} \boldsymbol{\beta}}{\sigma \sqrt{k}}\right)+\sum_{i \in C} \log Q\left(k, k \exp \left(\frac{z_{i}}{\sqrt{k}}\right)\right.$

onde

$\mathrm{Q}(\mathrm{k}, \mathrm{a})=\int_{0}^{\infty} \frac{\mathrm{x}^{\mathrm{k}-\mathrm{l}}}{\Gamma(\mathrm{k})} \exp (-x) d x=1-\mathrm{I}(k, a)$

O modelo gama generalizada pode ser utilizado como um supermodelo para encontrar um modelo paramétrico adequado para os dados de sobrevivência. Testes de hipóteses para $k=1$ ou $k=0$ são usados para verificar se os modelos Weibull ou log-normal são apropriados.

\subsubsection{ANÁLISE BAYESIANA}

Neste modelo, consideramos as mesmas distribuiçōes a priori anteriores, porém, agora temos um novo parâmetro $(k)$ e a priori para esse parâmetro pode ser dada por,

$(i) k \sim \Gamma(a, b) ; a, b$ conhecidos,

i.e, $\pi(k)=b^{a} x^{a-1} \exp (-b x) / \Gamma(a)$

Assim, os logaritmos das distribuições condicionais são dados por,

$(i) \pi(\sigma \mid \boldsymbol{\beta}, k, \mathbf{x})=-\log (\sigma)+\sqrt{k}\left(\frac{y_{1}-\mathbf{x} \boldsymbol{\beta}}{\sigma}\right)-k e z p\left(\frac{y_{1}-\mathbf{x} \boldsymbol{\beta}}{\sigma \sqrt{k}}\right)+\sum_{i \in C} \log Q\left(k, k \exp \left(\frac{y_{1}-\mathbf{x} \boldsymbol{\beta}}{\sigma \sqrt{k}}\right)+\log \pi(\sigma)\right.$

$(i i) \pi\left(\beta_{j} \mid \boldsymbol{\beta}_{(j)}, \sigma, k, \mathbf{x}\right)=\sqrt{k}\left(\frac{y_{i}-\mathbf{x} \boldsymbol{\beta}}{\sigma}\right)-k \operatorname{ezp}\left(\frac{y_{i}-\mathbf{x} \boldsymbol{\beta}}{\sigma \sqrt{k}}\right)+\sum_{i \in C} \log Q\left(k, k \exp \left(\frac{y_{1}-\mathbf{x} \boldsymbol{\beta}}{\sigma \sqrt{k}}\right)\right)+\log \pi\left(\beta_{j}\right)$

(iii) $\pi(k \mid \boldsymbol{\beta}, \sigma)=(\mathbf{k}-0.5) \log k-\log \Gamma(k)+\sqrt{k}\left(\frac{y_{1}-\mathbf{x} \boldsymbol{\beta}}{\sigma}\right)-k e z p\left(\frac{y_{1}-\mathbf{x} \boldsymbol{\beta}}{\sigma \sqrt{k}}\right)+\sum_{i \in C} \log Q\left(k, k \exp \left(\frac{y_{1}-\mathbf{x} \boldsymbol{\beta}}{\sigma \sqrt{k}}\right)+\log \pi(k)\right.$ 


\subsubsection{Modelos de Regressão Birbaum-Saunders}

A distribuição de Birnbaum-Saunders foi derivada para modelar fadigas de objetos de metal . A distribuição para o tempo de falha é dada por, (ver Achcar, 1991)

$$
f(t ; \alpha, \beta)=\frac{\left(t^{2}-\beta^{2}\right) \exp \left\{-\frac{1}{2 \alpha^{2}}\left[\frac{t}{\beta}+\frac{\beta}{t}-2\right]\right\}}{2 \sqrt{2 \pi} \alpha \beta t^{2}\left[\left(\frac{t}{\beta}\right)^{1 / 2}-\left(\frac{\beta}{t}\right)^{1 / 2}\right]}
$$

onde $\mathrm{t}, \alpha, \beta>0$.

Os parâmetros $\alpha$ e $\beta$ são respectivamente, parâmetros de forma e escala.

Se uma variável aleatória $T$ tem distribuição Birnbaum-Saunders com parâmetros $\alpha$ e $\beta$, então a distribuição da variável aleatória $Y=\ln (T)$ tem uma distribuição seno-normal com densidade, (ver Achcar e Espinosa, 1991)

$$
f(y ; \alpha, \gamma)=\left\{\frac{2}{2 \alpha \sqrt{2 \pi}}\right\} \cosh \left(\frac{y-\gamma}{2}\right) \exp \left\{-\frac{2}{\alpha^{2}} \sinh ^{2}\left(\frac{y-\gamma}{2}\right)\right\}
$$

onde $-\infty<y<\infty, \alpha$ é o parâmetro de forma e $\gamma=\ln (\beta)$ é o parâmetro de locação.

\subsubsection{ANÁLISE CLÁSSICA}

Considerando dados sem censuras, a função de verossimilhança é dada por,

$$
L(\boldsymbol{\beta}, \sigma)=\left\{\frac{1}{2 \sqrt{2 \pi}}\right\}^{n}\left\{\prod_{i=1}^{n} \frac{2}{\alpha} \cosh \left(\frac{y-\mathbf{x} \boldsymbol{\beta}}{2}\right)\right\} \exp \left\{-\sum_{i=1}^{n} \frac{2}{\alpha^{2}} \sinh ^{2}\left(\frac{y-\mathbf{x} \boldsymbol{\beta}}{2}\right)\right\}
$$

O logaritmo da verossimilhança é, 
$l(\boldsymbol{\beta}, \sigma) \propto \sum_{i=1}^{n} \ln W_{i}-\sum_{i=1}^{n} \frac{Z_{i}^{2}}{2}$

onde $\mathrm{W}_{\mathrm{i}}=\frac{2}{\alpha} \cosh \left(\frac{y_{i}-\mathbf{x} \boldsymbol{\beta}}{2}\right)$ e $\mathrm{Z}_{\mathrm{i}}=\frac{2}{\alpha} \sinh \left(\frac{y_{i}-\mathbf{x} \boldsymbol{\beta}}{2}\right)$

\subsubsection{ANÁLISE BAYESIANA}

Neste caso, todos os termos dependem de todos os parâmetros e assim, os logaritmos das distribuições condicionais para os parâmetros de interesse são dadas pela equação (2.58) mais o logaritmo da distribuição a priori. 


\section{CAPÍTULO 3: Alguns Exemplos de Aplicação de Modelos de Regressão Paramétrica}

Neste capítulo, apresentamos alguns exemplos de aplicação de modelos de regressão paramétrica. Para isso, utilizamos os dados de Feigl \& Zelen (1965) para ilustrar problemas com uma única covariável, os dados de fadigas de materiais elétricos para ilustrar alguns tipos de mistura que estudaremos no capítulo seguinte e os dados de Kardaum (1983) cujos dados já são mais complexos e possuem várias covariáveis no modelo.

Para verificar a convergência do algoritmo Gibbs Sampling vamos utilizar o critério de Gelman \& Rubin (1992) e observar como se comportam as simulações dos parâmetros. Essas convergências foram verificadas utilizando o software CODA( ver por exemplo, Best et al., 1996). Também utilizaremos o critério de Geweke o os resultados foram obtidos no Matlab.

As estimativas de máxima verossimilhança foram feitas utilizando o software SAS $\Theta$ as Bayesianas usando o software WinBUGS12 (Gilks et al. 1994) ou OX(ver Doornik 1999), dependendo do grau de dificuldade do problema, já que no OX temos mais liberdade de programação.

\subsection{Primeiro Conjunto de Dados}

Na Tabela 3.1 temos os dados de 17 pacientes com leucemia. (Feigl e Zelen, 1965). Os tempos $t_{i}$ são os tempos de sobrevivência dados em semanas. Uma única covariável $\mathrm{x}$ é associada com cada paciente, a quantidade de glóbulos brancos (WBC) medida na hora do diagnóstico. No caso, usamos o logaritmo na base 10 dessa quantidade.

Além disso, na coluna 4 temos a variável $x_{i}^{\prime}$, que é dada pelo valor de $x_{i}$ menos a média dos valores, com isso a soma de $x_{i}^{\prime} e$ igual a zero. Essa transformação sugerida por Lawless (1982) . 
Tabela 3.1 Tempos de Sobrevivência para 17 pacientes com Leucemia

\begin{tabular}{c|c|c|c}
\hline $\mathbf{t}_{\mathbf{i}}$ & $\mathbf{W B C}$ & $\mathbf{x}_{\mathbf{i}}$ & $\mathbf{x}_{\mathbf{i}}{ }^{\prime}$ \\
\hline 65 & 2300 & 3,36 & $-0,735$ \\
\hline 156 & 750 & 2,88 & $-1,215$ \\
\hline 100 & 4300 & 3,63 & $-0,465$ \\
\hline 134 & 2600 & 3,41 & $-0,685$ \\
\hline 16 & 6000 & 3,78 & $-0,315$ \\
\hline 108 & 10000 & 4,02 & $-0,095$ \\
\hline 121 & 10000 & 4,00 & $-0,095$ \\
\hline 4 & 17000 & 4,23 & 0,135 \\
\hline 39 & 5400 & 3,73 & $-0,365$ \\
\hline 143 & 7000 & 3,85 & $-0,245$ \\
\hline 56 & 9400 & 3,97 & $-0,125$ \\
\hline 26 & 32000 & 4,51 & 0,415 \\
\hline 22 & 35000 & 4,54 & 0,445 \\
\hline 1 & 100000 & 5,00 & 0,905 \\
\hline 1 & 100000 & 5,00 & 0,905 \\
\hline 5 & 52000 & 4,72 & 0,625 \\
\hline 65 & 100000 & 5,00 & 0,905 \\
\hline & & &
\end{tabular}

\subsubsection{Modelo de Regressão Exponencial}

Assumimos primeiramente o modelo de regressão exponencial (2.9) para os dados da Tabela 3.1.

Para esses dados temos $\mathbf{x} \boldsymbol{\beta}$ igual à $\beta_{0}+\beta_{1} x$, já que temos apenas uma covariável no modelo; isso será utilizado em todos os modelos ajustados para esses dados.

Através do modelo exponencial, obtivemos as estimativas clássicas e Bayesianas para os parâmetros $\beta_{0}$ e $\beta_{1}$ apresentadas na Tabela 3.2 abaixo. Maiores detalhes como as priori utilizadas podem ser encontrados na seção 2.1.1. Os 
valores considerados para os hiperparâmetros das distribuições a priori são : $a_{j}=0, b_{j}=1000 .(j=0,1)$

Tabela 3.2 Estimativas para o modelo de regressăo exponencial

\begin{tabular}{c|c|c|c|c}
\hline Parâmetro & EMV & $\begin{array}{c}\text { Intervalo de } \\
\text { Confiança } \\
(95 \%)\end{array}$ & $\begin{array}{c}\text { Média a } \\
\text { posteriori }\end{array}$ & $\begin{array}{c}\text { Intervalo de } \\
\text { Credibilidade } \\
(95 \%)\end{array}$ \\
\hline$\beta_{0}$ & 3,934 & $(3,458 ; 4.409)$ & 3,987 & $(3,500 ; 4,473)$ \\
\hline$\beta_{1}$ & $-1,109$ & $(-1,920 ;-0.298)$ & $-1,087$ & $(-1,845 ;-0,329)$ \\
\hline$\sigma^{*}=1$ & & - & & - \\
\hline
\end{tabular}

Podemos notar que para o modelo de regressão exponencial conseguimos estimativas próximas com a abordagem clássica e Bayesiana. Não tivemos nenhuma dificuldade para encontrar essas estimativas, no caso Bayesiano os resultados foram obtidos através do software WinBUGS.

$\mathrm{Na}$ Tabela 3.3, temos os valores que indicam convergência das cadeias para os dois parâmetros, usando o critério de Gelman e Rubin para avaliar a convergência (ver Gelman e Rubin, 1992).

Tabela 3.3: Valores obtidos para $\sqrt{R}$ no critério de convergência de Gelman e Rubin para os parâmetros.

\begin{tabular}{c|c}
\hline & $\sqrt{R}$ \\
\hline$\beta_{0}$ & 1,000 \\
\hline$\beta_{1}$ & 1,000 \\
\hline
\end{tabular}

Com $\sqrt{R} \approx 1,00$, a convergência do algoritmo é verificada.

Na Figura 3.1 temos os gráficos das distribuiçōes a posteriori marginais dos parâmetros, e gráficos de auto- correlação e gráficos para monitorar as cadeias para cada parâmetro respectivamente.
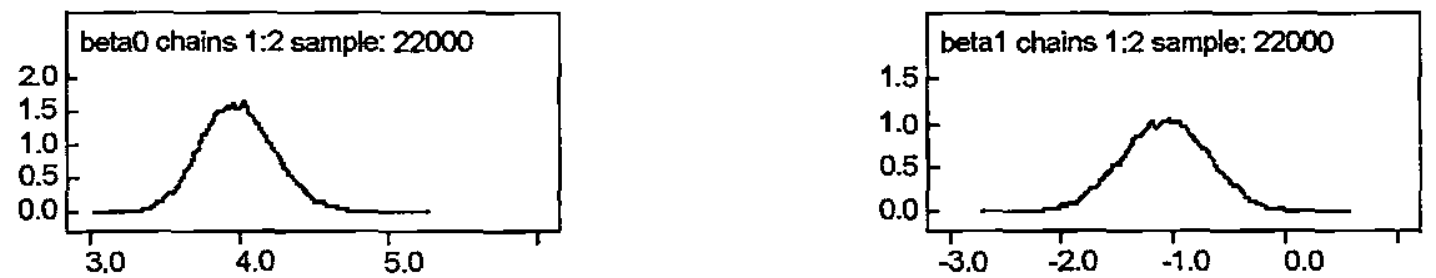

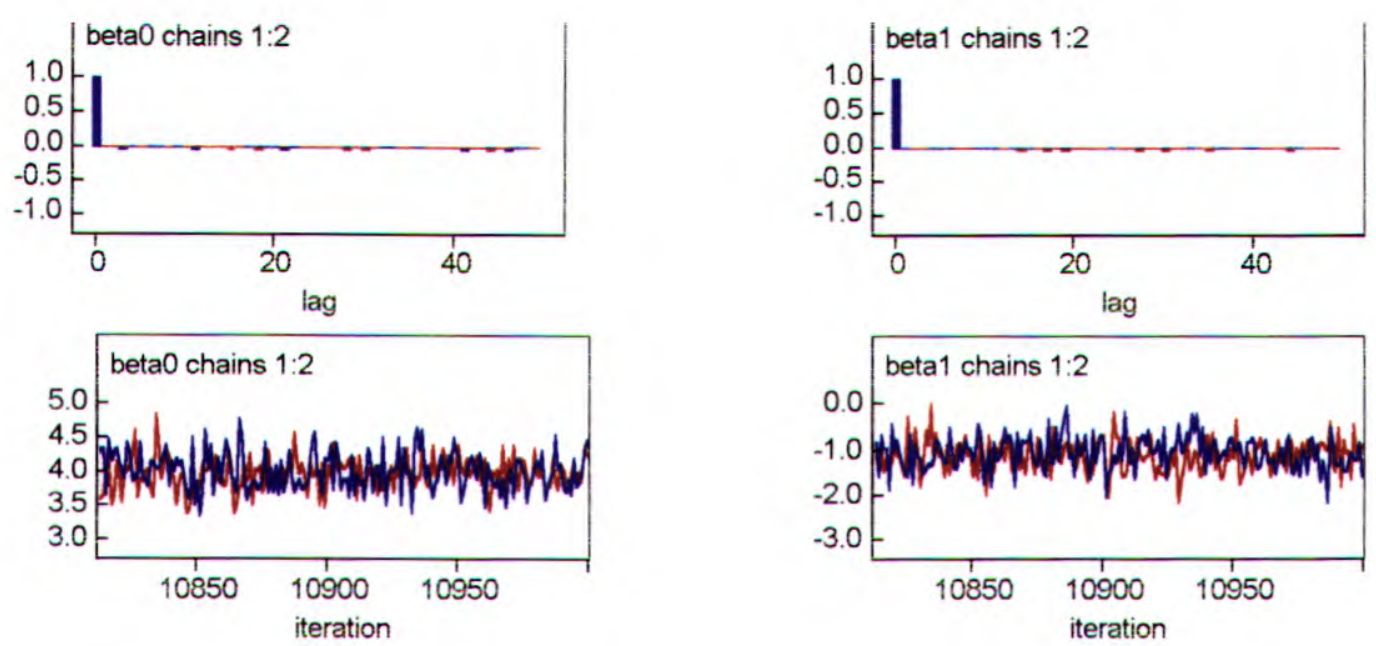

Figura 3.1: Gráficos a posteriori dos parâmetros para o modelo exponencial.

Usamos 2 cadeias com 11.000 amostras cada e descartamos as primeiras 1000 amostras("burn-in sampling "), pelos gráficos de auto- correlação observamos que só existe correlação em números consecutivos, assim, na amostra final, selecionamos os números de 2 em 2 e totalizamos um total de 5000 amostras em cada cadeia.

Pelas figuras notamos um comportamento bom para elas e por isso consideramos que as estimativas Bayesianas estão boas.

\subsubsection{Modelo de Regressão Weibull}

Agora assumimos o modelo de regressão Weibull ( seção 2.2.2).

As estimativas clássicas e Bayesianas são dadas estão na Tabela 3.4. Para a análise Bayesiana usamos as distribuições a priori dadas em 2.2.2.2., com valores dos hiperparâmetros dados por: $a=10, b=10, c_{\mathrm{j}}=0$ e $d_{j}=1000$.

Tabela 3.4 Estimativas para o modelo de regressão Weibull

\begin{tabular}{c|c|c|c|c}
\hline Parâmetro & EMV & $\begin{array}{c}\text { Intervalo de } \\
\text { Confiança } \\
(95 \%)\end{array}$ & $\begin{array}{c}\text { Média a } \\
\text { posteriori }\end{array}$ & $\begin{array}{c}\text { Intervalo de } \\
\text { Credibilidade } \\
(95 \%)\end{array}$ \\
\hline$\beta_{0}$ & 3,9426 & $(3,451 ; 4,433)$ & 3,957 & $(3,382 ; 4,584)$ \\
\hline$\beta_{1}$ & $-1,0983$ & $(-1,916 ;-0,279)$ & $-1,132$ & $(-2,221 ;-0,184)$ \\
\hline$\sigma^{*}$ & 0,9785 & $(0,591 ; 1,365)$ & 1,1200 & $(0,625 ; 1,614)$ \\
\hline
\end{tabular}


Tabela 3.5: Vaiores obtidos para $\sqrt{R}$ no critério de convergência de Gelman e Rubin para os parâmetros.

\begin{tabular}{c|c}
\hline & $\sqrt{R}$ \\
\hline$\beta_{0}$ & 1,000 \\
\hline$\beta_{1}$ & 1,000 \\
\hline$\sigma$ & 1,000 \\
\hline
\end{tabular}

Na Tabela 3.5, temos os valores dos coeficientes de Gelman e Rubin que indicam convergência das cadeias.
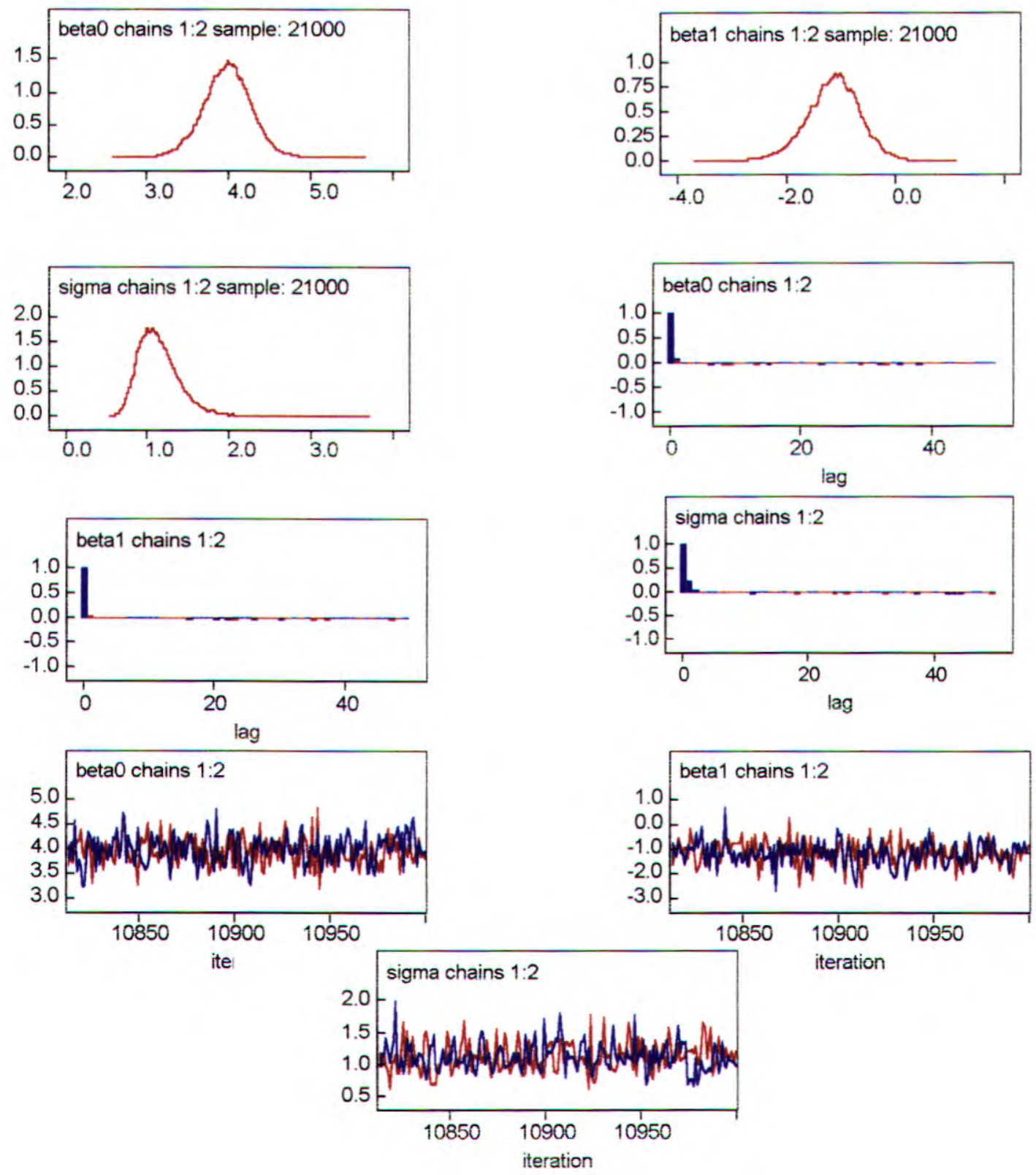

Figura 3.2: Gráficos a posteriori dos parâmetros para o modelo Weibull. 
Na Figura 3.2 temos os gráficos das distribuições a posteriori marginais como no modelo anterior.

Usamos 2 cadeias com 11.000 amostras cada e descartamos as primeiras 1000 amostras("burn-in sampling "), pelos gráficos de auto- correlação observamos que só existe correlaçăo em números consecutivos para os parâmetros $\beta_{0}$ e $\beta_{1}$. Para $\sigma$ temos uma correlação até o segundo número, assim, selecionamos de 3 em 3 e ficamos com uma amostra final igual a 3333 em cada cadeia.

\subsubsection{Modelo de Regressão Logístico}

Nesta seção assumimos o modelo de regressão log-logístico na seção (2.2.3).

As estimativas clássicas e Bayesianas são dadas na Tabela 3.6. Para uma análise Bayesiana consideramos as mesmas distribuições a priori utilizadas no modelo anterior.

Tabela 3.6 Estimativas para o modelo de regressão Log-Logístico

\begin{tabular}{c|c|c|c|c}
\hline Parâmetro & EMV & $\begin{array}{c}\text { Intervalo de } \\
\text { Confiança } \\
(95 \%)\end{array}$ & $\begin{array}{c}\text { Média a } \\
\text { posteriori }\end{array}$ & $\begin{array}{c}\text { Intervalo de } \\
\text { Credibilidade } \\
(95 \%)\end{array}$ \\
\hline$\beta_{0}$ & 3,345 & $(2,755 ; 3,934)$ & 3,354 & $(2,698 ; 4,015)$ \\
\hline$\beta_{1}$ & $-1,935$ & $(-2,938 ;-0,931)$ & $-1,927$ & $(-3,042 ;-0,804)$ \\
\hline$\sigma^{*}$ & 0,680 & $(0,417 ; 0,942)$ & 0,759 & $(0,503 ; 1,151)$ \\
\hline
\end{tabular}

Tabela 3.7: Valores obtidos para $\sqrt{ } R$ no critério de convergência de Gelman e Rubin para os parâmetros.

\begin{tabular}{c|c}
\hline & $\sqrt{R}$ \\
\hline$\beta_{0}$ & 1,000 \\
\hline$\beta_{1}$ & 1,000 \\
\hline$\sigma$ & 1,000 \\
\hline
\end{tabular}


Novamente, notamos pela Tabela 3.7 que houve a convergência de todos os parâmetros.

Na Figura 3.3 temos os gráficos das distribuições a posteriori marginais .
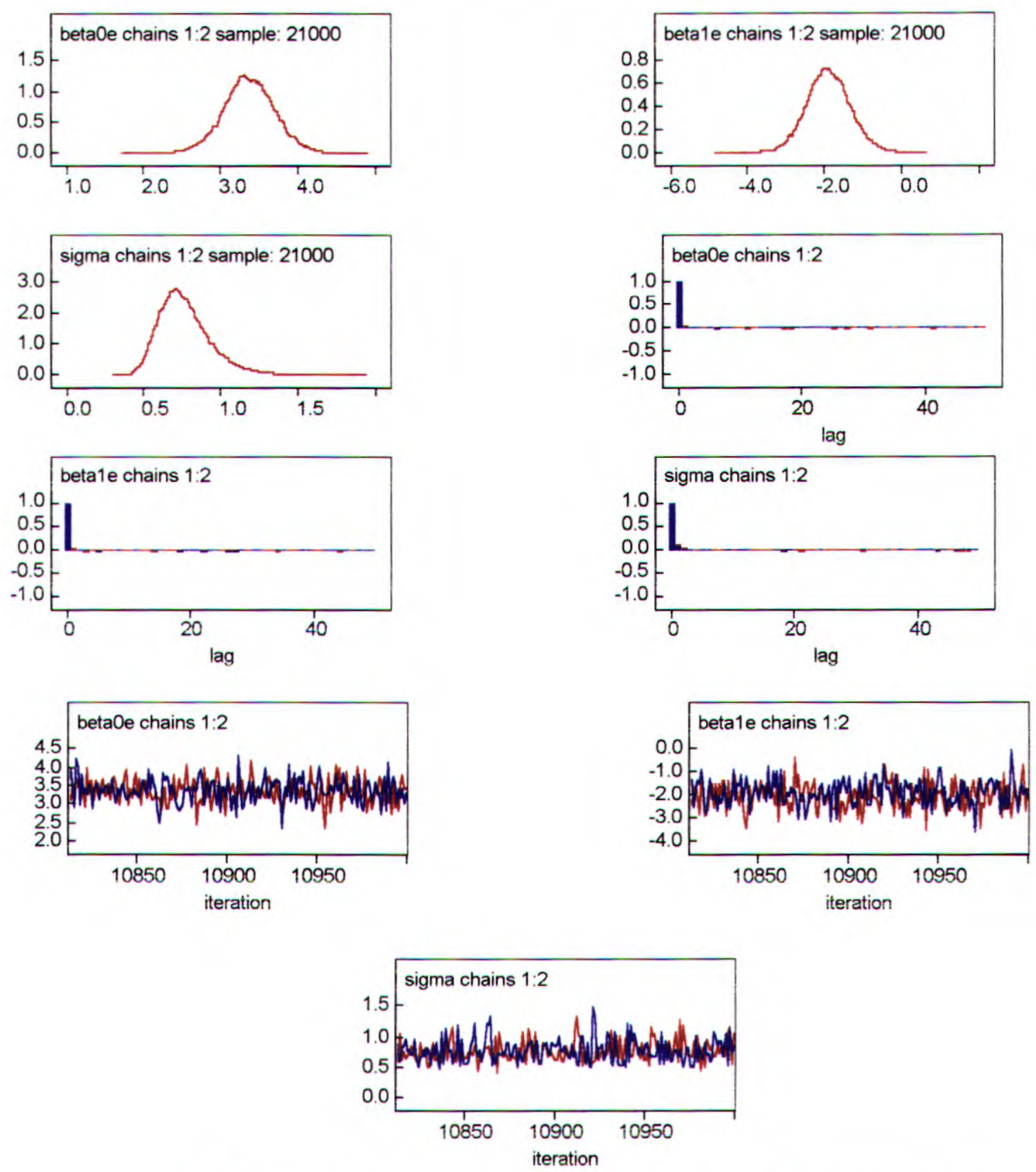

Figura 3.3: Gráficos a posteriori dos parâmetros para o modelo Log-Logístico.

As amostras finais para este modelo foram retiradas da mesma maneira do modelo Weibull, já que os parâmetros tiveram um comportamento muito parecido em ambos os modelos. 


\subsubsection{Modelo de Regressão Log- Normal}

Aqui, assumimos o modelo visto na seção 2.2.4. Novamente lembramos que as priori e qualquer outra informação mais precisa como a função de verossimilhança podem ser encontradas na seção citada acima.

As estimativas clássicas e Bayesianas são dadas na Tabela 3.8. Neste caso também consideramos as mesmas distribuições a priori consideradas previamente para os outros modelos.

Tabela 3.8 Estimativas para o modelo de regressão Log-normal

\begin{tabular}{c|c|c|c|c}
\hline Parâmetro & EMV & $\begin{array}{c}\text { Intervalo de } \\
\text { Confiança } \\
(95 \%)\end{array}$ & $\begin{array}{c}\text { Média a } \\
\text { Posteriori }\end{array}$ & $\begin{array}{c}\text { Intervalo de } \\
\text { Credibilidade } \\
(95 \%)\end{array}$ \\
\hline$\beta_{0}$ & 3,361 & $(2,811 ; 3,908)$ & 3,355 & $(2,722 ; 3,992)$ \\
\hline$\beta_{1}$ & $-1,886$ & $(-2,789 ;-0,982)$ & $-1,887$ & $(-2,928 ;-0,832)$ \\
\hline$\sigma^{*}$ & 1,155 & $(0,767 ; 1,543)$ & 1,751 & $(0,822 ; 3,623)$ \\
\hline
\end{tabular}

Tabela 3.9: Valores obtidos para $\sqrt{R}$ no critério de convergência de Gelman e Rubin para os parâmetros.

\begin{tabular}{c|c}
\hline & $\sqrt{R}$ \\
\hline$\beta_{0}$ & 1,000 \\
\hline$\beta_{1}$ & 1,000 \\
\hline$\sigma$ & 1,000 \\
\hline
\end{tabular}

Observamos que o critério de Gelman Rubin foi satisfeito.

Na Figura 3.4 temos os gráficos das distribuições a posteriori marginais .
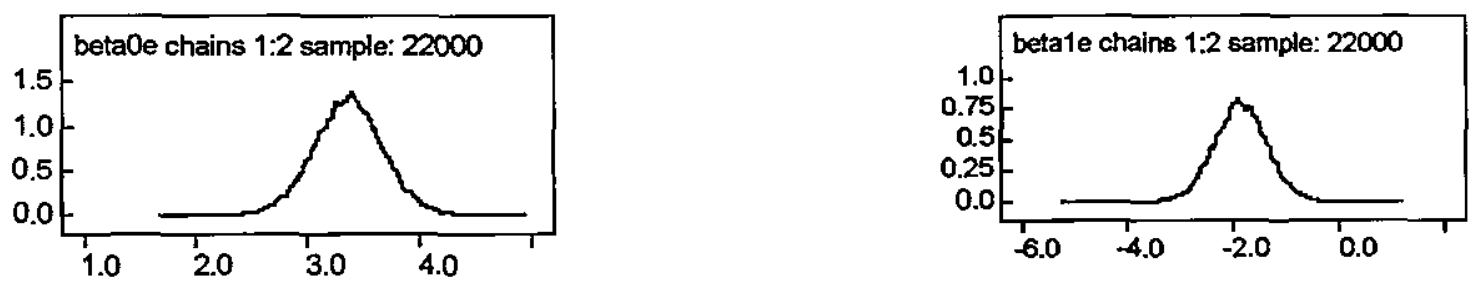

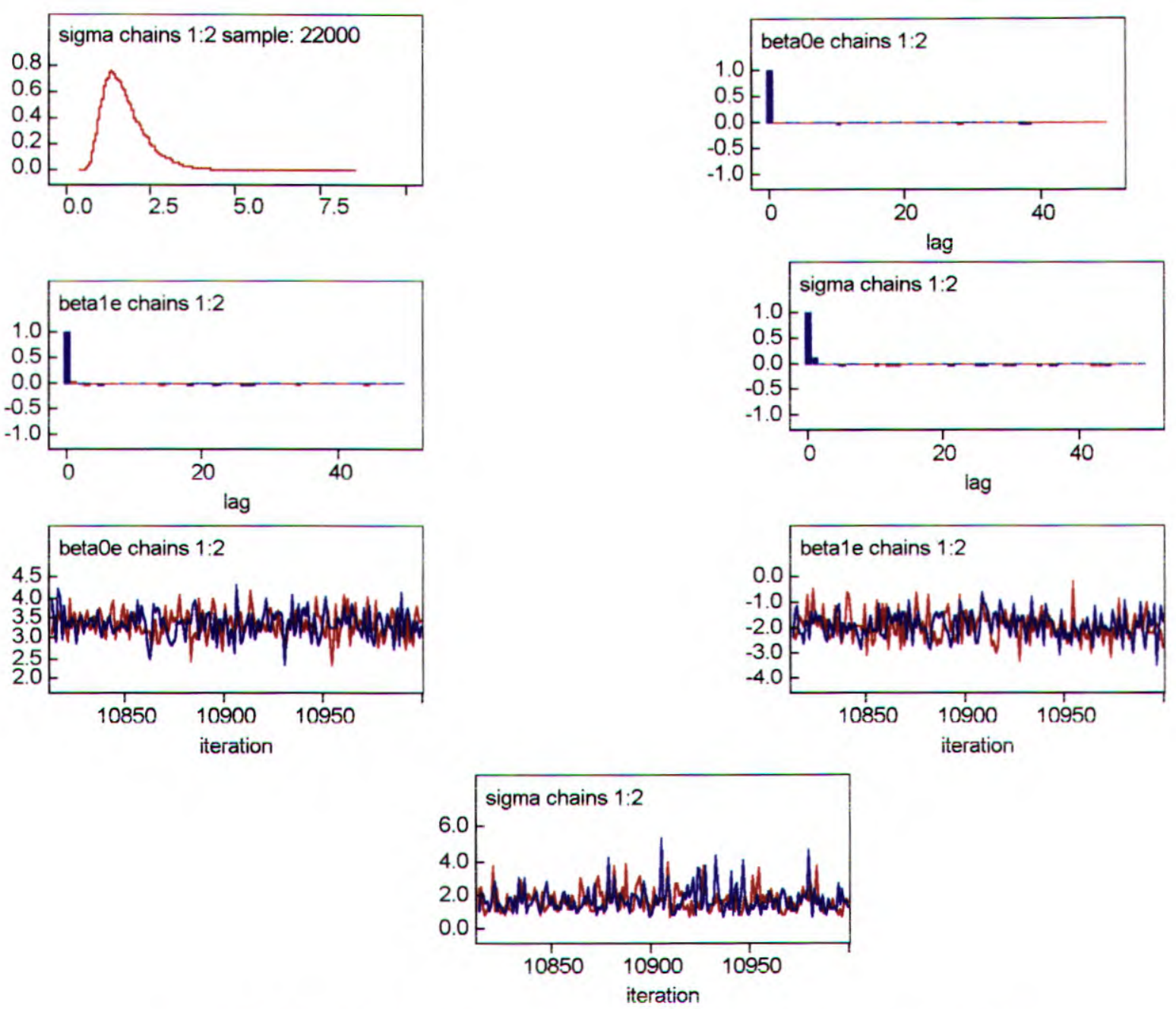

Figura 3.4: Gráficos a posteriori dos parâmetros para o modelo Log-Normal.

Novamente temos a amostra final igual a feita no modelo Weibull, ou seja, um total de 3333 amostras para cada parâmetro em cada cadeia.

\subsection{Segundo Conjunto de Dados}

Na Tabela 3.10 temos os números de ciclos até falha de um grupo de 60 aparelhos elétricos em um teste de vida (Lawless, 1982). Os tempos de falha foram ordenados por conveniência. Na Figura 3.5, temos o histograma das observações no qual podemos notar uma bimodalidade, o que pode sugerir o uso de uma mistura de 2 distribuições para analisar os dados. 
Tabela 3.10: Tempos de falhas de 60 aparelhos elétricos

\section{Tempos de falhas}

\begin{tabular}{rrrrrrrrrrrr}
\hline 14 & 34 & 59 & 61 & 69 & 80 & 123 & 142 & 165 & 210 & 381 & 464 \\
479 & 556 & 574 & 839 & 917 & 969 & 991 & 1064 & 1088 & 1091 & 1174 & 1270 \\
1275 & 1355 & 1397 & 1477 & 1578 & 1649 & 1702 & 1893 & 1932 & 2001 & 2161 & 2292 \\
2326 & 2337 & 2628 & 2785 & 2811 & 2886 & 2993 & 3122 & 3248 & 3715 & 3790 & 3857 \\
3912 & 4100 & 4106 & 4116 & 4315 & 4510 & 4584 & 5267 & 5299 & 5583 & 6065 & 9701 \\
\hline
\end{tabular}

Histograma dos Tempos de Falha (Materiais Elétricos)

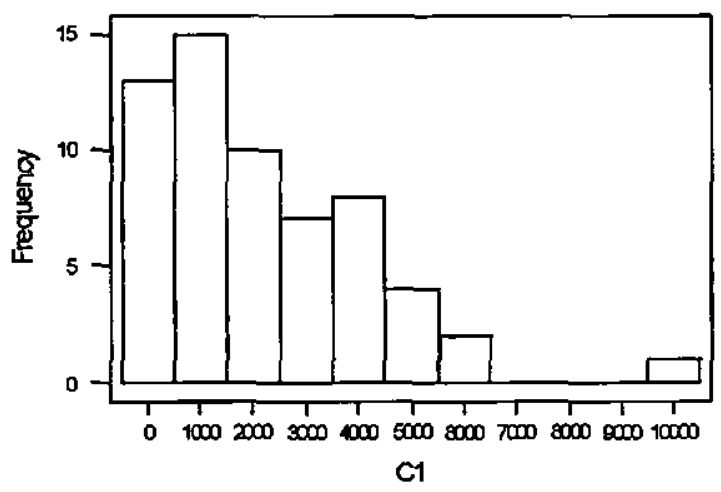

Figura 3.5: Histograma dos dados da Tabela 3.10.

Esse segundo conjunto de dados não possui nenhuma covariável mas é interessante já que ele sugere uma mistura de distribuições que veremos no próximo capitulo. Nesta etapa, apresentaremos alguns modelos mais simples como Exponencial, Weibull entre outros, para analisar os dados da Tabela 3.10.

Aqui, também não temos dados censurados e é importante observar agora que $\mathbf{\beta}$ é igual a $\beta_{0}$ somente. Para uma análise Bayesinana consideramos a distribuição a priori dada em (2.15) com os seguintes valores para os hiperparâmetros: $a=0, b=1000$.

\subsubsection{Modelo de Regressấo Exponencial}


Assumir um modelo de regressão log-linear exponencial (ver seção 2.9).

Assim, temos na Tabela 3.11 as estimativas clássicas e Bayesianas para este modelo,

Tabela 3.11: Estimativas clássicas e Bayesinas para 0 modelo exponencial

\begin{tabular}{c|c|c|c|c}
\hline Parâmetro & EMV & $\begin{array}{c}\text { Intervalo de } \\
\text { Confiança } \\
(95 \%)\end{array}$ & $\begin{array}{c}\text { Média a } \\
\text { Posteriori }\end{array}$ & $\begin{array}{c}\text { Intervalo de } \\
\text { Credibilidade } \\
(95 \%)\end{array}$ \\
\hline$\beta_{0}$ & 7,693 & $(7,440 ; 7,945)$ & 7,698 & $(7,453 ; 7,959)$ \\
\hline$\sigma^{*}=1$ & & - & & - \\
\hline
\end{tabular}

Na Tabela 3.12, temos o valor que indica convergência das cadeias usando o critério de Gelman e Rubin.

Tabela 3.12: Valores obtidos para $\sqrt{R}$ no critério de convergência de Geiman e Rubin para o parâmetro.

\begin{tabular}{c|c}
\hline & $\sqrt{R}$ \\
\hline$\beta_{0}$ & 1,000 \\
\hline
\end{tabular}

Na Figura 3.6 temos os gráficos das distribuições a posteriori marginais do parâmetro de interesse.

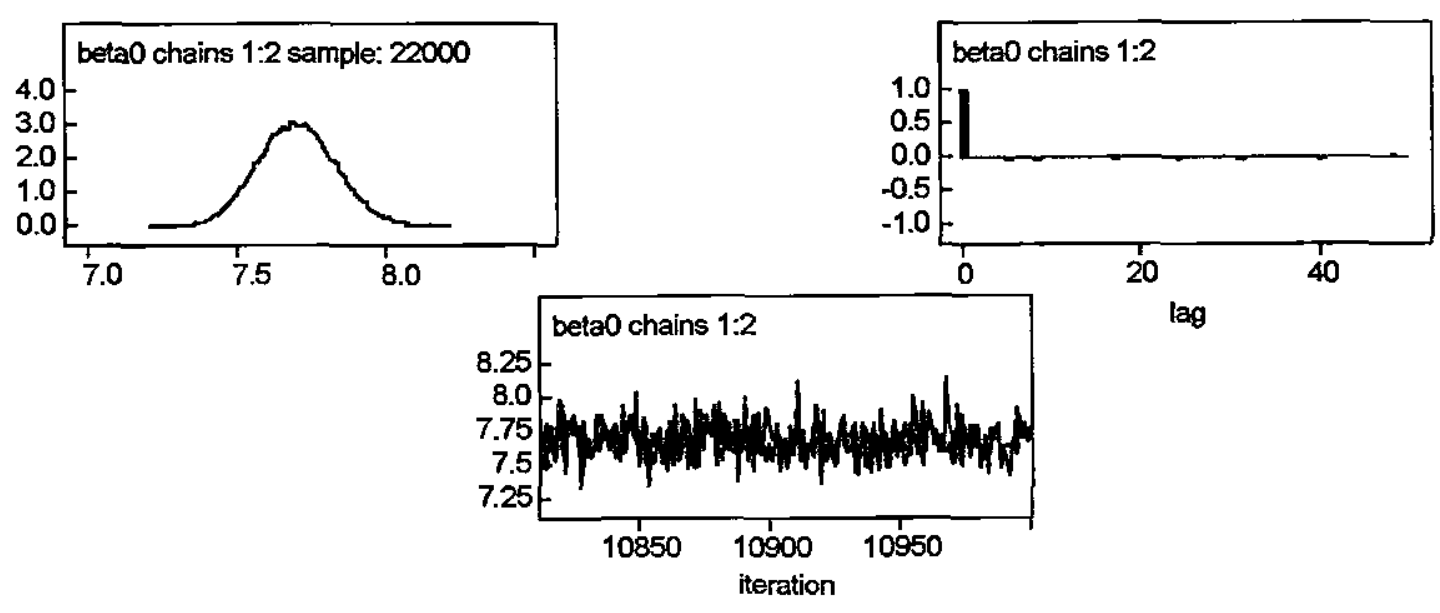

Figura 3.6: Gráficos a posteriori dos parâmetros para o modelo Exponencial dos dados 2.

Geramos duas cadeias com 11000 amostras cada uma, descartamos as primeiras 1000 como nos dados anteriores e para este caso, 
selecionamos a amostra final de $2 \mathrm{em} 2$ como sugerido na figura acima. Então, resultamos em uma amostra final de tamanho 10000 (5000 em cada cadeia).

\subsubsection{Modelo de Regressão Weibull}

Agora vamos ajustar um modelo Weibull para os dados.

Assim, temos na Tabela 3.13 abaixo as estimativas clássicas e Bayesianas para este modelo, onde consideramos as distribuiçőes a priori dadas na seção 2.2.2.2 com os seguintes valores para os hiperparâmetros: $a=10, b=10, c_{\mathrm{j}}=0$ e $d_{j}=1000$.

Tabela 3.13: Estimativas clássicas e Bayesianas para o modelo Weibull

\begin{tabular}{c|c|c|c|c}
\hline Parâmetro & EMV & $\begin{array}{c}\text { Intervalo de } \\
\text { Confiança } \\
(95 \%)\end{array}$ & $\begin{array}{c}\text { Média a } \\
\text { posteriori }\end{array}$ & $\begin{array}{c}\text { Intervalo de } \\
\text { Credibilidade } \\
(95 \%)\end{array}$ \\
\hline$\beta_{0}$ & 7,695 & $(7,430 ; 7,955)$ & 7,697 & $(7,414 ; 7,961)$ \\
\hline$\sigma$ & 0,999 & $(0,791 ; 1,206)$ & 1,013 & $(0,821 ; 1,125)$ \\
\hline
\end{tabular}

É importante notar que o valor de $\sigma$ é próximo de 1 , e tanto o intervalo clássico como o Bayesiano cobrem esse valor. Assim, temos uma forte evidência que o modelo exponencial se aplica melhor que o Weibull neste caso já que sempre preferimos modelos mais simples aos mais complexos.

$\mathrm{Na}$ Tabela 3.14, temos os valores que indicam convergência das cadeias usando o critério de Gelman e Rubin.

Tabela 3.14: Valores obtidos para $\sqrt{R}$ no critério de convergência de Gelman e Rubin para os parâmetros.

\begin{tabular}{c|c}
\hline & $\sqrt{R}$ \\
\hline$\beta_{0}$ & 1,000 \\
\hline$\sigma$ & 1,000 \\
\hline
\end{tabular}


Na Figura 3.7 temos os gráficos das distribuições a posteriori marginais do parâmetro de interesse.
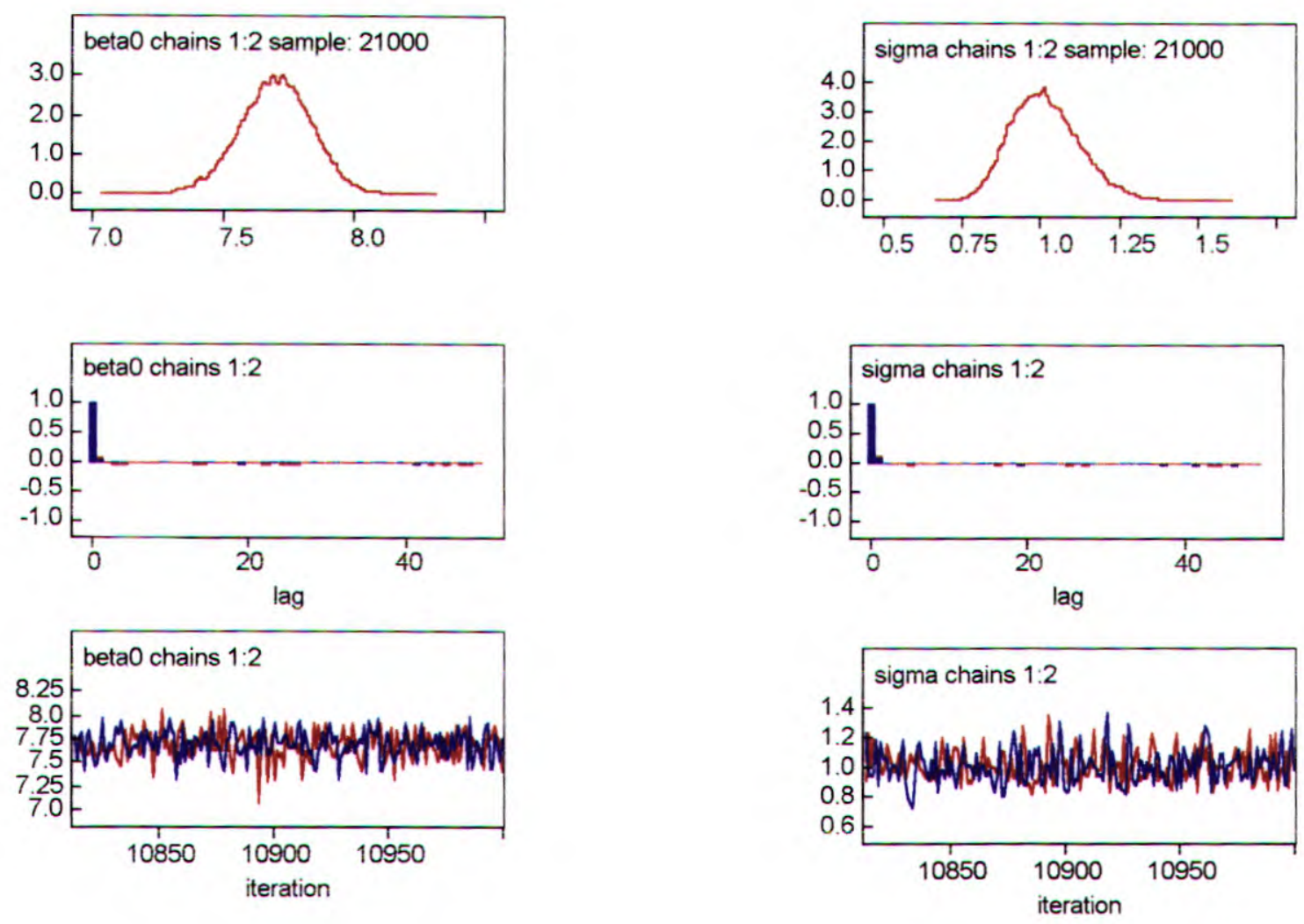

Figura 3.7: Gráficos a posteriori dos parâmetros para o modelo Weibull dos dados 2.

Para os dados 2 , observamos que o parâmetro $\sigma$ se comportou de maneira parecida com o parâmetro de regressão. Assim, selecionamos amostras de 2 em 2, o que totalizou uma amostra final de 10000 para cada um dos parâmetros. Além disso, a Figura 3.7 nos mostra a convergência das cadeias para o mesmo ponto.

\subsubsection{Modelo de Regressão Log - Logístico}

O modelo aqui utilizado foi baseado em 2.2.3.

$\mathrm{Na}$ Tabela 3.15 abaixo, temos as estimativas clássicas e Bayesianas para este modelo. Consideramos as mesmas distribuições a priori do modelo anterior. 
Tabela 3.15: Estimativas clássicas e Bayesianas para o modelo Weibull

\begin{tabular}{c|c|c|c|c}
\hline Parâmetro & EMV & $\begin{array}{c}\text { Intervalo de } \\
\text { Confiança } \\
(95 \%)\end{array}$ & $\begin{array}{c}\text { Média a } \\
\text { posteriori }\end{array}$ & $\begin{array}{c}\text { Intervalo de } \\
\text { Credibilidade } \\
(95 \%)\end{array}$ \\
\hline \hline$\beta_{0}$ & 7,276 & $(6,938 ; 7,613)$ & 7,271 & $(6,915 ; 7,615)$ \\
\hline$\sigma$ & 0,773 & $(0,606 ; 0,939)$ & 0,792 & $(0,635 ; 0,987)$ \\
\hline
\end{tabular}

Para o modelo logístico temos que as estimativas foram muito próximas nos casos clássico e Bayesino. Novamente o WinBugs foi utilizado para o caso Bayesino e por isso essas estimativas foram conseguidas de forma bem simples, sem nenhum problema computacional.

$\mathrm{Na}$ Tabela 3.16 , temos os valores que indicam convergência das cadeias usando o critério de Gelman e Rubin.

Tabela 3.16: Valores obtidos para $\sqrt{R}$ no critério de convergência de Geiman e Rubin para os parâmetros.

\begin{tabular}{c|c}
\hline & $\sqrt{R}$ \\
\hline$\beta_{0}$ & 1,000 \\
\hline$\sigma$ & 1,000 \\
\hline \hline
\end{tabular}

$\mathrm{Na}$ Figura 3.8 temos os gráficos das distribuições a posteriori marginais do parâmetro de interesse.
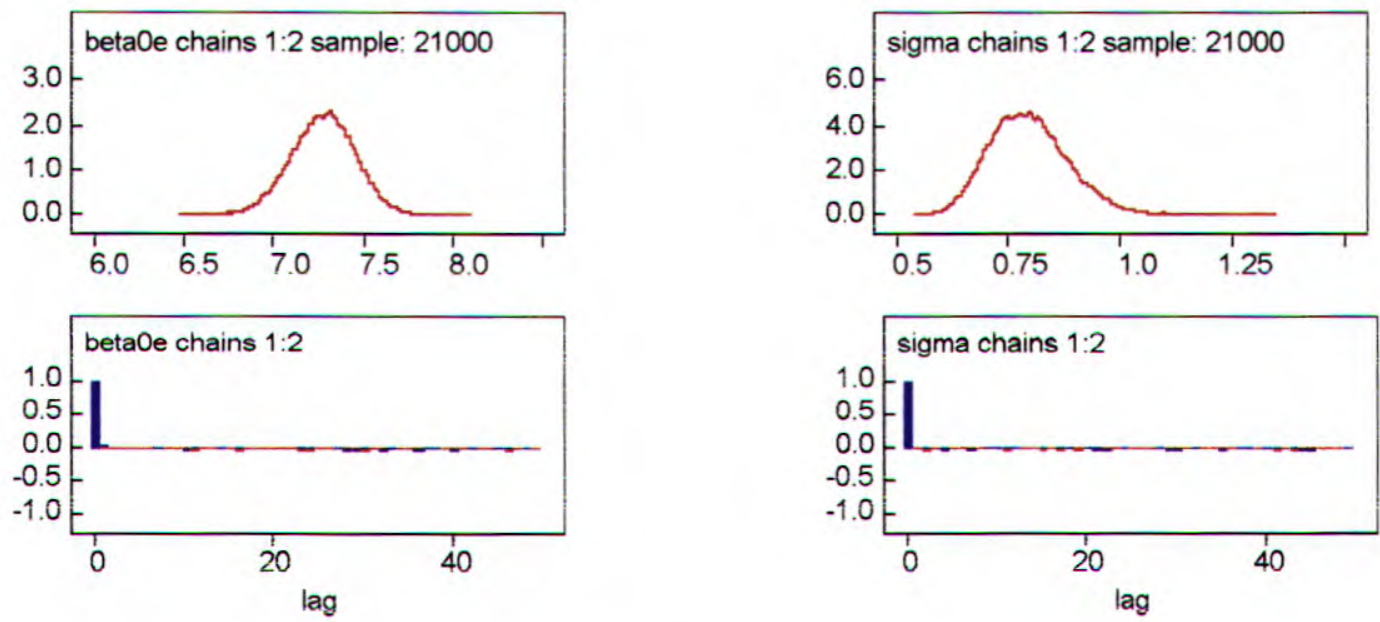

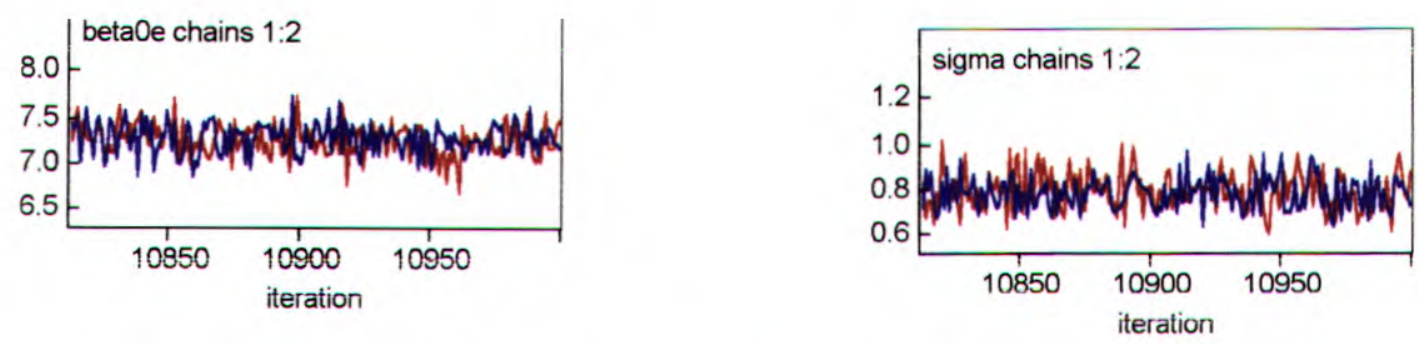

Figura 3.8: Gráficos a posteriori dos parâmetros para o modelo Logístico dos dados 2.

Como no caso Weibull, os parâmetros se comportaram de maneira semelhante no que se refere a auto-correlação. Assim, selecionamos uma amostra de 2 em 2 . Também aqui podemos observar a convergência das cadeias.

\subsubsection{Modelo de Regressão Log - Normal}

Para finalizar a parte feita no software WinBugs, assumir o modelo lognormal para o segundo conjunto de dados da Tabela 3.10. Novamente utilizamos as mesmas distribuições a priori dadas no modelo Weibull.

A Tabela 3.17 abaixo mostra as estimativas clássicas e Bayesianas para este modelo,

Tabela 3.17: Estimativas clássicas e Bayesianas para o modelo Lognormal.

\begin{tabular}{c|c|c|c|c}
\hline Parâmetro & EMV & $\begin{array}{c}\text { Intervalo de } \\
\text { Confiança } \\
(95 \%)\end{array}$ & $\begin{array}{c}\text { Média a } \\
\text { Posteriori }\end{array}$ & $\begin{array}{c}\text { Intervalo de } \\
\text { Credibilidade } \\
(95 \%)\end{array}$ \\
\hline$\beta_{0}$ & 7,067 & $(6,704 ; 7,429)$ & 7,065 & $(6,700 ; 7,432)$ \\
\hline$\sigma$ & 1,439 & $(1,182 ; 1,695)$ & 2,107 & $(1,470 ; 3,007)$ \\
\hline
\end{tabular}

Para o modelo log-normal notamos pela primeira vez uma diferença entre as estimativas clássica e Bayesiana para um dos parâmetros $(\sigma)$. A estimativa Bayesiana está fora do intervalo de confiança clássico. No entanto, como novamente temos uma amostra pequena pode ser que a estimativa clássica tenha tido algum problema na aproximação assintótica.

$\mathrm{Na}$ Tabela 3.18 , temos os valores que indicam convergência das cadeias usando o critério de Gelman e Rubin. 
Tabela 3.18: Valores obtidos para $\sqrt{ } R$ no critério de convergência de Gelman e Rubin para os parâmetros.

\begin{tabular}{c|c}
\hline & $\sqrt{R}$ \\
\hline$\beta_{0}$ & 1,000 \\
\hline$\sigma$ & 1,000 \\
\hline
\end{tabular}

$\mathrm{Na}$ Figura 3.9 temos os gráficos das distribuições a posteriori marginais do parâmetro de interesse.
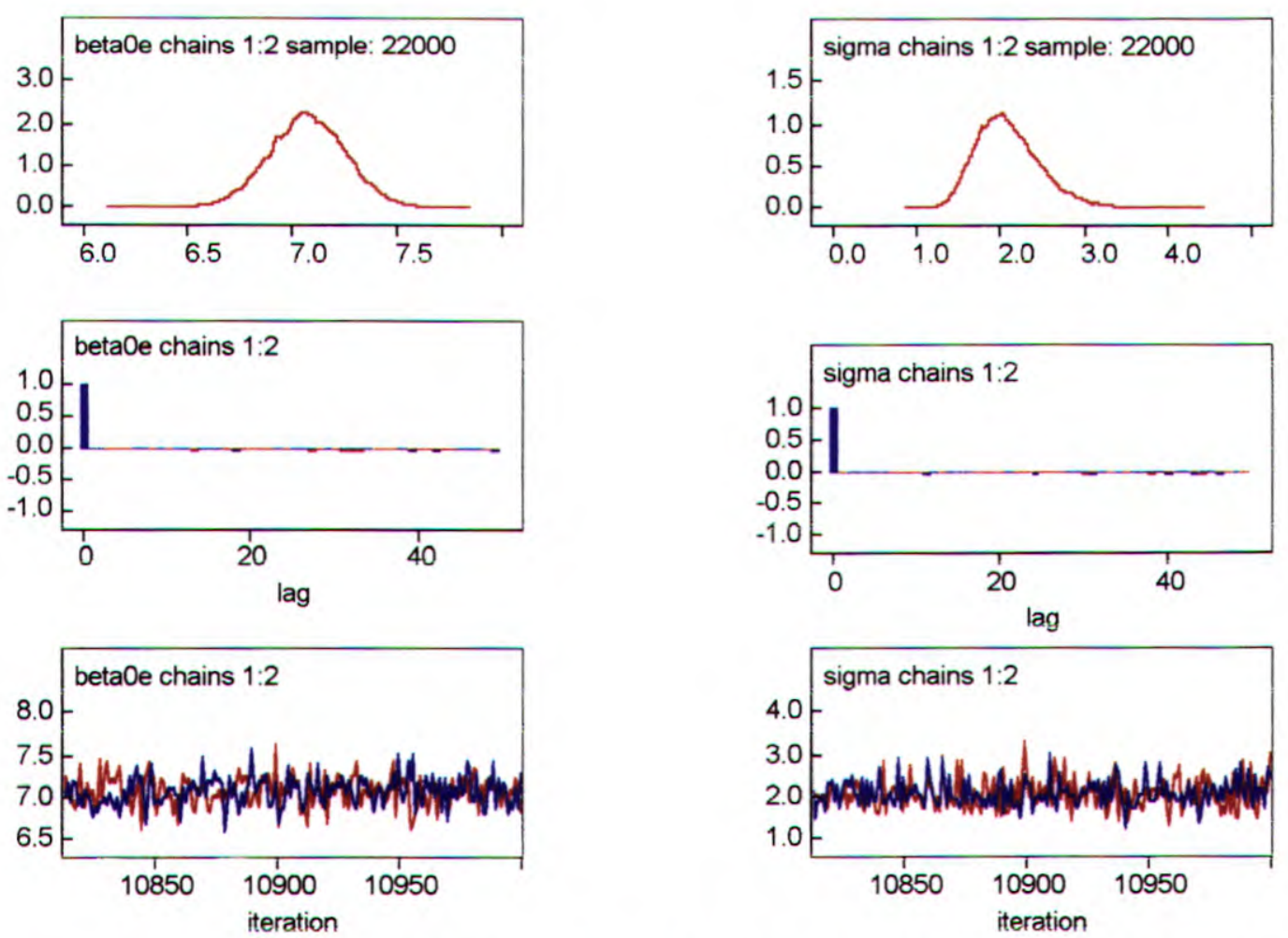

Figura 3.9: Gráficos a posteriori dos parâmetros para o modelo Log- normal dos dados 2.

Neste modelo, vamos observar os gráficos a posteriori com maior atenção. Pelos gráficos apresentados, não temos nenhuma evidência que tenha algum problema com as cadeias do parâmetro sigma. Assim, acreditamos que a estimativa Bayesina esteja boa. Provavelmente uma priori informativa diminuiria o comprimento do intervalo de credibilidade. 


\subsubsection{Modelo de Regressão Gama Generalizada}

Além dos modelos ajustados para os dados da Tabela 3.10, também ajustamos o modelo Gama Generalizada já que é um supermodelo e queríamos testá-lo.

$\mathrm{Na}$ Tabela 3.19 abaixo, temos as estimativas clássicas e Bayesianas para este modelo, considerando as distribuições a priori dadas na seção 2.2.5.2 com os seguintes valores para os hiperparâmetros da priori para $k: a=1, b=1$.

Iabela 5.7y: Estumativas clássicas e Bayesinas para o modelo Gama Generallzado.

\begin{tabular}{c|c|c|c|c}
\hline Parâmetro & EMV & $\begin{array}{c}\text { Intervalo de } \\
\text { Confiança } \\
(95 \%)\end{array}$ & $\begin{array}{c}\text { Média a } \\
\text { posteriori }\end{array}$ & $\begin{array}{c}\text { Intervalo de } \\
\text { Credibilidade } \\
(95 \%)\end{array}$ \\
\hline$\beta_{0}$ & 8,51 & $(4,805 ; 12,214)$ & 7,762 & $(6,192 ; 8,948)$ \\
\hline$\sigma$ & 2,15 & $(0,68 ; 3,61)$ & 2,164 & $(1,808 ; 2,861)$ \\
\hline $\mathrm{k}$ & 0,33 & $(0,031 ; 0,638)$ & 0,337 & $(0,291 ; 0,460)$ \\
\hline
\end{tabular}

Para este modelo, temos estimativas distintas entre os parâmetros de regressão. No entanto, a estimativa Bayesiana fica bem mais próxima das estimativas obtidas para outros modelos.

Como este modelo é um modelo mais complexo pode ser que a estimativa clássica não tenha boa precisão usando métodos aproximados. Lembramos aqui que as estimativas clássicas foram feitas pelo procedimento nlp do software SAS e assim. Delo método numérico de Newton- Raphson. Um método diferente poderia melhorar essas estimativas mas não focalizamos nossa atencao Duscanco estucar o caso clássico.

$\mathrm{Na}$ Tabela 3.20 , temos os valores que indicam convergência das cadeias usando o critério de Geweke. Utilizamos esse critério pois o programa foi feito no software Ox e nele geramos apenas uma cadeia de cada parâmetro. 
Tabela 3.20: Valores obtidos no critério de convergência de Geweke para os parâmetros.

\begin{tabular}{c|c}
\hline & Geweke \\
\hline$\beta_{0}$ & $-0,080$ \\
\hline$\sigma$ & 0,014 \\
\hline$k$ & 0,023 \\
\hline
\end{tabular}

Utilizando $\circ$ Geweke (ver Anexo) temos que o critério diagnostica convergência se os valores encontrados são em módulo menores que 2. Assim, pela Tabela 3.20 temos a convergência de todos os parâmetros.

Na Figura 3.10 temos os gráficos das distribuições a posteriori marginais do parâmetro de interesse. Esses gráficos são diferentes dos anteriores porque os outros foram feitos no software WinBUGS e este no software S-Plus. O motivo para essa troca de software é que as estimativas para o modelo Gama Generalizada foram feitas no OX e portanto não conseguimos fazer os gráficos como anteriormente.
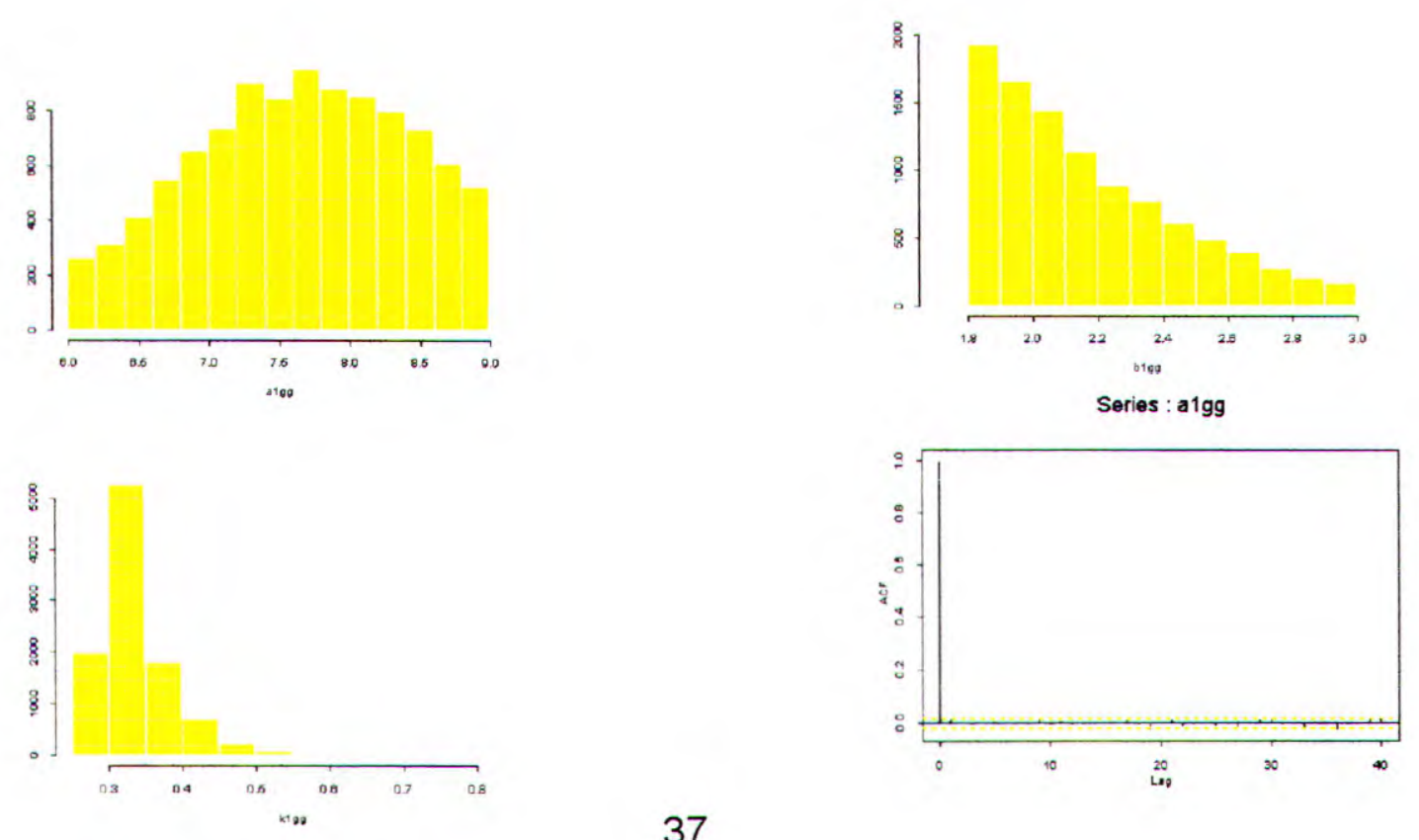

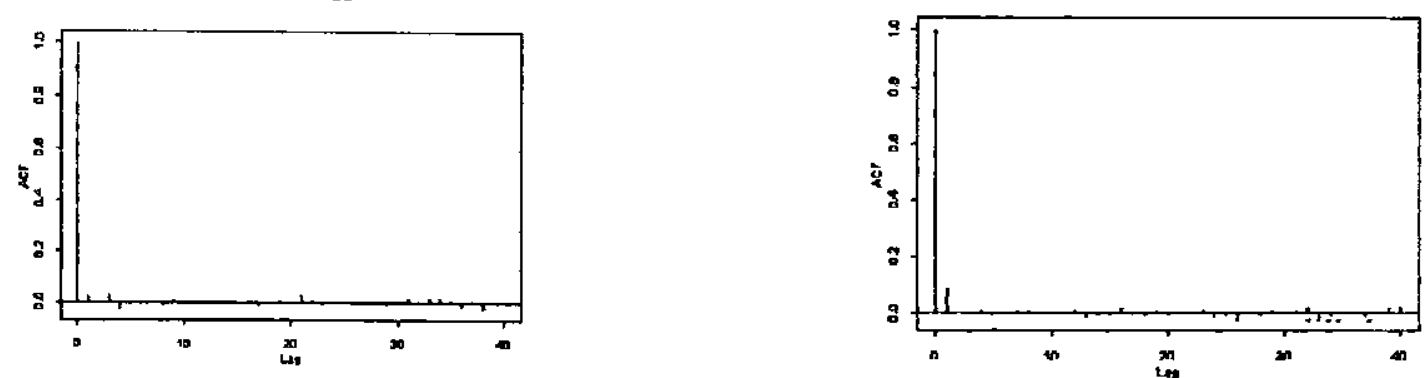

Figura 3.10: Gráficos a posteriori dos parâmetros para o modelo Gama Generalizado dos dados 2.

Para obtermos a amostra final, geramos 11000 amostras para cada parâmetro e descartamos as primeiras 1000 amostras. Depois selecionamos a amostra de $3 \mathrm{em} 3$, resultando numa amostra final de 3333 valores para cada parâmetro.

\subsection{Terceiro Conjunto de Dados}

Na Tabela 3.21 os tempos de fadigas de 20 materiais elétricos. A segunda coluna possui os logaritmos desses valores.

Tabela 3.21: Fadiga de 20 aparelhos elétricos

\begin{tabular}{c|c}
\hline$T$ & $Y=\log (\mathrm{t})$ \\
\hline 233324 & 12,3602 \\
\hline 682811 & 13,4340 \\
\hline 353445 & 12,7755 \\
\hline 208099 & 12,2458 \\
\hline 294337 & 12,5925 \\
\hline 1000000 & 13,8155 \\
\hline 1000000 & 13,8155 \\
\hline 219174 & 12,2976 \\
\hline 119463 & 11,6908 \\
\hline 454405 & 13,0267 \\
\hline 1000000 & 13,8155 \\
\hline 449042 & 13,0149 \\
\hline 228554 & 12,3395 \\
\hline 133039 & 11,7984 \\
\hline 190574 & 12,1578 \\
\hline 1000000 & 13,8155
\end{tabular}


Tabela 3.21(continuação): Fadiga de 20 aparelhos elétricos

\begin{tabular}{l|l}
541840 & 13,2027 \\
\hline 271904 & 12,5132 \\
\hline 166322 & 12,0217 \\
\hline 177115 & 12,0846 \\
\hline
\end{tabular}

Assim como o segundo conjunto de dados da Tabela 310, este também não possui nenhuma covariável mas é interessante pois através dele pudemos estudar uma nova distribuição Birbaum- Saunders. Além disso, alguns modelos de mistura foram ajustados e serão mostrados no Capítulo seguinte.

\subsubsection{Modelo de Regressão Exponencial}

Novamente, o primeiro modelo a ser ajustado é o modelo exponencial.

As estimativas clássicas e Bayesianas são dadas na Tabela 3.22. As distribuiçōes a priori deste modelo assim como os valores dos hiperparâmetros foram os mesmos utilizados no conjunto de dados anterior.

Tabela 3.22: Estimativas clássicas e Bayesinas para o modelo exponencial

\begin{tabular}{c|c|c|c|c}
\hline Parâmetro & EMV & $\begin{array}{c}\text { Intervalo de } \\
\text { Confiança } \\
(95 \%)\end{array}$ & $\begin{array}{c}\text { Média a } \\
\text { Posteriori }\end{array}$ & $\begin{array}{c}\text { Intervalo de } \\
\text { Credibilidade } \\
(95 \%)\end{array}$ \\
\hline$\beta_{0}$ & 12,98 & $(12,548 ; 13,411)$ & 13,00 & $(12,58 ; 13,46)$ \\
\hline$\sigma^{*}=1$ & & - & & - \\
\hline
\end{tabular}

$\mathrm{Na}$ Tabela 3.23 , temos os valores que indicam convergência das cadeias usando o critério de Gelman e Rubin. 
Tabela 3.23: Valores obtidos para $\sqrt{R}$ no critério de convergência de Gelman e Rubin para o parâmetro.

\begin{tabular}{c|c}
\hline & $\sqrt{R}$ \\
\hline$\beta_{0}$ & 1,000 \\
\hline
\end{tabular}

Na Figura 3.11 temos os gráficos das distribuições a posteriori marginais do parâmetro de interesse.
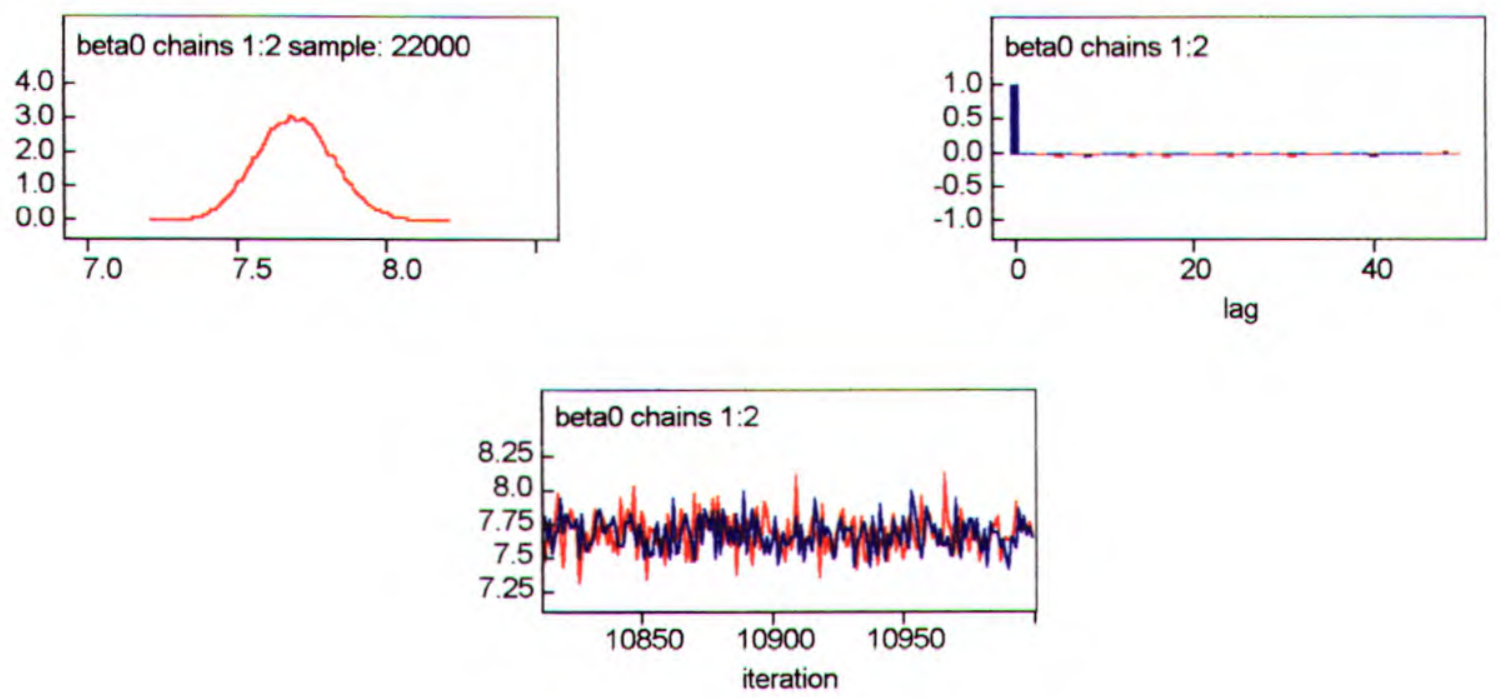

Figura 3.11: Gráficos a posteriori dos parâmetros para o modelo Exponencial dos dados 3.

Neste modelo simples, temos somente um parâmetro a ser estimado e os gráficos a posteriori não indicam nenhum problema. Aqui, selecionamos amostras de 2 em 2, e como o tamanho da cadeia era igual as anteriores ficamos com um total de 10000 valores no total.

\subsubsection{Modelo de Regressão Weibull}

Agora vamos ajustar um modelo Weibull para os dados.

Assim, temos na tabela abaixo as estimativas clássicas e Bayesianas para este modelo, considerando as distribuições a priori com os valores dos hiperparâmetros dados na seção 3.2.2. 
Tabela 3.24: Estimativas clássicas e Bayesianas para o modelo Weibull

\begin{tabular}{c|c|c|c|c}
\hline Parâmetro & EMV & $\begin{array}{c}\text { Intervalo de } \\
\text { Confiança } \\
(95 \%)\end{array}$ & $\begin{array}{c}\text { Média a } \\
\text { posteriori }\end{array}$ & $\begin{array}{c}\text { Intervalo de } \\
\text { Credibilidade } \\
(95 \%)\end{array}$ \\
\hline$\beta_{0}$ & 13,096 & $(12,784 ; 13,407)$ & 13,1 & $(12,76 ; 13,43)$ \\
\hline$\sigma$ & 0,669 & $(0,447 ; 0,890)$ & 0,698 & $(0,496 ; 0,986)$ \\
\hline
\end{tabular}

Para esse conjunto de dados, a estimativa de sigma não está próxima de 1 , então neste caso, o modelo exponencial provavelmente não se ajusta aos dados de forma satisfatória.

As estimativas clássicas e Bayesianas estão bem próximas tanto na estimativa pontual quanto na por intervalo. Como temos dados sem censuras, sem covariáveis e para um modelo simples, não esperávamos nenhuma diferença significativa entre elas.

$\mathrm{Na}$ Tabela 3.25 , temos os valores que indicam convergência das cadeias usando o critério de Gelman e Rubin.

Tabela 3.25: Valores obtidos para $\sqrt{R}$ no critério de convergência de Gelman e Rubin para os parâmetros.

\begin{tabular}{c|c}
\hline & $\sqrt{R}$ \\
\hline$\beta_{0}$ & 1.000 \\
\hline$\sigma$ & 1.000 \\
\hline
\end{tabular}

Na Figura 3.12 temos os gráficos das distribuições a posteriori marginais dos parâmetros de interesse.
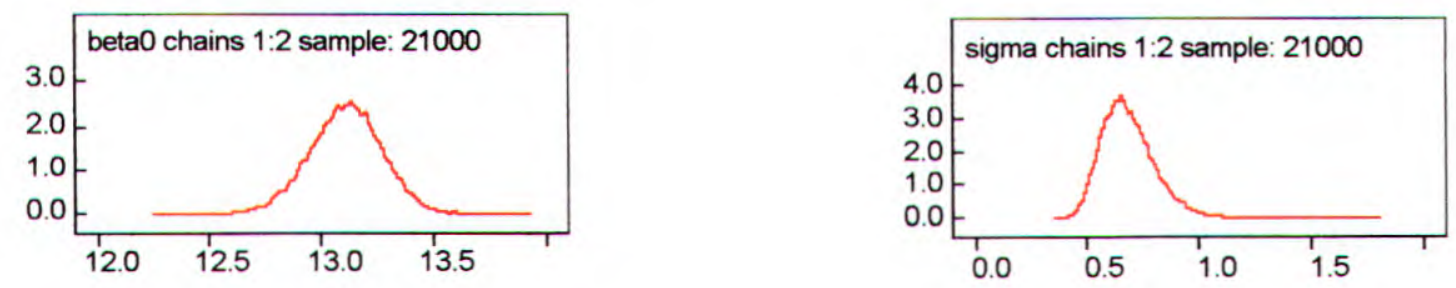

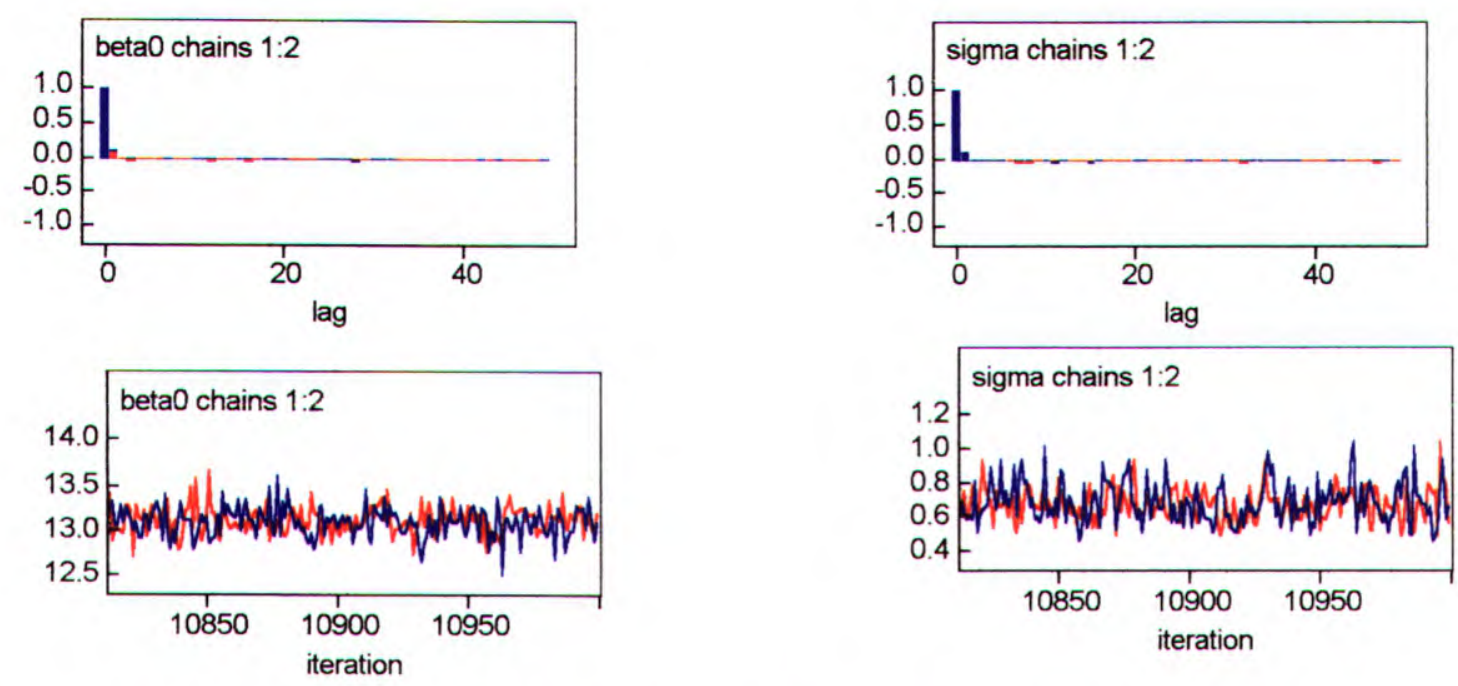

Figura 3.12: Gráficos a posteriori dos parâmetros para o modelo Weibull dos dados 3.

As amostras foram retiradas da mesma maneira que o caso Exponencial.

\subsubsection{Modelo de Regressão Log - Logístico}

O modelo aqui utilizado foi introduzido na seção 2.2.3.

Assim, temos na tabela abaixo as estimativas clássicas e Bayesianas para este modelo, novamente considerando as mesmas distribuições a priori e os mesmos valores dos hiperparâmetros considerados no exemplo anterior.

Tabela 3.26: Estimativas clássicas e Bayesianas para o modelo Weibull

\begin{tabular}{c|c|c|c|c}
\hline Parâmetro & EMV & $\begin{array}{c}\text { Intervalo de } \\
\text { Confiança } \\
(95 \%)\end{array}$ & $\begin{array}{c}\text { Média a } \\
\text { posteriori }\end{array}$ & $\begin{array}{c}\text { Intervalo de } \\
\text { Credibilidade } \\
(95 \%)\end{array}$ \\
\hline$\beta_{0}$ & 12,697 & $(12,369 ; 13,024)$ & 12,7 & $(12,36 ; 13,06)$ \\
\hline$\sigma$ & 0,4178 & $(0,269 ; 0,566)$ & 0,445 & $(0,384 ; 0,9501)$ \\
\hline
\end{tabular}

No modelo logístico, observamos uma diferença um pouco maior para o parâmetro sigma. Novamente o intervalo Bayesiano é bem maior que o clássico.

$\mathrm{Na}$ Tabela 3.27 , temos os valores que indicam convergência das cadeias usando o critério de Gelman e Rubin. 
Tabela 3.27: Valores obtidos para $\sqrt{R}$ no critério de convergência de Gelman e Rubin para os parâmetros.

\begin{tabular}{c|c}
\hline & $\sqrt{R}$ \\
\hline \hline$\beta_{0}$ & 1,000 \\
\hline$\sigma$ & 1,000 \\
\hline
\end{tabular}

$\mathrm{Na}$ figura 3.13 temos os gráficos das distribuições a posteriori marginais do parâmetro de interesse.
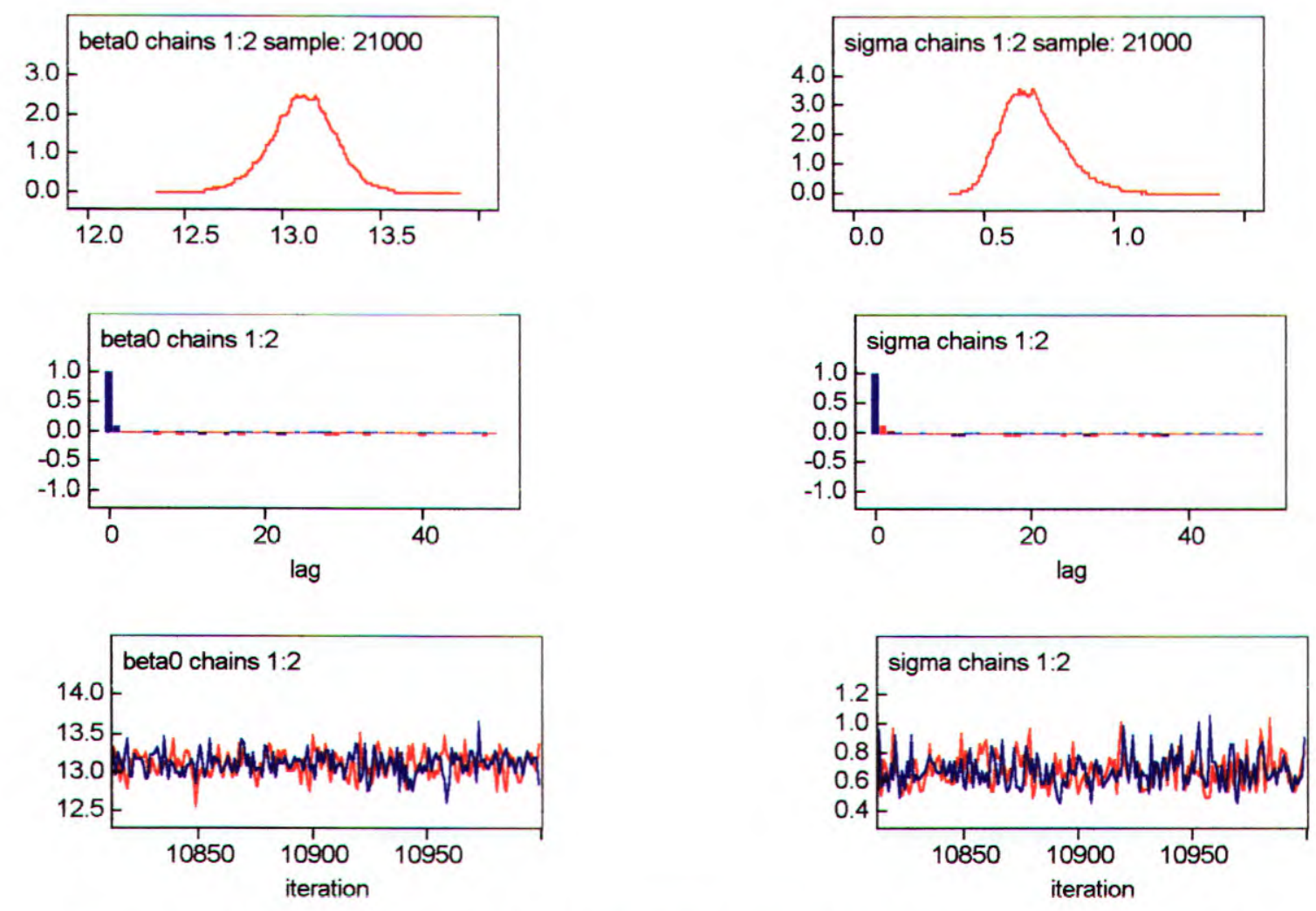

Figura 3.13: Gráficos a posteriori dos parâmetros para o modelo Logístico dos dados 3.

\subsubsection{Modelo de Regressão Log - Normal}

O quarto modelo ajustado para este conjunto de dados é o modelo lognormal. Na Tabela 3.28 , temos as estimativas para este modelo. 
Tabela 3.28: Estimativas clássicas e Bayesianas para o modelo Lognormal.

\begin{tabular}{c|c|c|c|c}
\hline Parâmetro & EMV & $\begin{array}{c}\text { Intervalo de } \\
\text { Confiança } \\
(95 \%)\end{array}$ & $\begin{array}{c}\text { Média a } \\
\text { Posteriori }\end{array}$ & $\begin{array}{c}\text { Intervalo de } \\
\text { Credibilidade } \\
(95 \%)\end{array}$ \\
\hline$\beta_{0}$ & 12,74 & $(12,436 ; 13,043)$ & 12,74 & $(12,43 ; 13,05)$ \\
\hline$\sigma$ & 0,691 & $(0,477 ; 0,904)$ & 0,4927 & $(0,262 ; 0,907)$ \\
\hline
\end{tabular}

Observamos que o modelo log-normal tem um comportamento parecido ao log-logístico, ou seja, a estimativa para o parâmetro $\sigma$ é bem diferente para cada tipo de inferência.

$\mathrm{Na}$ Tabela 3.29 , temos os valores que indicam convergência das cadeias usando o critério de Gelman e Rubin.

Tabela 3.29: Valores obtidos para $\sqrt{R}$ no critério de convergência de Gelman e Rubin para os parâmetros.

\begin{tabular}{c|c}
\hline & $\sqrt{R}$ \\
\hline$\beta_{0}$ & 1,000 \\
\hline$\sigma$ & 1,000 \\
\hline
\end{tabular}

Na Figura 3.14 temos os gráficos das distribuições a posteriori marginais do parâmetro de interesse.
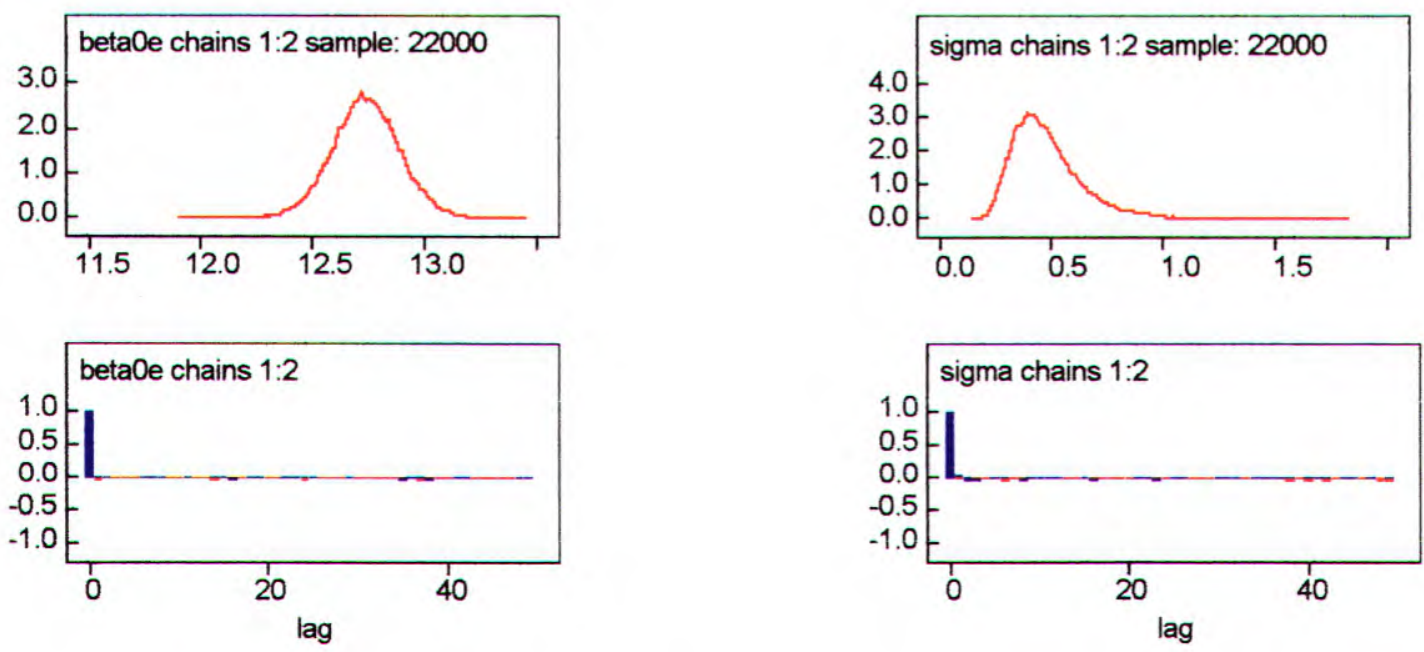

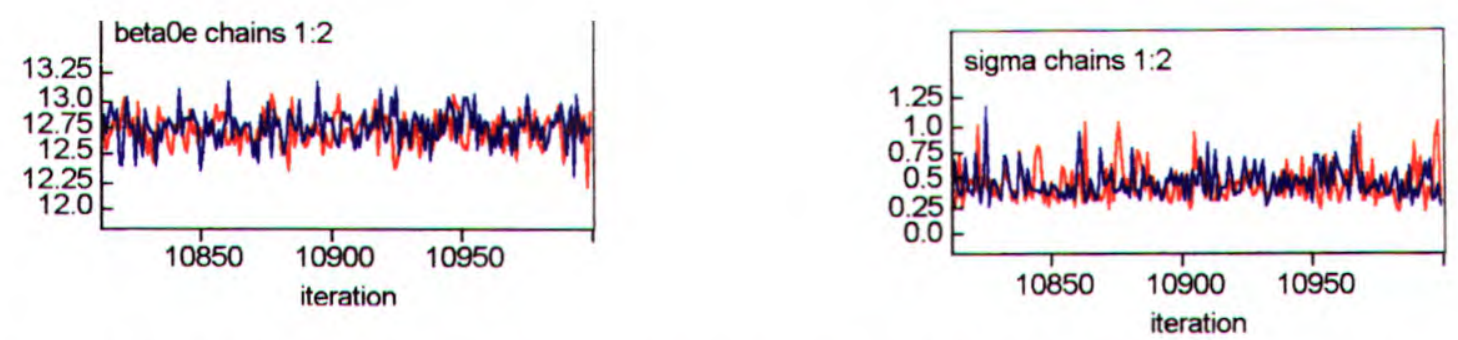

Figura 3.13: Gráficos a posteriori dos parâmetros para o modelo Log- normal dos dados 3.

Os gráficos mostrados na Figura 3.14 são muito parecidos com os anteriores e por isso as conclusões são similares.

\subsubsection{Modelo de Regressão Birbaum Saunders}

Agora apresentaremos um modelo especial para esse tipo de conjunto de dados. Esse modelo só será utilizado nesta seção. As distribuições a priori consideradas neste modelo foram:

$\pi(\alpha) \sim \Gamma(a, b) \quad$; com a e b conhecidos

$\pi(\beta) \sim N\left(c, d^{2}\right) \quad$; com c e d conhecidos

Os valores escolhidos para os hiperparâmetros foram: $a=1, b=1, c=0, d=1000$.

Tabela 3.30: Estimativas clássicas e Bayesianas para o modelo Birbaum-Saunders

\begin{tabular}{c|c|c|c|c}
\hline Parâmetro & EMV & $\begin{array}{c}\text { Intervalo de } \\
\text { Confiança } \\
(95 \%)\end{array}$ & $\begin{array}{c}\text { Média a } \\
\text { posteriori }\end{array}$ & $\begin{array}{c}\text { Intervalo de } \\
\text { Credibilidade } \\
(95 \%)\end{array}$ \\
\hline \hline$\alpha$ & 1,59 & $(1,095 ; 2,084)$ & 1,462 & $(1,024 ; 1,972)$ \\
\hline$\beta$ & 25,6 & $(25,083 ; 26,116)$ & 25,002 & $(23,039 ; 26,926)$ \\
\hline
\end{tabular}

A análise Bayesiana neste caso foi feita utilizando o software OX pois não temos essa distribuição no WinBugs. A comparação deste modelo com os outros será feita no Capítulo 6. 
Tabela 3.31: Valores obtidos no critério de Geweke para os parâmetros.

\begin{tabular}{c|c}
\hline & Geweke \\
\hline$\beta_{0}$ & 0,0583 \\
\hline$\sigma$ & $-0,0069$ \\
\hline
\end{tabular}

Novamente observamos a convergência dos parâmetros já que todos possuem módulo menor que 2 .

Assim como o modelo Gama Generalizada, os gráficos agora apresentados foram feitos no software S-Plus.

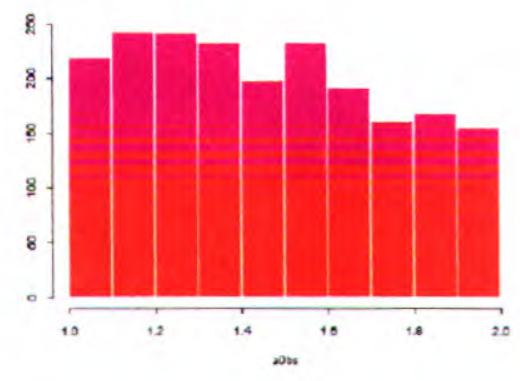

Series : a0bs

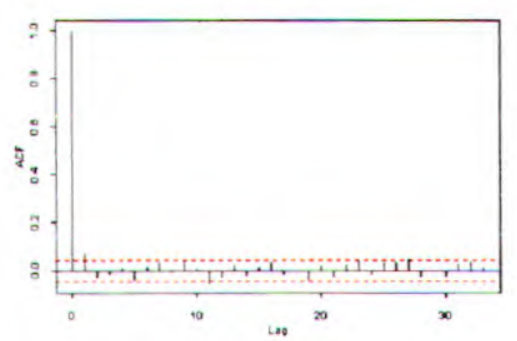

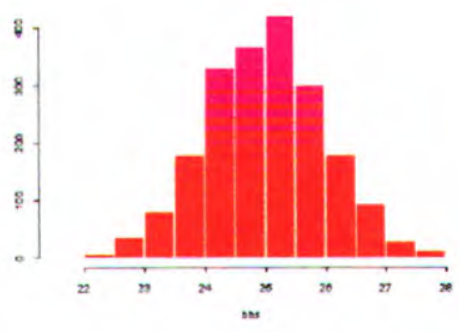

Series : bbs

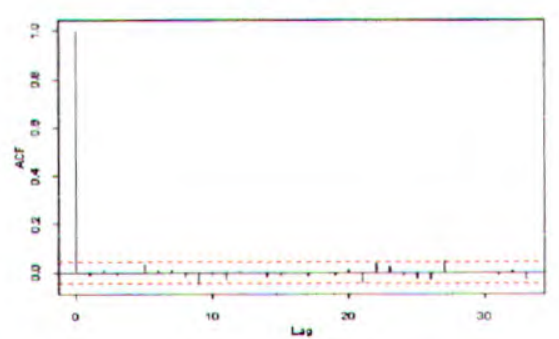

Figura 3.15: Gráficos a posteriori dos parâmetros para o modelo Birbaum-Saunders dados3.

A obtenção de amostras foi feita selecionando valores de 2 em 2. Assim, a amostra final totalizou 5000 valores para cada parâmetro.

\subsection{Quarto Conjunto de Dados}

Para ilustrar modelos com mais de um covariável consideramos os dados de 90 pacientes com câncer na laringe no período de 1970-1978.(Kardaun,1983) 
Neste exemplo, T representa o intervalo, em anos, entre o primeiro tratamento e a ocorrência da morte ou término do estudo $\left(1^{\circ}\right.$ de janeiro de 1983$)$.

Algumas variáveis associadas a estes pacientes são a idade do paciente na data do diagnóstico, o ano do diagnóstico e o estágio da doença. Os quatro estágios da doença são baseados em T.N.M. (tumor primário (T), envolvimento nodal (N), graduação de metastase $(M)$ ).

Temos 4 grupos para estágio do paciente:

1. estágio l: $T_{1} N_{0} M_{0}, c o m 33$ pacientes

2. estágio II: $T_{2} N_{0} M_{0}$, com 17 pacientes

3. estágio III: $T_{3} N_{0} M_{0}$ e $T_{x} N_{1} M_{0}$, com 27 paciente, $x=1,2$ ou 3

4. estágio IV: Todas as outras combinações TNM, exceto TIS com 13 pacientes

Os estágios foram ordenados do caso menos sério para o caso mais sério.

As variáveis dummy foram criadas da seguinte forma:

$$
\begin{aligned}
& Z_{1}= \begin{cases}1 & \text { estágio II } \\
0 & \text { caso contrário }\end{cases} \\
& Z_{2}= \begin{cases}1 & \text { estágio III } \\
0 & \text { caso contrário }\end{cases} \\
& Z_{3}= \begin{cases}1 & \text { estágio IV } \\
0 & \text { caso contrário }\end{cases}
\end{aligned}
$$

A tabela com os dados se encontra no Anexo1.

\subsubsection{Modelo de Regressão Exponencial}

Para analisar esses dados, consideramos o modelo de regressắo (2.6) com $\mathbf{x} \boldsymbol{\beta}=\beta_{0}+\beta_{1} z_{1}+\beta_{2} z_{2}+\beta_{3} z_{3}+\beta_{4} x_{4}$, onde $z_{1}$ denota a primeira variável dummy, $z_{2}$ a segunda e $z_{3}$ a terceira. A covariável $x_{4}$ representa a idade do paciente. 
$\mathrm{Na}$ Tabela 3.32, temos as estimativas clássicas e Bayesianas para este modelo.

Tabela 3.32 Estimativas para os parâmetros.

\begin{tabular}{c|c|c|c|c}
\hline Parâmetro & EMV & $\begin{array}{c}\text { Intervalo de } \\
\text { Confiança } \\
(95 \%)\end{array}$ & $\begin{array}{c}\text { Média a } \\
\text { posteriori }\end{array}$ & $\begin{array}{c}\text { Intervalo de } \\
\text { Credibilidade } \\
(95 \%)\end{array}$ \\
\hline \hline$\beta_{0}$ & 3,755 & $(1,815 ; 5,695)$ & 3,88 & $(1,95 ; 5,923)$ \\
\hline$\beta_{1}$ & $-0,145$ & $(-1,046 ; 0,756)$ & $-0,125$ & $(-1,014 ; 0,838)$ \\
\hline$\beta_{2}$ & $-0,648$ & $(-1,344 ; 0,047)$ & $-0,66$ & $(-1,379 ; 0,032)$ \\
\hline$\beta_{3}$ & $-1,635$ & $(-2,451 ;-0,855)$ & $-1,621$ & $(-2,4 ;-0,833)$ \\
\hline$\beta_{4}$ & $-0,019$ & $(-0,046 ; 0,008)$ & $-0,02$ & $(-0,050 ; 0,007)$ \\
\hline$\sigma$ & & & & \\
\hline
\end{tabular}

Tabela 3.33 Valores obtidos para $\sqrt{R}$ no critério de convergência de Gelman e Rubin para os parâmetros.

\begin{tabular}{c|c}
\hline Parâmetro & $\sqrt{R}$ \\
\hline$\beta_{0}$ & 1,01 \\
\hline$\beta_{1}$ & 1,00 \\
\hline$\beta_{2}$ & 1,00 \\
\hline$\beta_{3}$ & 1,00 \\
\hline$\beta_{4}$ & 1,01 \\
\hline$\sigma$ & 1,00 \\
\hline
\end{tabular}

Pela Tabela 3.33 observamos a convergência dos parâmetros usando o critério de Gelman e Rubin (1992).

Na Figura abaixo temos os gráficos a posteriori de cada parâmetro.
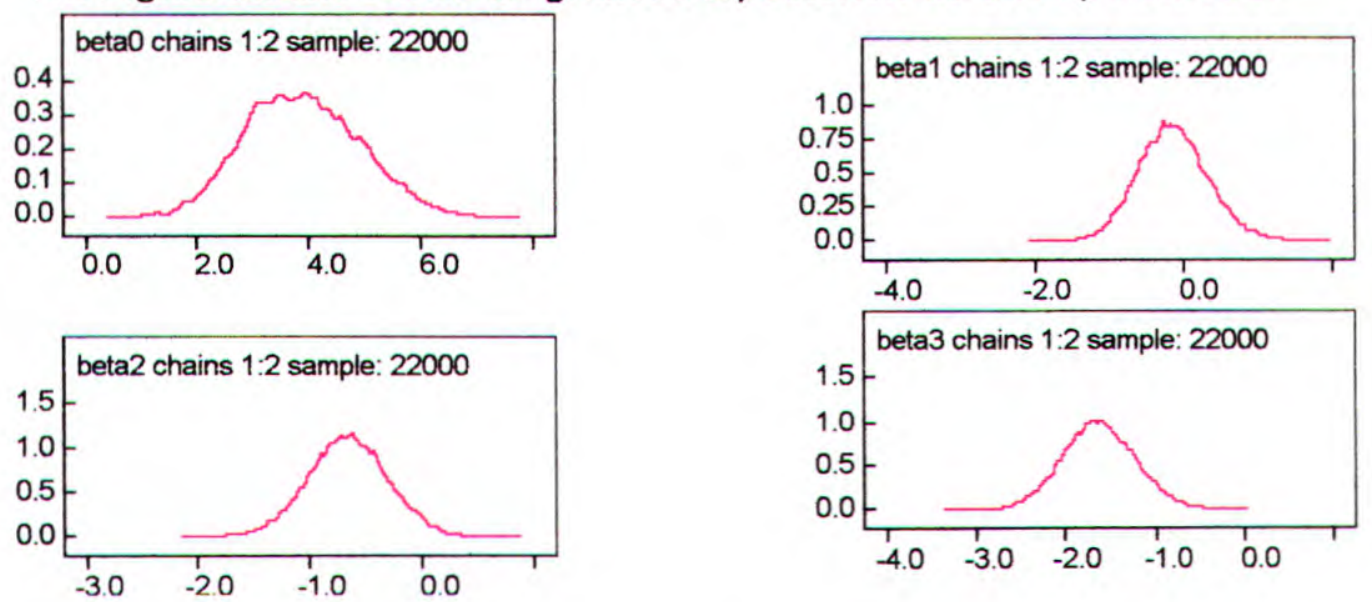

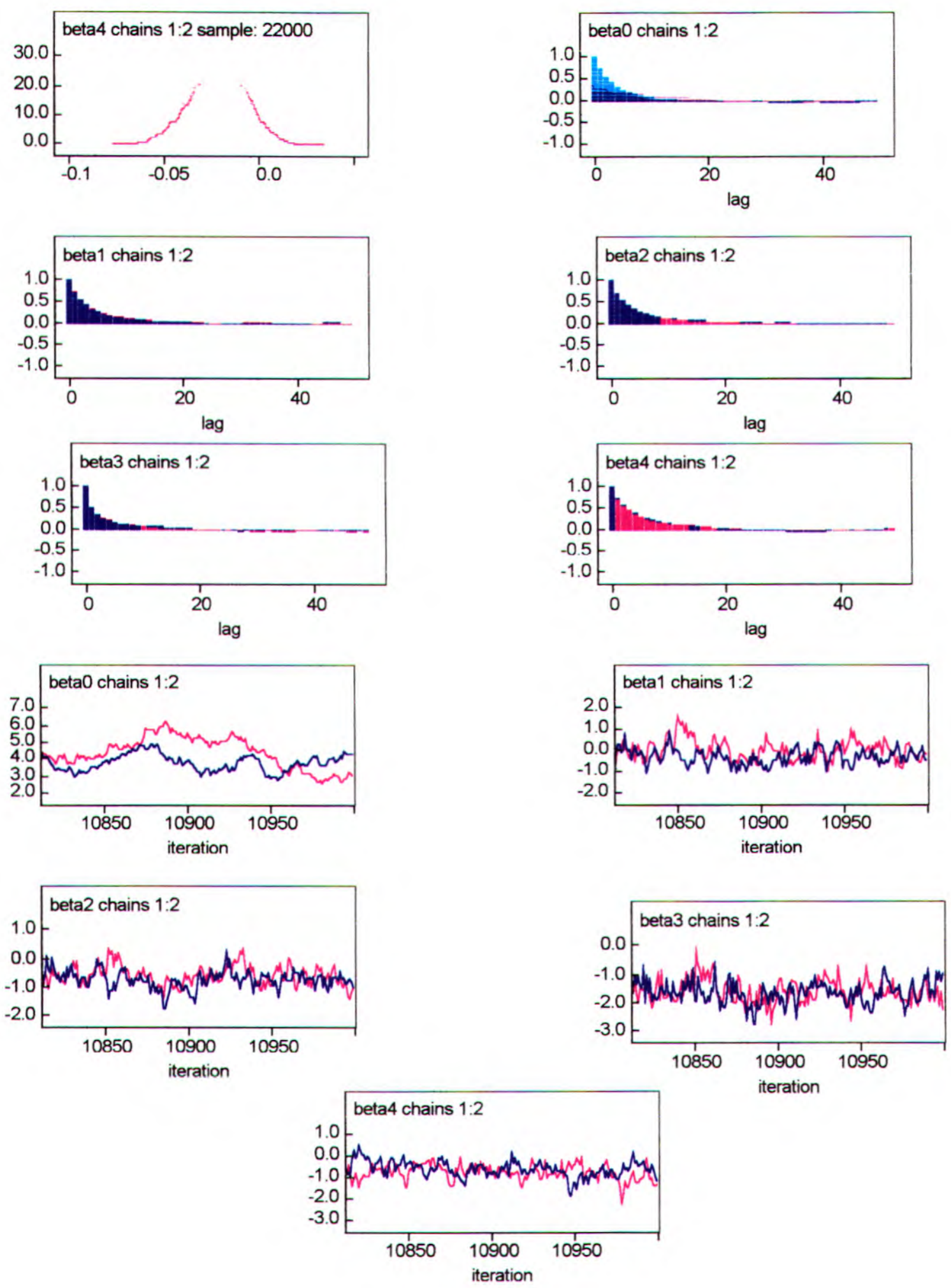

Figura 3.16: Gráficos a posteriori dos parâmetros para o modelo exponencial com os dados4.

Observando os gráficos de auto-correlação dos parâmetros para este modelo, temos que diferentemente dos anteriores, temos correlações até entre 20 números seguidos. Então, descartamos os primeiros 1000 valores da amostra de 11000 para cadeia, e depois selecionamos de 20 em 20 totalizando um final de 500 amostras para cada parâmetro. 


\subsubsection{Modelo de Regressão Weibull}

Para este conjunto de dados com mais de uma covariável também ajustamos um modelo de regressão de Weibull. As estimativas de máxima verossimilhança foram feitas de acordo com a seção 2.2.1 para dados censurados. As distribuiçōes a priori utilizadas para este modelo são dadas na seção 2.2.2.2 com os seguintes valores para os hiperparâmetros: $a=10, b=10, c_{j}=0, d_{j}=1000$, onde $\circ \mathrm{j}$ indexa $\circ$ valor dos coeficientes de regressão.

Os resultados obtidos para as estimativas clássicas e Bayesianas dos seis paràmetros de interesse são dados abaixo.

Tabela 3.34 Estimativas para os parâmetros.

\begin{tabular}{c|c|c|c|c}
\hline Paràmetro & EMV & $\begin{array}{c}\text { Intervalo de } \\
\text { Confiança } \\
(95 \%)\end{array}$ & $\begin{array}{c}\text { Média a } \\
\text { posteriori }\end{array}$ & $\begin{array}{c}\text { Intervalo de } \\
\text { Credibilidade } \\
(95 \%)\end{array}$ \\
\hline$\beta_{0}$ & 3,528 & $(1,756 ; 5,299)$ & 3,878 & $(1,727 ; 6,028)$ \\
\hline$\beta_{1}$ & $-0,148$ & $(-0,945 ; 0,649)$ & $-0,115$ & $(-1,042 ; 0,812)$ \\
\hline$\beta_{2}$ & $-0,586$ & $(-1,211 ; 0,392)$ & $-0,654$ & $(-1,373 ; 0,065)$ \\
\hline$\beta_{3}$ & $-1,544$ & $(-2,255 ;-0,832)$ & $-1,609$ & $(-2,420 ;-0,797)$ \\
\hline$\beta_{4}$ & $-0,017$ & $(-0,040 ; 0,006)$ & $-0,021$ & $(-0,048 ; 0,006)$ \\
\hline$\sigma$ & 0,885 & $(0,673 ; 1,096)$ & 0,980 & $(0,721 ; 1,238)$ \\
\hline
\end{tabular}

Na Tabela 3.34 notamos que os desvios das estimativas Bayesianas estão maiores que os da EMV, o que difere dos modelos ajustados no caso de uma única covariável. É importante salientar que os intervalos de confiança baseados na aproximação normal assintótica dos estimadores de máxima verossimilhança podem năo ser muito precisos, especialmente com dados censurados.

É interessante observar novamente, que os intervalos clássico e Bayesiano 'cobrem' o valor 1 para o caso de $\sigma$. Isto significa que o modelo Weibull se comporta de forma análoga ao exponencial. 
Tabela 3.35 Valores obtidos para $\sqrt{ } R$ no critério de convergência de Gelman e Rubin para os parâmetros.

\begin{tabular}{c|c}
\hline Parâmetro & $\sqrt{R}$ \\
\hline$\beta_{0}$ & 1,01 \\
\hline$\beta_{1}$ & 1,00 \\
\hline$\beta_{2}$ & 1,00 \\
\hline$\beta_{3}$ & 1,00 \\
\hline$\beta_{4}$ & 1,01 \\
\hline$\sigma$ & 1,00 \\
\hline
\end{tabular}

Novamente observamos a convergência de todos os parâmetros.

Os gráficos das distribuições a posteriori marginais são mostrados abaixo pela Figura 3.17.
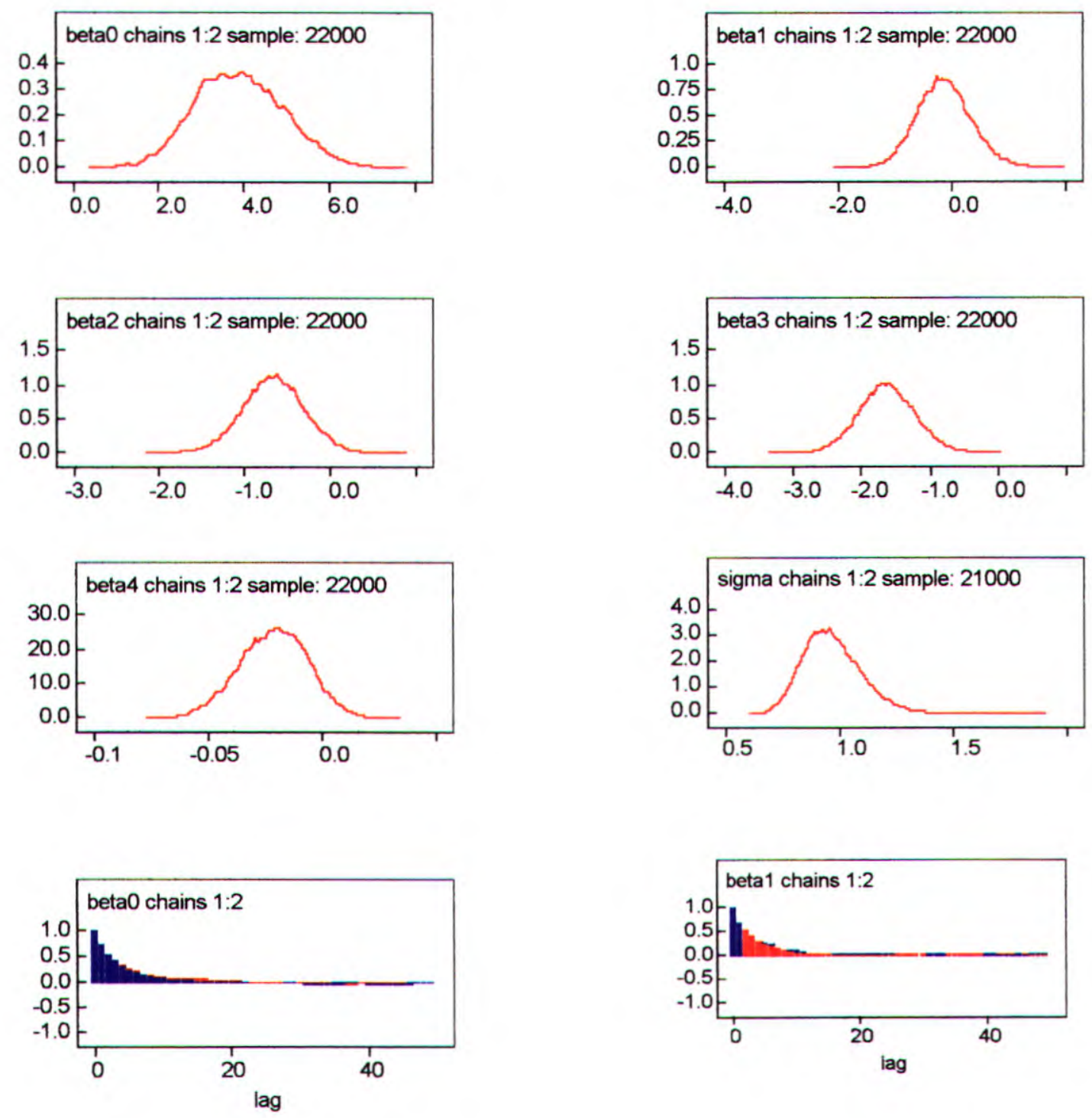

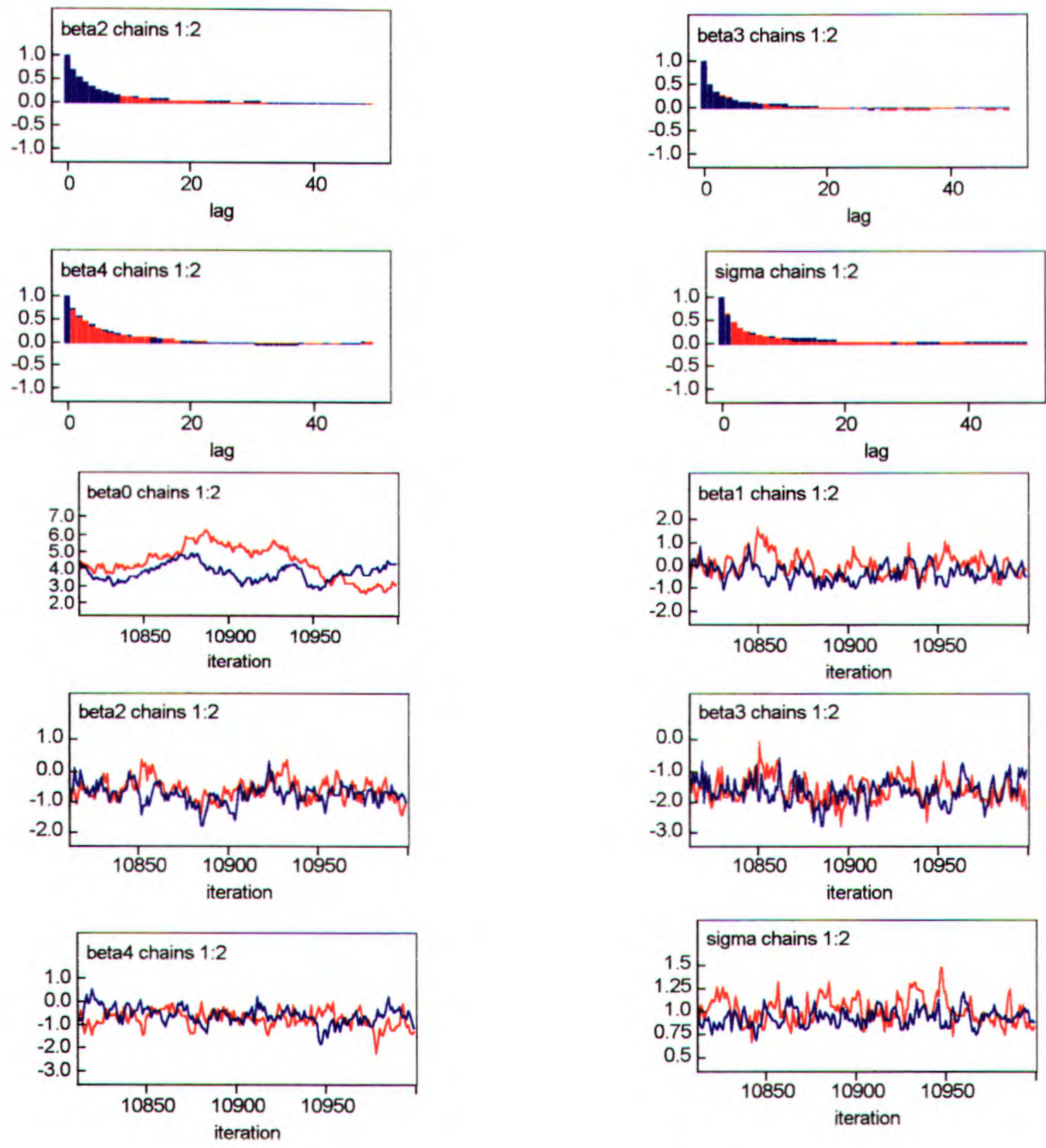

Figura 3.17: Gráficos a posteriori dos parâmetros para o modelo Weibull dos dados 4.

Como dito anteriormente, o modelo Weibull se comporta como o Exponencial e assim, os gráficos também. Em vista disto, as amostra foram obtidas da mesma maneira para os dois modelos.

\subsubsection{Modelo de Regressão Logística}

Agora consideramos o modelo de regressão logística dado na seção 2.31 e assumimos as mesmas distribuições a priori consideradas para o modelo de regressão Weibull e Exponencial. 
As estimativas clássicas e Bayesianas são dadas na Tabela 3.36.

Tabela 3.36 Estimativas para os parâmetros.

\begin{tabular}{c|c|c|c|c}
\hline Parâmetro & EMV & $\begin{array}{c}\text { Intervalo de } \\
\text { Confiança } \\
(95 \%)\end{array}$ & $\begin{array}{c}\text { Média a } \\
\text { posteriori }\end{array}$ & $\begin{array}{c}\text { Intervalo de } \\
\text { Credibilidade } \\
(95 \%)\end{array}$ \\
\hline$\beta_{0}$ & 3,102 & $(1,236,4,968)$ & 2,893 & $(1,518,4,272)$ \\
\hline$\beta_{1}$ & $-0,125$ & $(-0,938,0,688)$ & $-0,067$ & $(-0,825,0,682)$ \\
\hline$\beta_{2}$ & $-0,805$ & $(-1,496,-0,113)$ & $-0,647$ & $(-1,359,0,055)$ \\
\hline$\beta_{3}$ & $-1,766$ & $(-2,599,-0,933)$ & $-1,646$ & $(-2,768,-0,649)$ \\
\hline$\beta_{4}$ & $-0,015$ & $(-0,042,0,012)$ & $-0,034$ & $(-0,056,-0,012)$ \\
\hline$\sigma$ & 0,715 & $(0,547,0,883)$ & 0,898 & $(0,805,1,007)$ \\
\hline
\end{tabular}

Ao contrário do modelo Weibull, observamos que as estimativas Bayesianas foram mais precisas do que as clássicas. Novamente é importante salientar que as estimativas por intervalo clássicas foram obtidas a partir de resultados assintóticos.

Neste modelo, notamos também que as estimativas não estão tão próximas como ocorria anteriormente e isto pode estar acontecendo pois temos dados censurados e a aproximação assintótica utilizada pelo procedimento nlp pode não ser muito boa.

Tabela 3.37 . Valores para $\sqrt{ } R$ no critério de convergência de Gelman e Rubin para os parâmetros.

\begin{tabular}{c|c}
\hline Parâmetro & $\sqrt{R}$ \\
\hline$\beta_{0}$ & 1,01 \\
\hline$\beta_{1}$ & 1,00 \\
\hline$\beta_{2}$ & 1,00 \\
\hline$\beta_{3}$ & 1,00 \\
\hline$\beta_{4}$ & 1,01 \\
\hline$\sigma$ & 1,00 \\
\hline
\end{tabular}

Todos os parâmetros convergiram sem problemas (ver Tabela 3.37).

Os gráficos das distribuições a posteriori marginais são mostrados na Figura 3.18, 

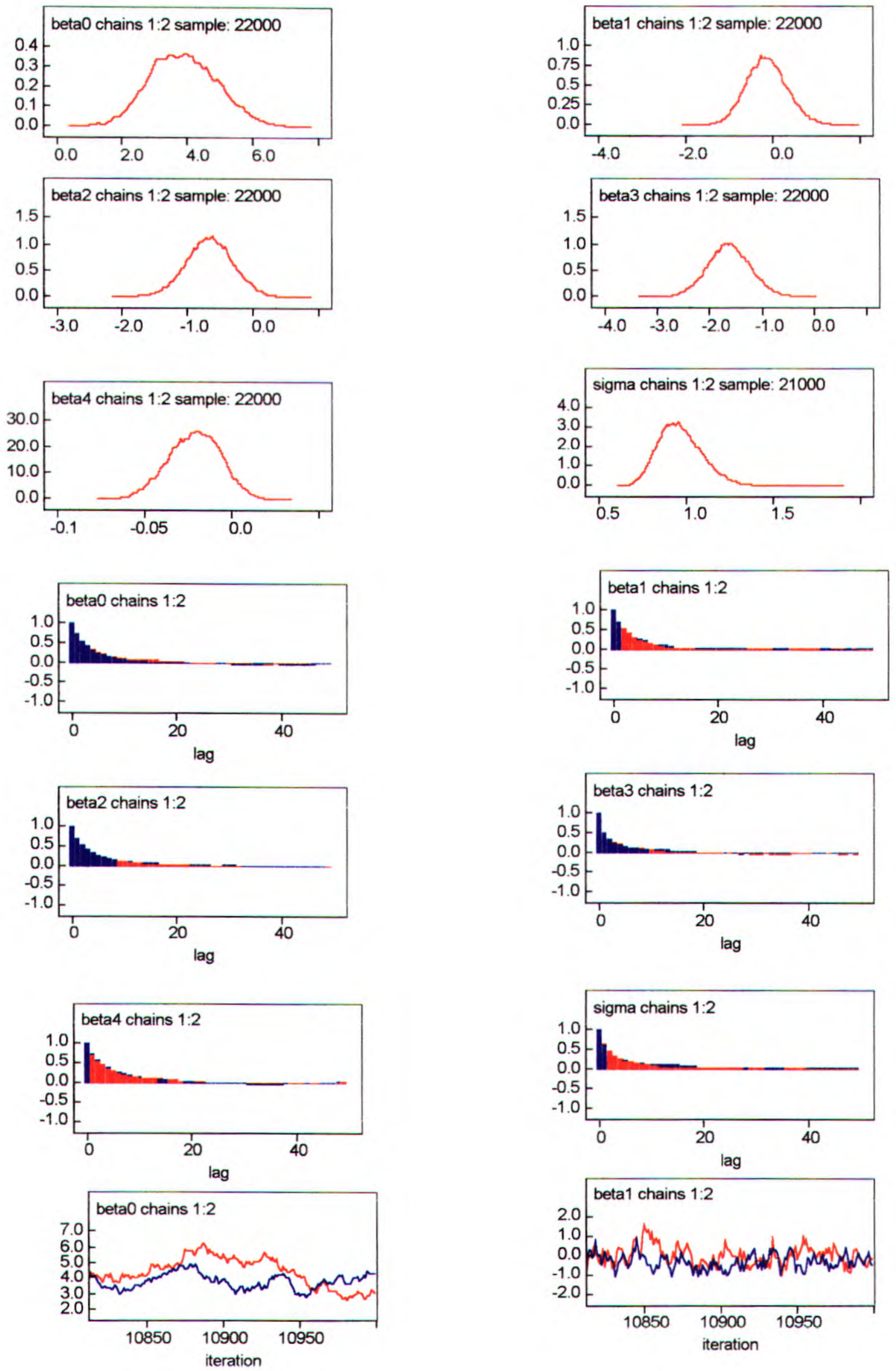

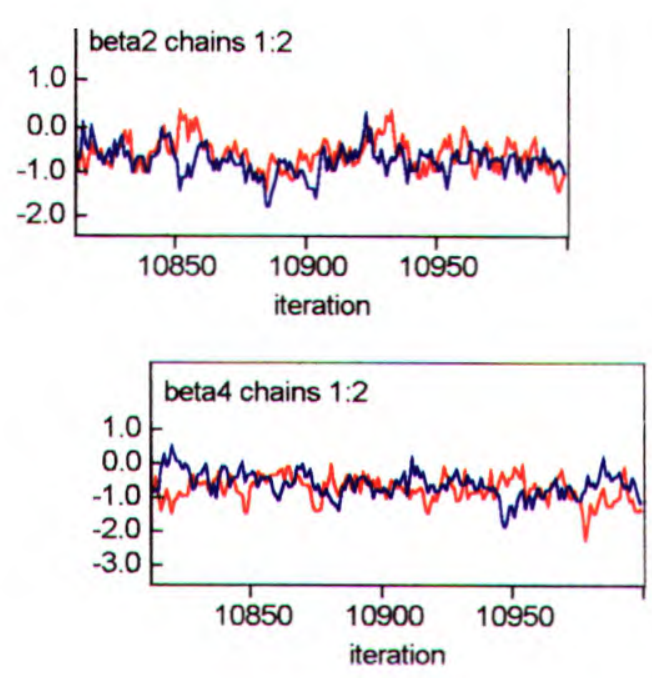
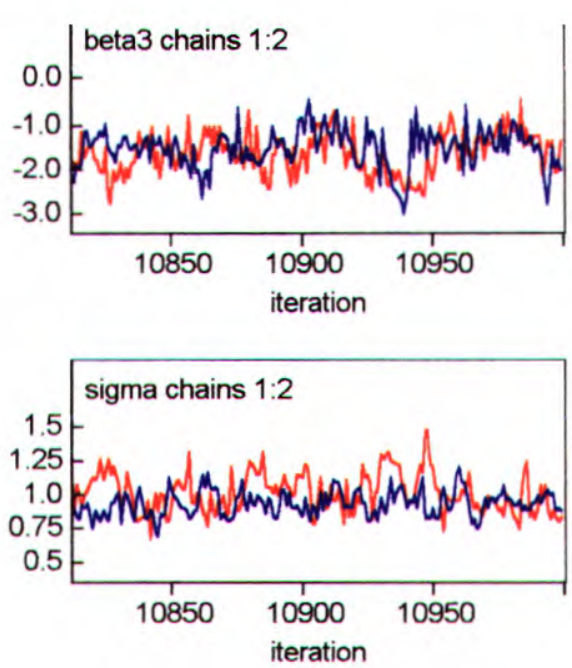

Figura 3.18: Gráficos a posteriori dos parâmetros para o modelo logístico para os dados4.

Novamente as cadeias tiveram um comportamento como o do modelo exponencial. As auto-correlações foram até 20 valores consecutivos.

\subsubsection{Modelo de Regressão Log- Normal}

Para finalizar esses exemplos de regressão log-lineares vamos ajustar um modelo log-normal para os dados 4. Novamente utilizamos as mesmas distribuições a priori dadas nos modelos Weibull.

As estimativas clássicas e Bayesianas são dadas naTabela 3.38.

Tabela 3.38 Estimativas para os parâmetros.

\begin{tabular}{c|c|c|c|c}
\hline Parâmetro & EMV & $\begin{array}{c}\text { Intervalo de } \\
\text { Confiança } \\
(95 \%)\end{array}$ & $\begin{array}{c}\text { Média a } \\
\text { Posteriori }\end{array}$ & $\begin{array}{c}\text { Intervalo de } \\
\text { Credibilidade } \\
(95 \%)\end{array}$ \\
\hline \hline$\beta_{0}$ & 3,383 & $(1,550 ; 5,215)$ & 3,234 & $(1,906 ; 4,563)$ \\
\hline$\beta_{1}$ & $-0,198$ & $(-1,064 ; 0,668)$ & $-0,154$ & $(-0,989 ; 0,679)$ \\
\hline$\beta_{2}$ & $-0,899$ & $(-1,611 ;-0,186)$ & $-0,874$ & $(-1,577 ;-0,185)$ \\
\hline$\beta_{3}$ & $-1,857$ & $(-2,723 ;-0,990)$ & $-1,84$ & $(-2,661 ;-1,025)$ \\
\hline$\beta_{4}$ & $-0,018$ & $(-0,043 ; 0,007)$ & $-0,016$ & $(0,036 ; 0,004)$ \\
\hline$\sigma$ & 1,263 & $(1,000 ; 1,525)$ & 1,343 & $(1,087 ; 1,685)$ \\
\hline \hline
\end{tabular}

As estimativas log-normais foram mais próximas que as log-logísticas. Não observamos na Tabela 3.38 nenhum parâmetro com estimativas muito diferentes 
entre si.

Tabela 3.39 . Valores para $\sqrt{ } R$ no critério de convergência de Gelman e Rubin para os parâmetros.

\begin{tabular}{c|c}
\hline Parâmetro & $\sqrt{R}$ \\
\hline \hline$\beta_{0}$ & 1,01 \\
\hline$\beta_{1}$ & 1,00 \\
\hline$\beta_{2}$ & 1,00 \\
\hline$\beta_{3}$ & 1,00 \\
\hline$\beta_{4}$ & 1,01 \\
\hline$\sigma$ & 1,00 \\
\hline
\end{tabular}

Observar que houve convergência para todos os parâmetros como podemos observar pela Tabela 3.39.

Os gráficos abaixo mostram as distribuições a posteriori de cada parâmetro.
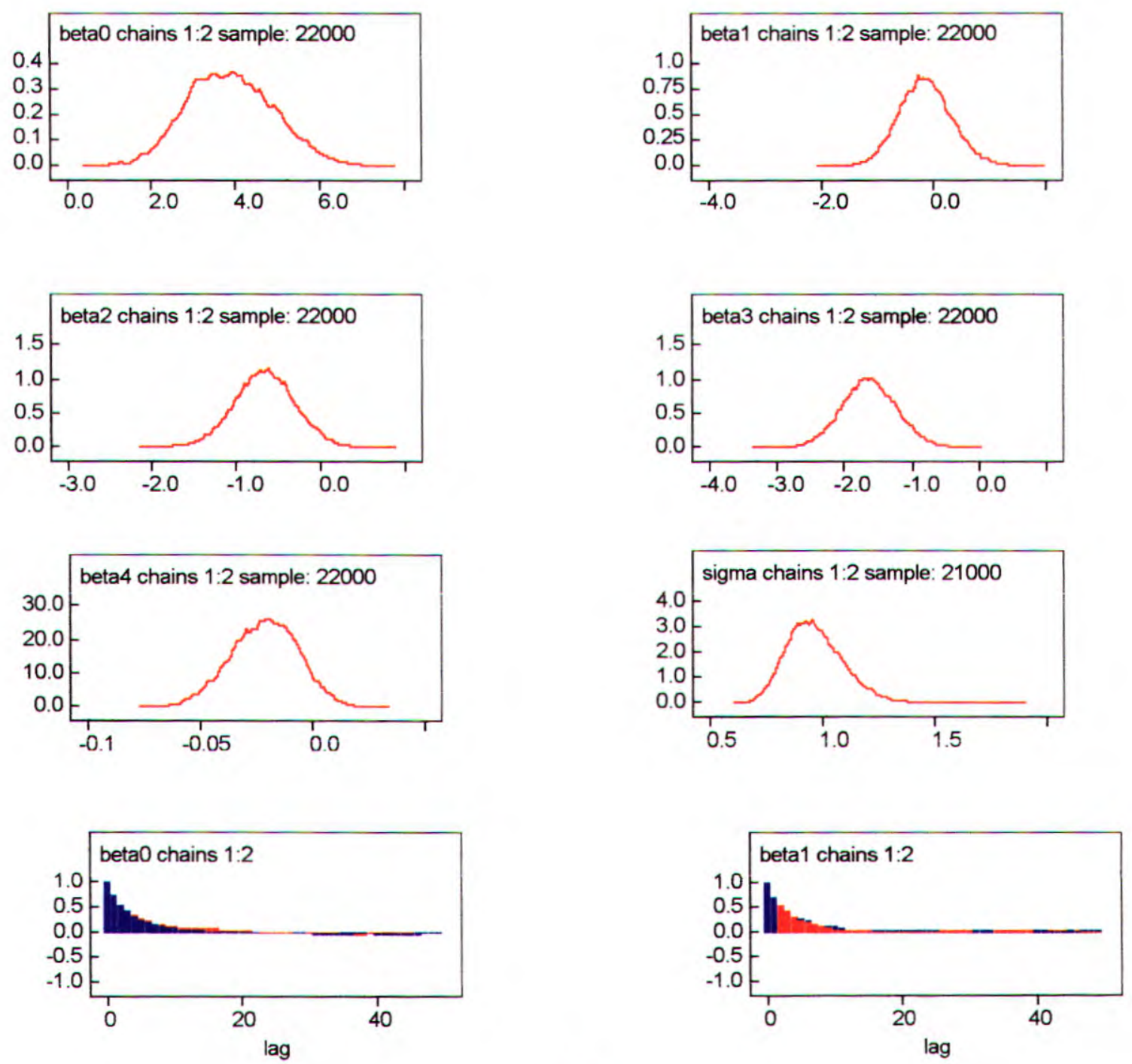

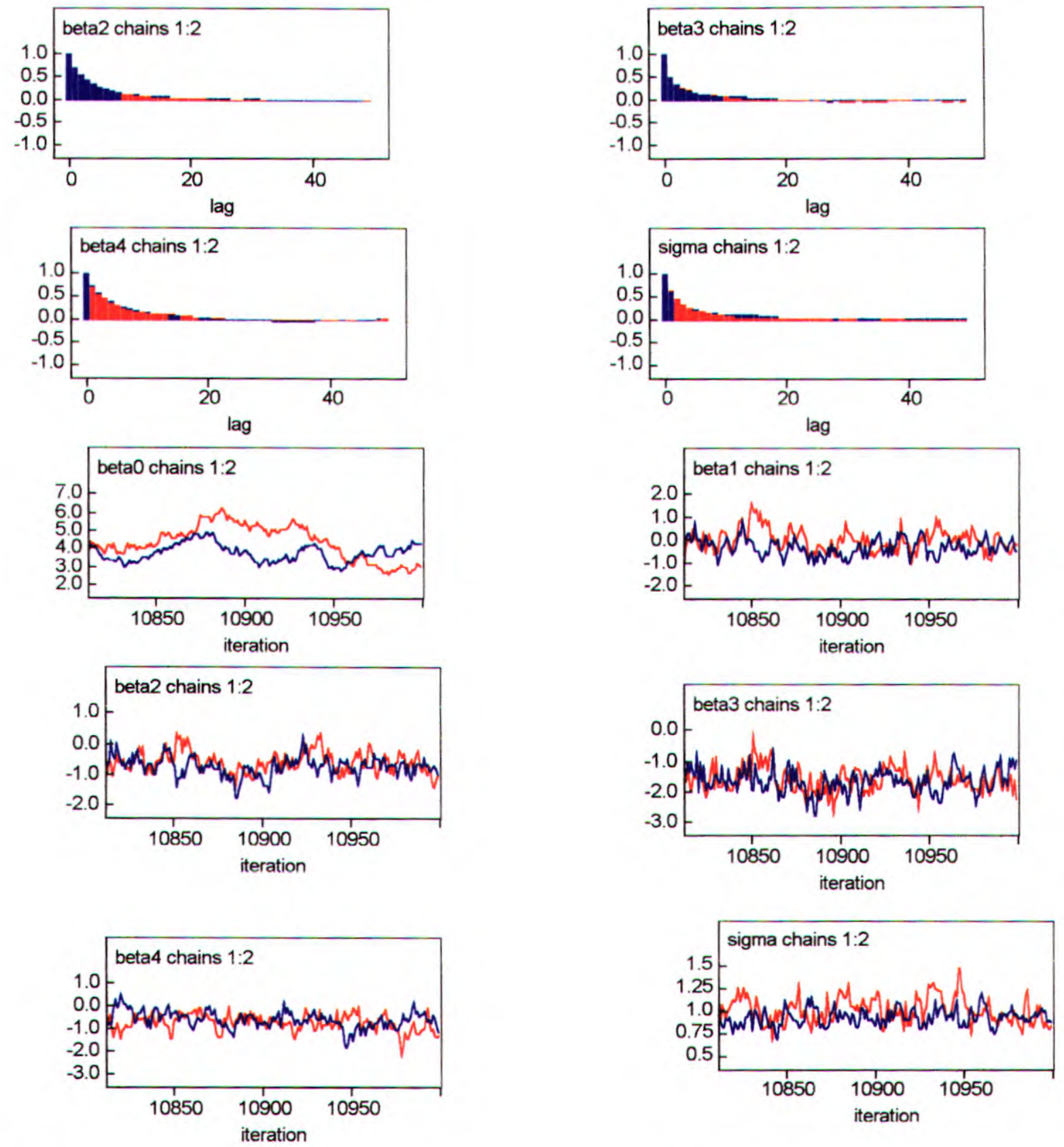

Figura 3.19: Gráficos a posteriori dos parâmetros para o modelo Log- normal dos dados 4 .

O conjunto de dados número quatro teve um comportamento de cadeias praticamente igual para todos os modelos ajustados. 


\section{CAPÍTULO 4: Mistura de Distribuições}

Agora vamos introduzir o problema de mistura finita de distribuições paramétricas. (ver por exemplo, Titterington et al. 1985).

O uso de distribuições com misturas de distribuições pode ser necessário quando a distribuição de sobrevivência é multimodal, por exemplo.

Exemplos são introduzidos neste capítulo para ilustrar a idéia de mistura de distribuições.

\subsection{Introdução aos Modelos de Mistura de Distribuições}

Suponhamos que uma amostra de tamanho n de uma variável aleatória $\mathrm{X}, \mathrm{X}_{1}$, $X_{2}, \ldots, X_{n}$, é obtida de uma população formada por $J$ sub-populações com densidades: $f\left(x / \theta_{j}\right) \mathrm{j}=1,2, \ldots, \mathrm{J}$. Suponhamos ainda que não temos conhecimento de qual sub-população a variável $X_{i}$ é proveniente. Nesta situação, é natural supor que cada variável $X_{i}$ é pertencente a uma sub-população com densidade $f\left(x_{i} / \theta_{j}\right)$ com probabilidade $\mathrm{p}_{\mathrm{j}}$ tal que $\sum_{j=1}^{J} p_{i j}=1$. Neste caso, o modelo misto é definido como:

$$
f(x / \theta)=\sum_{i=1}^{J} p_{j} f\left(x / \theta_{j}\right)
$$

onde $\theta=\left(\theta_{1}, \ldots, \theta_{J}\right)$ e $p=\left(p_{1}, \ldots, p_{J}\right)$ são vetores desconhecidos.

Nosso objetivo é obter informação sobre $\theta$ baseado na amostra $X_{1}, \ldots, X_{n}$.

A função de verossimilhança no caso de mistura é dada por:

$$
L(\theta, p)=\prod_{i=1}^{n} \sum_{j=1}^{J} p_{j} f\left(x_{i} \mid \theta_{j}\right)
$$


A distribuição a posteriori é dada por,

$$
\pi(\theta, p \mid X) \propto \pi(\theta, p) L(\theta, p)
$$

Geralmente, independente da distribuição a priori que será utilizada, existe uma grande dificuldade computacional na obtenção da distribuição a posteriori devido à complexidade da função de verossimilhança.

\subsubsection{Princípio Básico baseado em amostra ampliada}

Usamos esse princípio para ampliar os dados com dados latentes $Z$ para simplificar a forma da distribuição a posteriori.

Definir uma variável não observável(ou latente) da forma:

$Z_{i j}=\left\{\begin{array}{l}1, \mathrm{j} \text { - ésima observação foi gerada da população } \mathrm{j} \\ 0, \text { caso contrário }\end{array}\right.$

onde $\sum_{j=1}^{J} Z_{i j}=1$, para $i=1, \ldots, \mathrm{ne}$

$$
q_{i j}=P\left(Z_{i j}=1 \mid \theta, p, Z\right)=\frac{p_{j} f\left(x_{i} \mid \theta_{j}\right)}{\sum_{j=1}^{J} p_{j} f\left(x_{i} \mid \theta_{j}\right)}
$$

isto $\dot{e}, Z_{1} \mid X, p, \theta \sim \operatorname{Mult}\left(1 ; q_{i 1}, \ldots, q_{i}\right)$, onde $Z_{i}=\left(Z_{i 1}, \ldots, Z_{15}\right)$.

A função de verosssimilhança baseada em dados ampliados é dada por:

$$
L_{A}(\theta, p)=\prod_{i=1}^{n} \prod_{j=1}^{J}\left(p_{j} f\left(x_{i} \mid \theta_{j}\right)\right)^{Z_{l}}
$$

Assim, conseguimos eliminar o somatório da verossimilhança que dificultava a obtenção das inferências de interesse.

A partir dessa simplificaçăo temos o seguinte algoritmo com dados ampliados: 
Dado um valor inicial $\left(p^{0}, \theta^{0}\right)$, o algoritmo na etapa $m$ é dado por, Primeiro Passo: Gerar $Z_{i}^{(m)} \sim$ Mult $\left[1 ; q_{i 1}{ }^{(m)}, \ldots, q_{i j}{ }^{(m)}\right]$, dado $\theta^{(m)}, p{ }^{(m)}$ e X, para $i=1, \ldots, n$.

Segundo Passo: Gerar:

$$
\begin{aligned}
& p^{(m+1)} \sim \pi\left(p \mid X, Z^{(m)}\right) \\
& \theta^{(m+1)} \sim \pi\left(\theta \mid X, Z^{(m)}\right)
\end{aligned}
$$

Terceiro Passo: Repetir os outros passos até iteração desejada.

Após (M) etapas ("burn in"), os vetores $\left(\theta^{(M+k)}, \ldots, \theta^{(M+k N)}\right)$ e $\left(p^{(M+k)}, \ldots, p^{(M+k N)}\right)$ podem ser considerados aproximadamente como uma amostra de tamanho $\mathbf{N}$ da posteriori marginal $\pi(\theta \mid X)$ e $\pi(p \mid X)$, respectivamente.

As distribuições marginais de $\theta$ e $p$ dado $X$ podem ser estimadas por,

$$
\begin{aligned}
& \hat{\pi}(\theta \mid X)=\frac{\sum_{i=1}^{N} \pi\left(\theta \mid X, Z^{(M+k l)}\right)}{N} \\
& \hat{\pi}(p \mid X)=\frac{\sum_{i=1}^{N} \pi\left(p \mid X, Z^{(M+k l)}\right)}{N}
\end{aligned}
$$

As justificativas destes resultados podem ser encontradas em Tanner \& Wong (1987) ou Diebolt \& Roberts (1994).

Neste capítulo, ao contrário do capítulo 2, não faremos as Análises Clássicas e Bayesianas separadamente. A Análise Clássica é feita a partir da função de verossimilhança que será mostrada em todos os modelos. Em geral, maximizamos o logaritmo da verossimilhança no SAS para obter os resultados clássicos. Com exceção do modelo de mistura de duas normais que conseguimos fazer no WinBugs, todos os outros modelos foram feitos no $\mathrm{Ox}$. 


\subsection{Mistura de 2 Distribuições Weibull para $T$}

Em alguns casos observamos que a variável aleatória $\mathrm{T}$ tem uma distribuição dada por uma mistura de densidades de Weibull na forma (4.1), onde

$$
f_{j}\left(t \mid \alpha_{j}, \beta_{j}\right)=\left(\frac{\beta_{j}}{\alpha_{j}}\right)\left(\frac{t}{\alpha_{j}}\right)^{\beta_{j}-1} \exp \left\{-\left(\frac{t}{\alpha_{j}}\right)^{\beta_{j}}\right\}
$$

Utilizando o princípio de amostras ampliadas, a função de verossimilhança é dada pela equação (4.6).

Neste caso, temos,

$$
\begin{aligned}
& q_{i 1}=\frac{p_{1} f_{1}\left(t \mid \alpha_{1}, \beta_{1}\right)}{\sum_{j=1}^{2} p_{j} f_{j}\left(t \mid \alpha_{j}, \beta_{j}\right)}, \mathrm{i}=1, \ldots, \mathrm{n} \text { e } \mathrm{j}=1,2 . \\
& q_{i 2}=1-q_{i 1}
\end{aligned}
$$

Considerar as seguintes distribuições a priori:

$$
\begin{array}{lll}
\text { (i) } \beta_{j} \sim N\left(c_{j}, d^{2}\right) & , \mathrm{c}_{\mathrm{j}}, \mathrm{d}_{\mathrm{j}} \text { conhecidos } & \mathrm{j}=1,2 \\
(\text { ii }) \alpha_{j} \sim N\left(e_{j}, f_{j}^{2}\right) & , \mathrm{e}_{\mathrm{j}}, \mathrm{f}_{\mathrm{j}} \text { conhecidos } & \mathrm{j}=1,2 \\
\text { (iii) } p \sim B(a, b) & \text {, a b conhecidos } &
\end{array}
$$

Quando não temos covariáveis nem censuras, o logaritmo da verossimilhança é dado por,

$$
\begin{aligned}
l= & \sum_{i=1}^{n} q_{i 1} \log p_{1}+\sum_{i=1}^{n} q_{i 1} \log \beta_{1}-\beta_{1} \sum_{i=1}^{n} q_{i 1} \log \alpha_{1}+\left(\beta_{1}-1\right) \sum_{i=1}^{n} q_{i 1} \log t_{i}-\sum_{i=1}^{n} q_{i 1}\left(\frac{t_{i}}{\alpha_{1}}\right)^{\beta_{1}}+ \\
& \sum_{i=1}^{n} q_{i 2} \log p_{2}+\sum_{i=1}^{n} q_{i 2} \log \beta_{2}-\beta_{2} \sum_{i=1}^{n} q_{i 2} \log \alpha_{2}+\left(\beta_{2}-1\right) \sum_{i=1}^{n} q_{i 2} \log t_{i}-\sum_{i=1}^{n} q_{i 2}\left(\frac{t_{i}}{\alpha_{2}}\right)^{\beta_{2}}
\end{aligned}
$$


Assim, os logaritmos das distribuiçōes condicionais para o algoritmo ARMS são dadas por,

$$
\begin{aligned}
& l_{\beta_{1}}=\sum_{i=1}^{n} q_{i 1} \log \beta_{1}-\beta_{1} \sum_{i=1}^{n} q_{i 1} \log \alpha_{1}+\left(\beta_{1}-1\right) \sum_{i=1}^{n} q_{i 1} \log t_{i}-\sum_{i=1}^{n} q_{i 1}\left(\frac{t_{i}}{\alpha_{1}}\right)^{\beta_{1}}+\log \pi\left(\beta_{1}\right) \\
& l_{\alpha_{1}}=\beta_{1} \sum_{i=1}^{n} q_{i 1} \log \alpha_{1}-\sum_{i=1}^{n} q_{i n}\left(\frac{t_{i}}{\alpha_{1}}\right)^{\beta_{1}}+\log \pi\left(\alpha_{1}\right)
\end{aligned}
$$

As distribuiçōes condicionais para os outros parâmetros da mistura podem ser encontradas trocando os índices 1 por 2 . $O$ parâmetro $p$ pode ser gerado pelo algoritmo Gibbs diretamente.

$$
p_{1} \sim \operatorname{Beta}\left(1, \sum_{i=1}^{n} q_{i 1}\right)
$$

\subsection{Mistura de 3 Distribuições Weibull para $T$}

Considerar agora uma mistura de 3 distribuiçōes Weibull.

Neste caso, temos $j=1,2$ ou 3. Assim, temos,

$$
\begin{aligned}
& q_{i 1}=\frac{p_{1} f_{1}\left(t \mid \alpha_{1}, \beta_{1}\right)}{\sum_{j=1}^{3} p_{j} f_{j}\left(t \mid \alpha_{j}, \beta_{j}\right)} \\
& q_{i 2}=\frac{p_{2} f_{2}\left(t \mid \alpha_{2}, \beta_{2}\right)}{\sum_{j=1}^{3} p_{j} f_{j}\left(t \mid \alpha_{j}, \beta_{j}\right)} \\
& q_{13}=1-q_{i 1}-q_{12}
\end{aligned}
$$

Utilizamos as mesmas distribuiçōes a priori para $\alpha$ e $\beta$ dadas em (4.10) mas 
com j variando de 1 a 3 . Para o parâmetro $p$, consideramos uma distribuição a priori Dirichlet,

$p \sim \operatorname{Dirichlet}(a, b, c)$ com a, b e c conhecidos.

Novamente, o parâmetro $p$ pode ser gerado diretamente por Gibbs e os logaritmos das distribuições condicionais para os outros parâmetros são dadas pelas expressões abaixo,

$$
\begin{aligned}
& l_{\beta_{j}}=\sum_{i=1}^{n} q_{i j} \log \beta_{j}-\beta_{j} \sum_{i=1}^{n} q_{i j} \log \alpha_{j}+\left(\beta_{j}-1\right) \sum_{i=1}^{n} q_{i j} \log t_{i}-\sum_{i=1}^{n} q_{i j}\left(\frac{t_{i}}{\alpha_{j}}\right)^{\beta_{j}}+\log \pi\left(\beta_{j}\right) \\
& l_{\alpha_{j}}=-\beta_{j} \sum_{i=1}^{n} q_{i j} \log \alpha_{j}-\sum_{i=1}^{n} q_{i j}\left(\frac{t_{i}}{\alpha_{j}}\right)^{\beta_{j}}+\log \pi\left(\alpha_{j}\right)
\end{aligned}
$$

\subsection{Mistura de 2 Distribuições Gama Generalizadas}

Assumimos agora um modelo de misturas com distribuiçōes Gamas Generalizadas. Como dito anteriormente, este é um supermodelo e uma mistura de distribuições Gamas Generalizadas poderia englobar vários tipos de mistura já que dependendo de um parâmetro $\mathrm{k}$ poderiamos ter diferentes distribuições usadas para analisar dados de sobrevivência.

Novamente consideramos a verossimilhança do tipo (4.6), com dados ampliados. A função de verossimilhança é dada pela expressão abaixo:

$$
L_{A}=\prod_{i=1}^{n}\left[p_{1}^{z_{11}} \frac{\beta^{z_{n_{1}}}}{\Gamma\left(k_{1}\right)^{z_{11}}} \lambda_{1}^{z_{14} k_{1} \beta_{1}}\left(t_{i}\right)^{\left(k_{1} \beta_{1}-1\right) z_{t 1}} \exp \left[-z_{i 1}\left(\lambda_{1} t_{i}\right)^{\beta_{1}}\right] p_{2}^{z_{i 2}} \frac{\beta_{2}^{z_{i 2}}}{\Gamma\left(k_{2}\right)^{z i 2}} \lambda_{2}^{k_{2} \beta_{2} z_{i 2}}\left(t_{i}\right)^{\left(k_{2} \beta_{2}-1\right) z_{12}} \exp \left[-z_{i 2}\left(\lambda_{2} t_{2}\right)^{\beta_{2}}\right]\right]
$$

Isto é, 


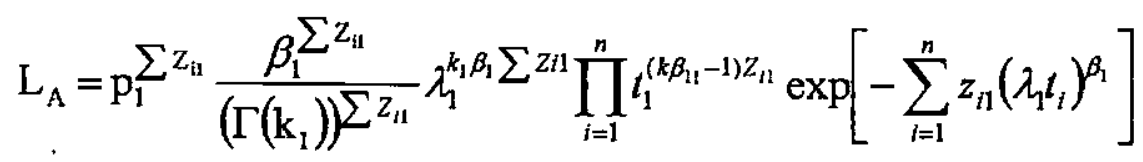

$$
\begin{aligned}
& \left(1-p_{1}\right)^{\sum z_{12}} \frac{\beta_{2}^{\sum z_{12}}}{\left(\Gamma\left(k_{2}\right)\right)^{\sum z_{i 2}}} \lambda_{2}^{k_{2} \beta_{2} \sum z_{12}} \prod_{i=1}^{n} t_{1}^{\left(k_{2} \beta_{2}-1\right) z_{12}} \exp \left[-\sum_{i=1}^{n} z_{i 2}\left(\lambda_{21} t_{i}\right)^{\beta_{2}}\right]
\end{aligned}
$$

Aplicando a função logaritmica na verossimilhança, obtemos os logaritmos das distribuições condicionais para o algoritmo Gibbs sampling:

$$
\begin{aligned}
& (i) l_{\beta_{j}}=\sum_{i=1}^{n} z_{i j} \log \beta_{j}+k_{j} \beta_{j} \sum_{i=1}^{n} z_{i j} \log \lambda_{j}+\left(k_{j} \beta_{j}-1\right) \sum_{i=1}^{n} z_{i j} \log \left(t_{i}\right)-\sum_{i=1}^{n} z_{i j}\left(\lambda_{j} t_{i}\right)^{\beta_{j}}+\log \pi\left(\beta_{j}\right) \\
& \text { (ii) } l_{\lambda_{j}}=k_{j} \beta_{j} \sum_{i=1}^{n} z_{i j} \log \left(\lambda_{j}\right)-\sum_{i=1}^{n} z_{i j}\left(\lambda_{j} t_{i}\right) \beta_{j}+\log \pi\left(\lambda_{j}\right) \\
& \text { (iii) } l_{k_{j}}=-\log \Gamma\left(k_{j}\right) \sum_{i=1}^{n} z_{i j}+k_{j} \beta_{j} \sum_{i=1}^{n} z_{i j} \log \left(\lambda_{j}\right)+\left(k_{j} \beta_{j}-1\right) \sum_{i=1}^{n} z_{i j} \log \left(t_{i}\right)
\end{aligned}
$$

É importante notar que o índice j nos mostra de que distribuição os dados pertencem mas as condicionais são similares para a mistura, assim, nas distribuições condicionais apresentadas acima temos o indice j que pode assumir os valores 1 ou 2 .

Assim, variando o indice $\mathrm{j}$ temos as condicionais para todos os parâmetros da mistura. Como nos modelos anteriores, o parâmetro $p$ é gerado diretamente pelo algoritmo Gibbs sampling.

\subsection{Mistura de 2 Distribuições Normais para $\log (T)$}

Em alguns casos, trabalhamos diretamente com o tempo de sobrevivência e em outros, com o logaritmo desse tempo.

Neste exemplo de mistura de Normais, temos que a variável latente é introduzida da mesma forma que nos modelos anteriores.

Assim, temos $Y=\log (T)$ e

$$
f(y)=\frac{1}{\sqrt{2 \pi} \sigma} \exp \left\{-\frac{1}{2 \sigma^{2}}(y-\mu)^{2}\right\}
$$


No modelo de mistura temos,

$$
f(y \mid \theta)=\sum_{i=1}^{2} p_{i} f(y \mid \theta)
$$

Considerar as distribuições a priori,

$$
\begin{array}{ll}
\text { (i) } \mu_{j} \sim N\left(c_{j}, d^{2}\right) & , \mathrm{c}_{\mathrm{j}}, \mathrm{d}_{\mathrm{j}} \text { conhecidos } \\
(\text { ii } \sigma \sim I G(a, b) & , \mathrm{a}, \mathrm{b} \text { conhecidos } \\
\text { (iii) } p \sim B(e, f) & , \mathrm{e}, \mathrm{f} \text { conhecidos }
\end{array}
$$

Utilizamos mistura de normais com variâncias iguais.

Com isso, a verossimilhança deste modelo é dada por:

$$
\begin{aligned}
L_{A} & =\prod_{i=1}^{n} \prod_{j=1}^{2}\left(p_{j} f\left(y \mid \theta_{j}\right)\right)^{z_{y}} \\
& \propto \mathrm{p}_{1} \sum^{\sum z_{1}}(\sigma)^{\sum z_{11}} \exp \left\{-\sum_{i=1}^{n} \frac{z_{i 1}}{2 \sigma^{2}}\left(y-\mu_{1}\right)^{2}\right\}\left(1-p_{1}\right)^{\sum z_{12}}\left(\sigma^{2}\right)^{\sum z_{b 2}} \exp \left\{-\sum_{i=1}^{n} \frac{z_{i 2}}{2 \sigma^{2}}\left(y-\mu_{2}\right)^{2}\right\}
\end{aligned}
$$

Aplicando o logaritmo nesta expressão podemos facilmente encontrar os logaritmos das distribuiçōes a posteriori condicionais para os parâmetros do modelo,

$$
\begin{aligned}
& (i) l_{\mu_{1}}=-\frac{1}{\sigma_{j}^{2}} \sum_{i=1}^{n} z_{i j}\left(y-\mu_{j}\right)^{2}+\ln \pi\left(\mu_{j}\right) \\
& (\text { ii }) l_{\sigma}=-\sum_{i=1}^{n} z_{i j} \ln (\sigma)-\frac{1}{\sigma^{2}} \sum_{i=1}^{n} z_{i j}\left(y-\mu_{j}\right)^{2}+\ln \pi(\sigma)
\end{aligned}
$$

Segundo Titterington et al. (1985), uma densidade é multimodal se ela possui mais de uma moda. No caso da densidade Normal, ele propões que esta será bimodal se e somente se $\left\lfloor\mu_{1}-\mu_{2}\right\rfloor \sigma>2$, onde $\left(\mu_{1}, \mu_{2}\right)$ são as médias e $\sigma^{2}$ é a variância comum. 


\subsection{Mistura de Distribuiçōes Normal e Exponencial}

Assumindo um modelo de mistura de Normal-Exponencial temos,

$f_{1}(y)=\beta_{0} \exp \left\{-\beta_{0} y\right\}$

$f_{2}(y)=\frac{1}{\sqrt{2 \pi} \sigma} \exp \left\{-\frac{1}{2 \sigma^{2}}(y-\mu)^{2}\right\}$

Neste caso, a função de verossimithança é dada por,

$$
\begin{aligned}
L_{A} & =\prod_{i=1}^{n} \prod_{j=1}^{2}\left(p_{j} f\left(y \mid \theta_{j}\right)\right)^{z_{u}} \\
& \propto \mathrm{p}_{1}^{\sum z_{i 1}} \beta_{o}^{\sum z_{11}} \exp \left\{-\sum_{i=1}^{n} z_{11} \beta_{0} y\right\}\left(1-p_{1}\right)^{\sum z_{i 2}}\left(\sigma_{2}^{2}\right)^{\sum z_{i b 2}} \exp \left\{-\sum_{i=1}^{n} \frac{z_{t 2}}{2 \sigma_{2}^{2}}\left(y-\mu_{2}\right)^{2}\right\}
\end{aligned}
$$

Os logaritmos das distribuições condicionais para o algoritmo ARMS sāo dadas por,

$$
\begin{aligned}
& \text { (i) } l_{\beta_{0}}=\sum_{i=1}^{n} z_{i 1} \ln \left(\beta_{0}\right)-\sum_{i=1}^{n} z_{i 1} \beta_{0} y_{i} \\
& \text { (ii) } l_{\mu}=-\sum_{i=1}^{n} \frac{z_{i 2}}{2 \sigma^{2}}\left(y_{i}-\mu\right)^{2}+\ln \pi(\mu) \\
& \text { (iii) } l_{\sigma}=-\sum_{i=1}^{n} z_{i 2} \ln (\sigma)-\sum_{i=1}^{n} \frac{z_{i 2}}{2 \sigma^{2}}\left(y_{i}-\mu\right)^{2}+\ln \pi(\sigma)
\end{aligned}
$$

O parâmetro $p$ pode ser gerado diretamente pelo algoritmo Gibbs, como foi observado previamente para outras misturas de distribuições.

\subsection{Exemplos de Mistura de Distribuições}

Agora vamos ilustrar como esses modelos de mistura podem ser utilizados para os dados 2 da Tabela 3.10 e para os dados 3 da Tabela 3.21. Para o primeiro e o último conjunto de dados citados no capitulo anterior não utilizamos esse tipo de modelagem. 


\subsubsection{Mistura nos dados 2}

Os dados 2 se referem ao tempo de falha de 60 aparelhos elétricos (dados na Tabela 3.10).

Histograma dos Tempos de Falha (Materiais Elétricos)

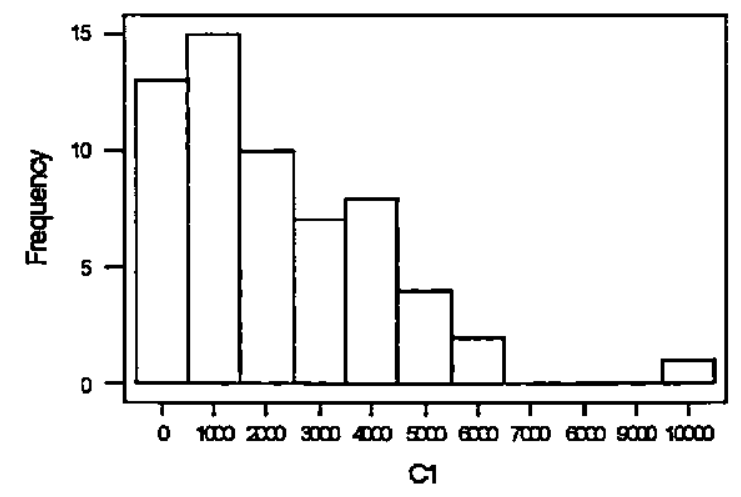

Figura 4.1 : Histograma dos tempos de falhas de materiais elétricos.

Podemos observar pela Figura 4.1 que podemos assumir uma mistura de distribuições para estes dados. Ajustamos misturas de 2 e 3 distribuiçōes Weibull e também mistura de distribuiçōes 2 Gamas Generalizadas.

Os resultados das estimativas clássicas e Bayesianas e seus respectivos intervalos de confiança e credibilidade se encontram na Tabela 4.1. 
Tabela 4.1 Estimativas para os parâmetros.

\begin{tabular}{c|c|c|c|c|c}
\hline Misturas & Parâmetro & EMV & $\begin{array}{c}\text { Intervalo de Confiança } \\
(95 \%)\end{array}$ & $\begin{array}{c}\text { Média a } \\
\text { Posteironi }\end{array}$ & $\begin{array}{c}\text { Intervalo de Credibilidade } \\
(95 \%)\end{array}$ \\
\hline \multirow{4}{*}{$\begin{array}{c}\text { Duas } \\
\text { Weibull }\end{array}$} & $\alpha 01$ & 5,679 & $(2,327 ; 9,030)$ & 5,621 & $(5,046 ; 6,0794)$ \\
\cline { 2 - 6 } & $\alpha 02$ & 19,893 & $(-1,474 ; 41,254)$ & 19,017 & $(18,066 ; 19,939)$ \\
\cline { 2 - 6 } & $\beta 1$ & 0,785 & $(0,393 ; 1,177)$ & 0,781 & $(0,683 ; 0,889)$ \\
\cline { 2 - 6 } & $\beta 2$ & 2,437 & $(-0,052 ; 4,926)$ & 2,332 & $(2,187 ; 2,475)$ \\
\cline { 2 - 6 } & $\mathrm{p}$ & 0,589 & $(0.227 ; 0.932)$ & 0,595 & $(0,348 ; 0,867)$ \\
\hline \multirow{4}{*}{$\begin{array}{c}\text { Três } \\
\text { Weibull }\end{array}$} & $\alpha 01$ & 5,0640 & $(2,692 ; 7,435)$ & 5,562 & $(4,423 ; 6,084)$ \\
\cline { 2 - 6 } & $\alpha 02$ & 50,5321 & $(-24,18 ; 125,24)$ & 50,00 & $(48,063 ; 51,938)$ \\
\cline { 2 - 6 } & $\alpha 03$ & 23,2301 & $(8,628 ; 37,832)$ & 20,473 & $(20,013 ; 21,571)$ \\
\cline { 2 - 6 } & $\beta 1$ & 0,7143 & $(0,439 ; 0,988)$ & 0,7882 & $(0,617 ; 1,185)$ \\
\cline { 2 - 6 } & $\beta 2$ & 7,0999 & $(-3,523 ; 17,723)$ & 7,174 & $(6,064 ; 8,693)$ \\
\cline { 2 - 6 } & $\beta 3$ & 2,8250 & $(1,119 ; 4,530)$ & 3,016 & $(2,064 ; 4,808)$ \\
\cline { 2 - 6 } & $\mathrm{p} 1$ & 0,4900 & $(0,196 ; 0,784)$ & 0,491 & $(0,369 ; 0,613)$ \\
\cline { 2 - 6 } & $\mathrm{p} 2$ & 0,1000 & $(0,0,23)$ & 0,112 & $(0,046 ; 0,201)$ \\
\hline \multirow{4}{*}{$\begin{array}{c}\text { Duas } \\
\text { Gamas } \\
\text { Galiiza- }\end{array}$} & $\alpha 01$ & 7,6052 & $(4,645 ; 10,564)$ & 8,740 & $(7,132 ; 10,323)$ \\
\cline { 2 - 6 } & $\alpha 02$ & 4,6263 & $(1,014 ; 8,023)$ & 4,498 & $(3,962 ; 6,517)$ \\
\cline { 2 - 6 } & $\beta 1$ & 1,1796 & $(-0,472 ; 2,831)$ & 1,202 & $(0,265 ; 2,196)$ \\
\cline { 2 - 6 } & $\beta 2$ & 1,7256 & $(-3,810 ; 7,261)$ & 1,615 & $(0.554 ; 2,873)$ \\
\cline { 2 - 6 } & $\mathrm{k} 1$ & 1,3916 & $(-2,283 ; 5,066)$ & 1,352 & $(0,338 ; 2,452)$ \\
\cline { 2 - 6 } & $\mathrm{k} 2$ & 0,9361 & $(-3,677 ; 5,549)$ & 0,922 & $(0,175 ; 1,764)$ \\
\cline { 2 - 6 } & $\mathrm{p}$ & 0,8559 & $(0,744 ; 0,967)$ & 0,8601 & $(0,751 ; 0,923)$ \\
\hline
\end{tabular}

Tabela 4.2 Valores obtidos no criterio de convergência de Geweke para os parâmetros da mistura de duas Weibull.

\begin{tabular}{c|c}
\hline Parâmetro & Geweke \\
\hline$\alpha 01$ & $-0,025$ \\
\hline$\alpha 02$ & 0,1048 \\
\hline$\beta 1$ & $-0,0228$ \\
\hline$\beta 2$ & 0,0756 \\
\hline$p$ & 0,1206 \\
\hline
\end{tabular}


Tabela 4.3 Valores obtidos no critério de convergência de Geweke para os parâmetros da mistura de três Weibull.

\begin{tabular}{c|c}
\hline Parâmetro & Geweke \\
\hline$\alpha 01$ & 0,0321 \\
\hline$\alpha 02$ & $-0,0670$ \\
\hline$\alpha 03$ & 0,0252 \\
\hline$\beta 1$ & 0,0402 \\
\hline$\beta 2$ & 0,3979 \\
\hline$\beta 3$ & $-0,0868$ \\
\hline$p 1$ & 0,0387 \\
\hline$p 2$ & $-0,0480$ \\
\hline
\end{tabular}

Tabela 4.4 Valores obtidos no critério de convergência de Geweke para os parâmetros da mistura de duas Gamas Generalizadas.

\begin{tabular}{c|c}
\hline Parâmetro & Geweke \\
\hline$\alpha 1$ & 0,015 \\
\hline$\alpha 2$ & 0,2069 \\
\hline$\beta 1$ & $-0,2521$ \\
\hline$\beta 2$ & $-0,9806$ \\
\hline$\kappa 1$ & 0,4270 \\
\hline$\kappa 2$ & 0,5759 \\
\hline $\mathrm{p} 1$ & 0,1709 \\
\hline
\end{tabular}

Pela Tabelas 4.2, 4.3 e 4.4 verificamos a convergência de todos os parâmetros em todos os modelos de mistura ajustados para esses dados, já que temos todos os valores contidos no intervalo [-2;2].

$\mathrm{Na}$ Figura 4.2 abaixo, temos as figuras referentes à mistura de 2 distribuições Weibull .
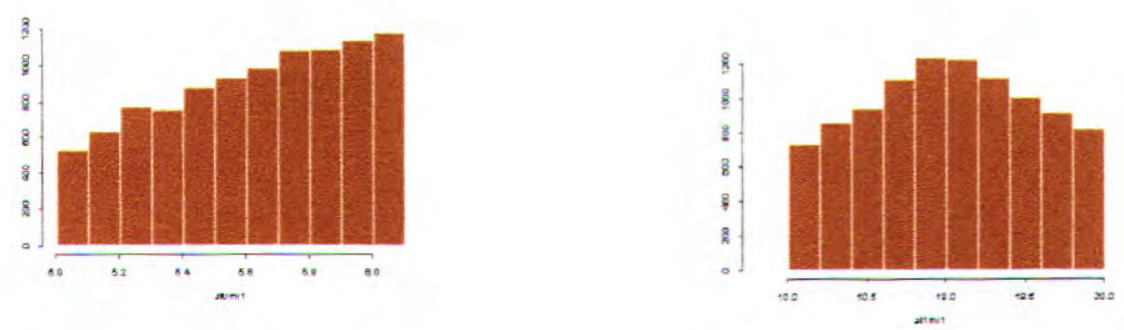

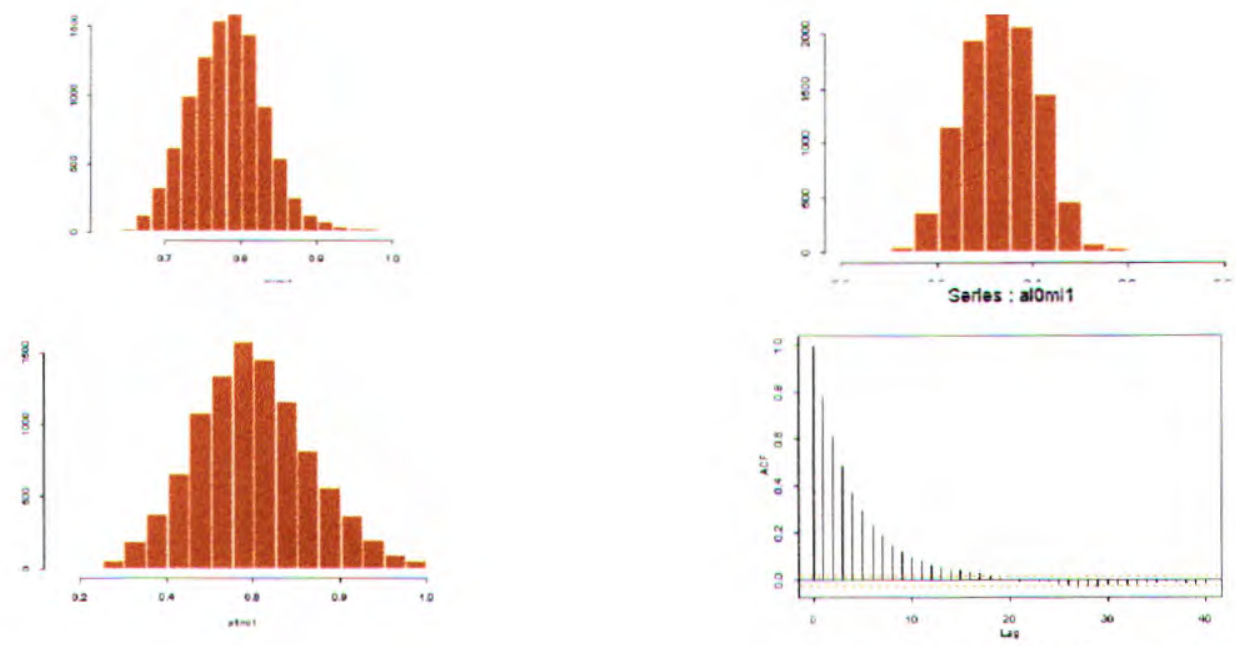

Series : b0mi1
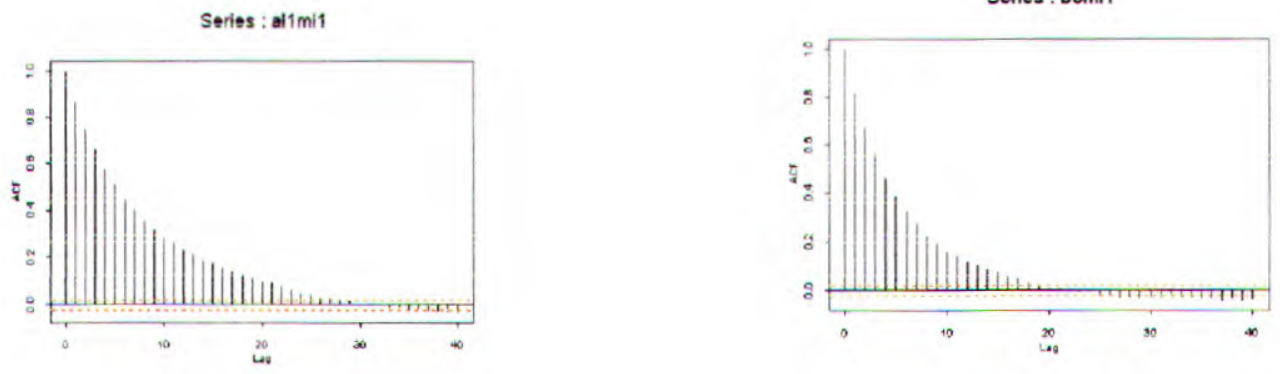

Serles : p1mi1

Serles : biml1
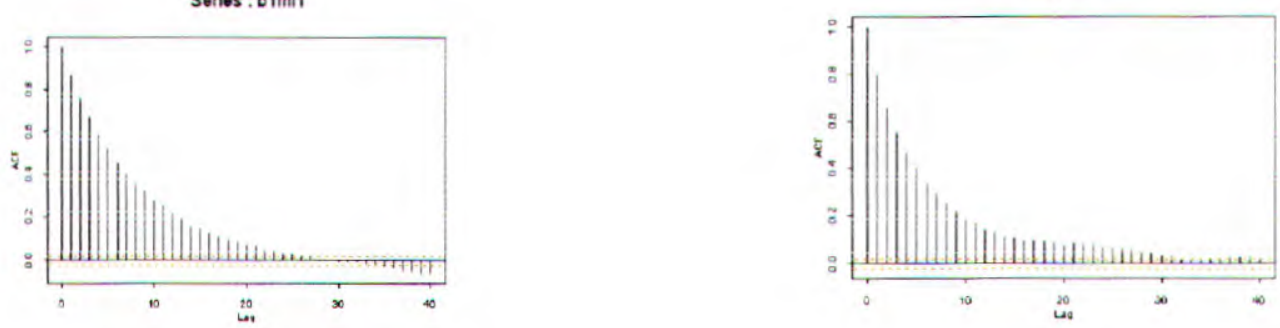

Figura 4.2: Gráficos para o modelo de mistura de 2 Weibull.

Podemos observar pela Figura 4.2 que os gráficos de correlação para todos os parâmetros tiveram uma correlação alta. Com isso, tivemos que selecionar os valores da amostra de $30 \mathrm{em} 30$. Assim, de 11000 valores da cadeia, desprezamos os primeiros 1000 e selecionamos os 10000 restantes de $30 \mathrm{em} \mathrm{30}$, resultando uma amostra final de 333 valores. 
Agora temos os gráficos para o modelo de mistura de 3 Weibull.
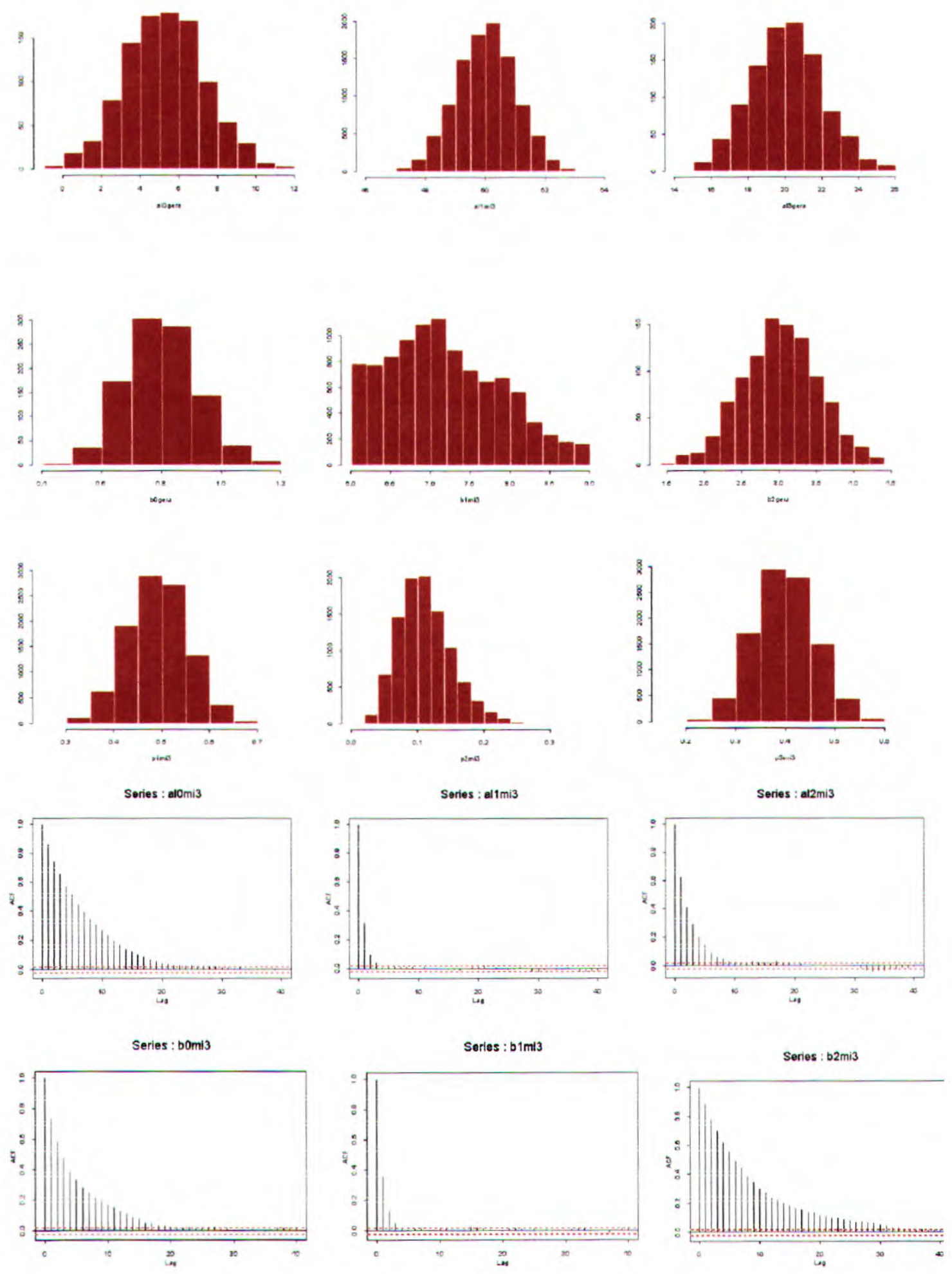
Series : p1mi3

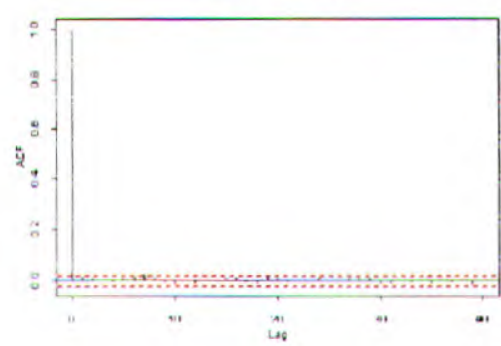

Series : $p 2 m i 3$

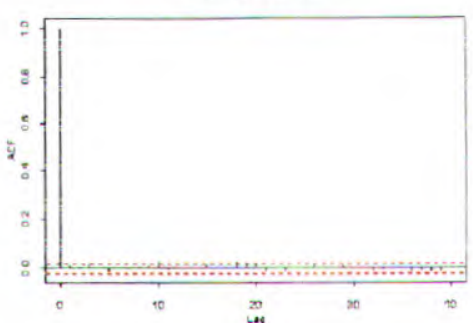

Serles : p3mi3

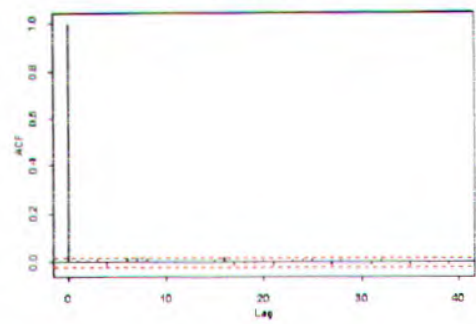

Figura 4.3: Gráficos dos parâmetros para o modelo de mistura de 3 Weibull.

Novamente observamos uma alta correlação entre os valores e a seleção foi obtida com valores de 30 em 30 .

$\mathrm{Na}$ Figura 4.4 temos os gráficos das distribuições a posteriori marginais para os parâmetros do último modelo (mistura de Gamas Generalizadas),
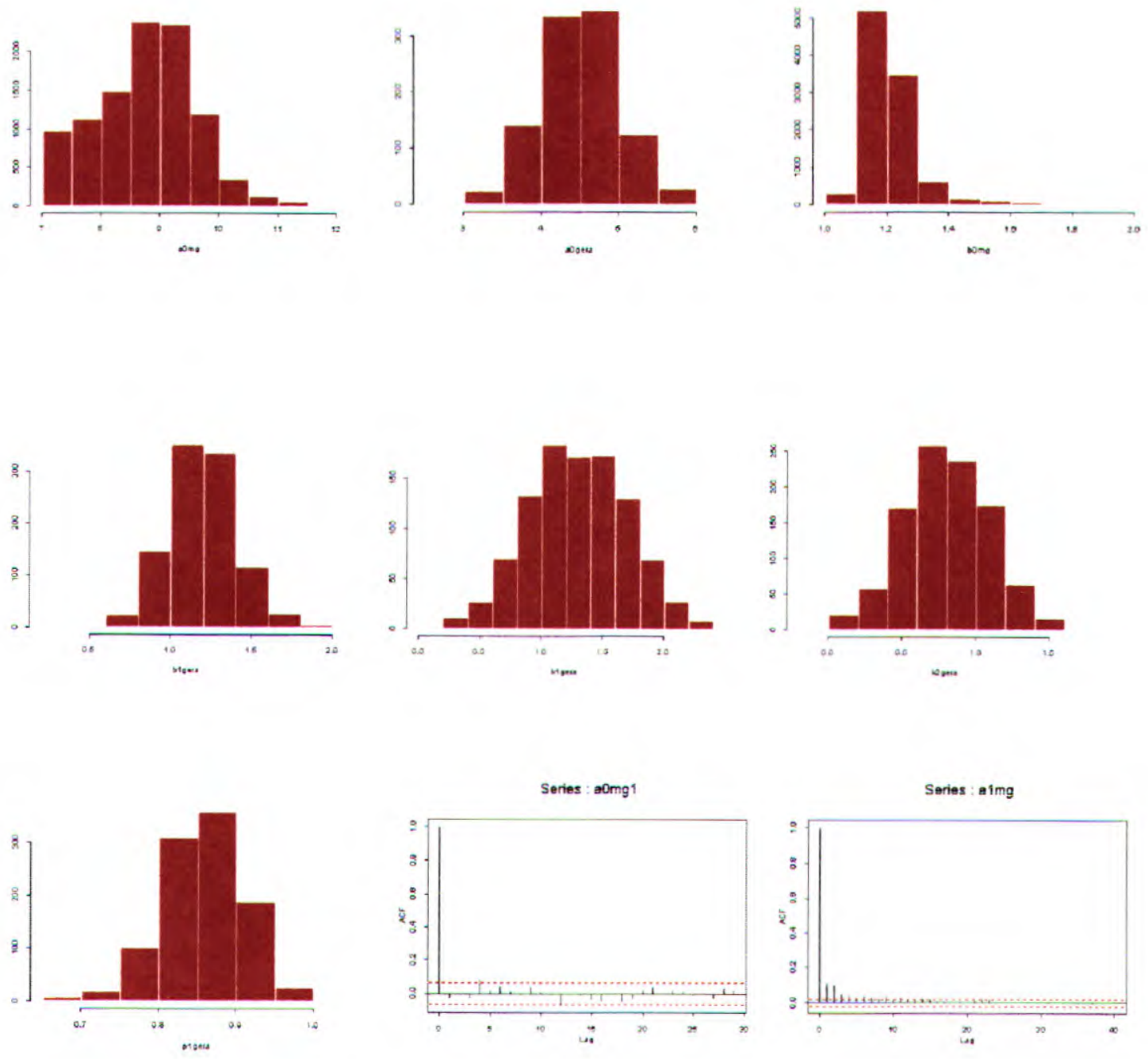
Setles : bohag1

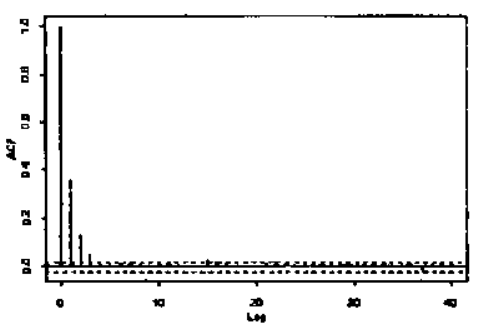

Sorles : $k 2$

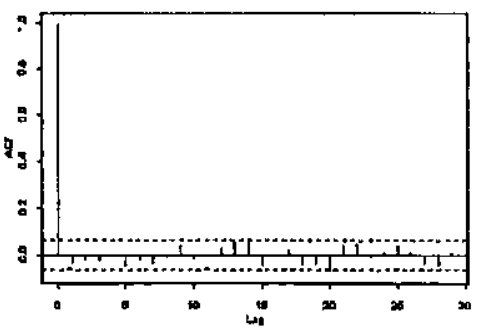

Garks : bimg

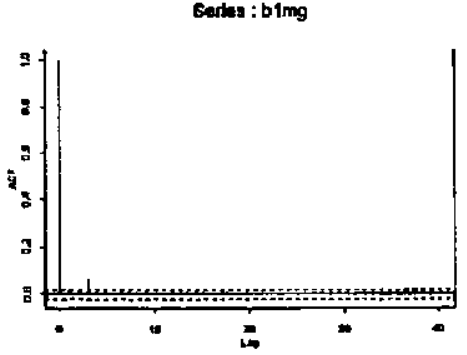

Sotles : p1

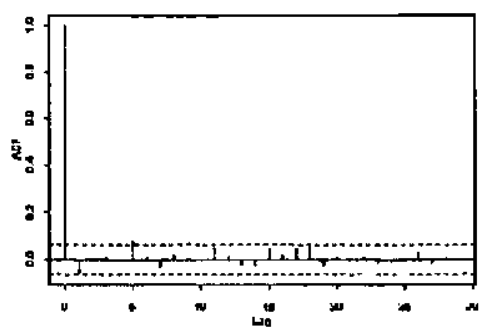

Sortas : $k 1$

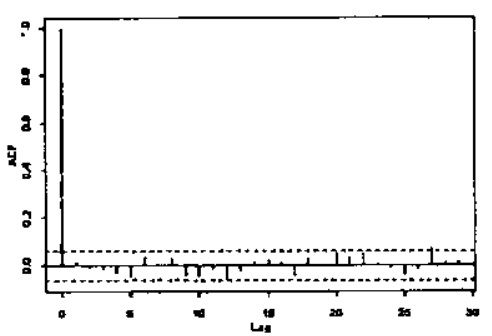

Figura 4.4: Gráficos dos paråmetros para o modelo de 2 Gamas.

A seleção de amostras foi feita escolhendo valores de 5 em 5 já que neste caso não tivemos altas correlaçōes como anteriormente. Assim, tivemos uma amostra final de 2000 valores.

\subsubsection{Modelo de Mistura para os dados 3}

Um histograma dos tempos de falhas de materiais são dados na figura 4.5. Neste caso podemos notar que um modelo de mistura de distribuições parece ser adequado para este conjunto de dados.

Histograma dos Tempos de Falha (Materiais Elétricos)

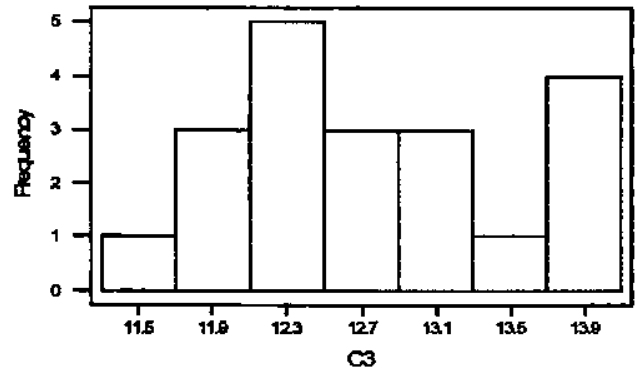

Figura 4.5: Histograma dos tempos de falha de materiais elétricos.

Um primeiro modelo ajustado é o de mistura de normais; um segundo modelo, uma mistura de normal e exponencial. 
Tabela 4.5 Estimativas para os parâmetros.

\begin{tabular}{c|c|c|c|c|c}
\hline \multirow{3}{*}{$\begin{array}{c}\text { Misturas } \\
\text { Duas }\end{array}$} & Parâmetro & EMV & $\begin{array}{c}\text { Intervalo de Confiança } \\
(95 \%)\end{array}$ & $\begin{array}{c}\text { Média a } \\
\text { Posteirori }\end{array}$ & $\begin{array}{c}\text { Intervalo de Credibilidade } \\
(95 \%)\end{array}$ \\
\cline { 2 - 6 } Normais & $\mu_{1}$ & 12,278 & $(12,036 ; 12,520)$ & 12,32 & $(11,96 ; 12,81)$ \\
\cline { 2 - 6 } & $\mu_{2}$ & 13,516 & $(13,203 ; 13,828)$ & 13,47 & $(12,68 ; 14,1)$ \\
\cline { 2 - 6 } & $\sigma$ & 0,346 & $(0,218 ; 0,473)$ & 0,468 & $(0,278 ; 0,868)$ \\
\hline \multirow{4}{*}{\begin{tabular}{c} 
Normal \\
\cline { 2 - 6 }
\end{tabular}} & $\boldsymbol{p}$ & 0,515 & $(-0,545 ; 1,575)$ & 0,6 & $(0,0645 ; 0,814)$ \\
\cline { 2 - 6 } & $\sigma$ & 12,701 & $(12,344 ; 13,057)$ & 12,363 & $(11,071 ; 12,979)$ \\
\cline { 2 - 6 } & $\beta$ & 13,2955 & $(11,659 ; 14,931)$ & 13,128 & $(13,052 ; 13,341)$ \\
\hline & $p$ & 1,000 & - & 0,807 & $(0,638 ; 0,93095)$ \\
\hline
\end{tabular}

Aqui não conseguimos encontrar o valor da estimativa para o parâmetro $p$ do modelo normal- exponencial para o caso clássico. As estimativas eram valores muito grandes (>1) e depois de colocarmos a restrição do parâmetro estar entre 0 e 1,0 valor estimado era sempre o limite superior, ou seja, 1.

No caso de mistura de duas distribuições Normais, temos que a estimativa para o valor $\left|\mu_{1}-\mu_{2}\right| / \sigma$ é dada por 3.867>2. Com isso, temos uma maior evidência a favor desse tipo de modelagem para esses dados.

Além disso, na estimativa clássica para o primeiro modelo, temos um intervalo de confiança assintótico que possui valores negativos para o parâmetro $p$, o que não pode acontecer já que temos uma probabilidade, um valor entre 0 e 1 .

Tabela 4.6 Valores obtidos para $\sqrt{R}$ no critério de convergência de Gelman e Rubin para o parâmetro.

\begin{tabular}{c|c}
\hline & $\sqrt{R}$ \\
\hline$\mu_{1}$ & 1,000 \\
\hline$\mu_{2}$ & 1,000 \\
\hline$\sigma$ & 1,000 \\
\hline$p$ & 1,000 \\
\hline
\end{tabular}


Tabela 4.7 Valores obtidos no critério de convergência de Geweke para os parâmetros da mistura Normal Exponencial.

\begin{tabular}{c|c}
\hline & Geweke \\
\hline$\mu$ & 0,0613 \\
\hline$\sigma$ & $-0,0579$ \\
\hline$\beta$ & $-0,0237$ \\
\hline$p$ & $-0,0483$ \\
\hline
\end{tabular}

Para este conjunto de dados também observamos convergência para os dois modelos de mistura ajustados. A diferença dos critérios se dá devido ao uso de diferentes softwares utilizados para obter as estimativas Bayesianas. Para a mistura de Normais, utilizamos o WinBugs e verificamos a convergência pelo Gelman e Rubin, para a mistura Normal - Exponencial utilizamos o Ox e com isso verificamos a convergência através do Geweke, no Matllab.

Nas figuras abaixo, temos os gráficos de convergência, correlação e histograma dos parâmetros.
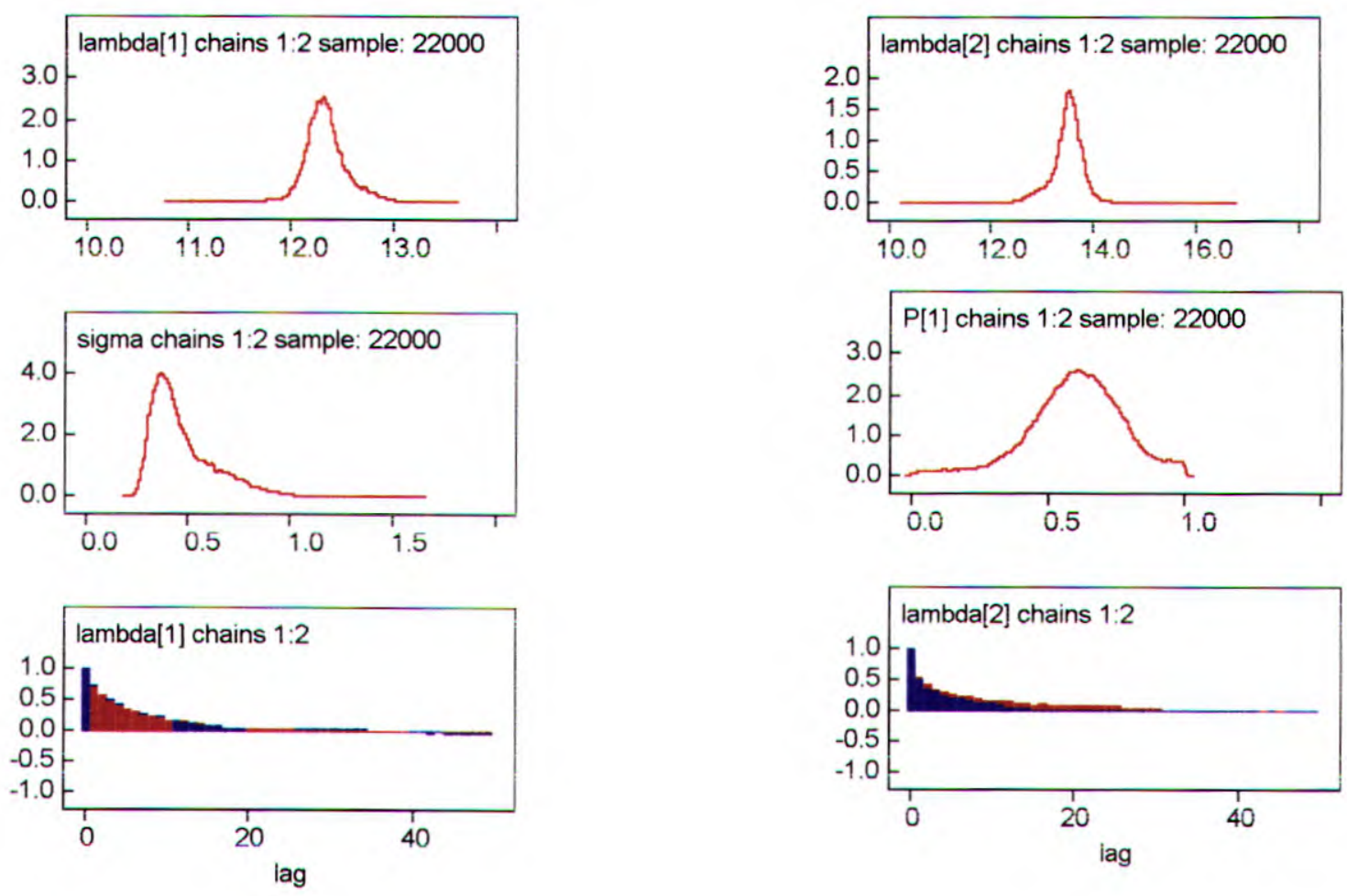

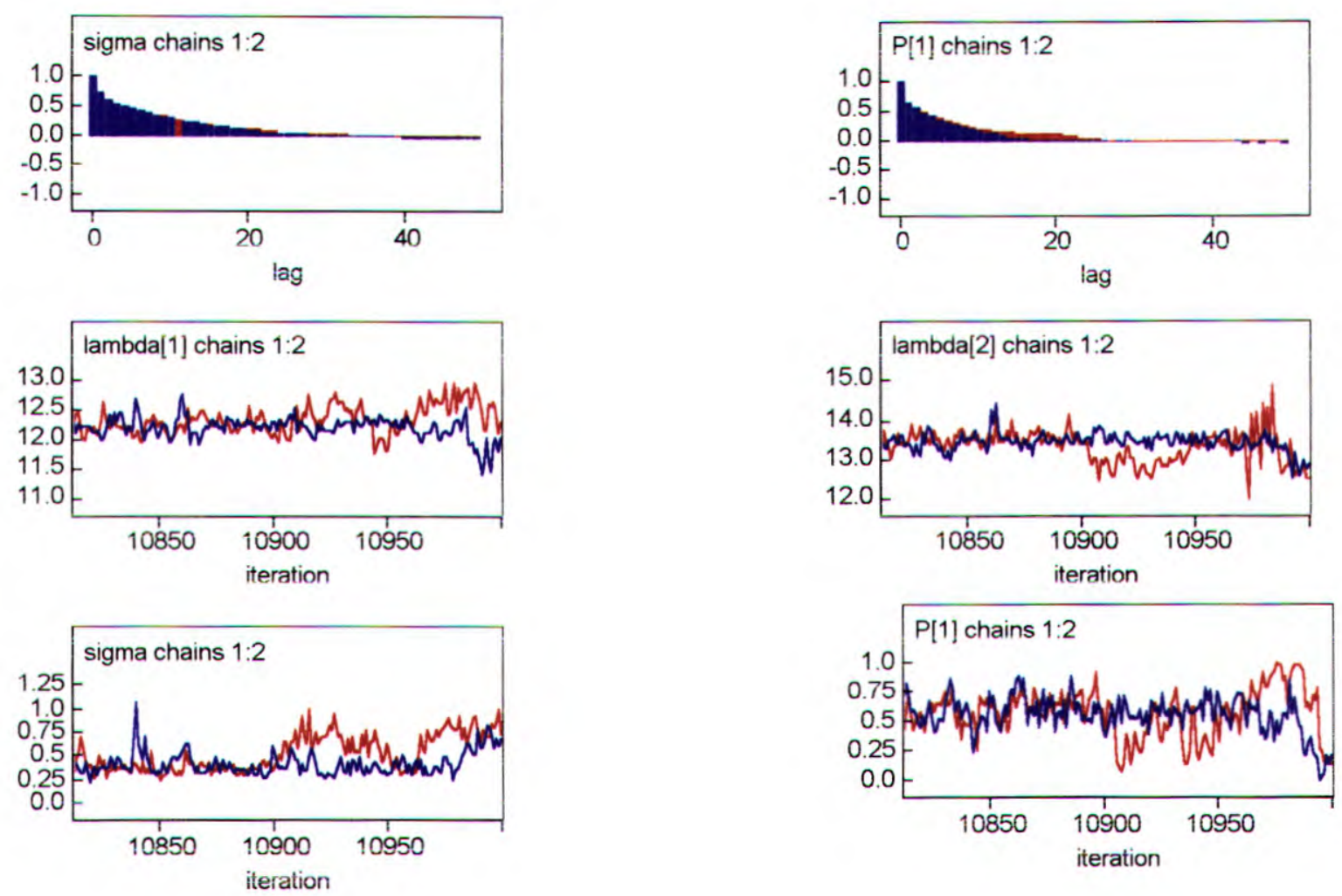

Figura 4.4 : Gráficos do modelo de mistura de normais.

Geramos duas cadeias com 11000 iterações cada e desprezamos os primeiros 1000 valores. Tivemos um correlação alta e selecionamos os valores de $20 \mathrm{em} \mathrm{20,}$ totalizando uma amostra de 1000 valores.

Abaixo temos as figuras referentes ao outro modelo de mistura, ou seja, a mistura normal- exponencial. Como essas estimativas foram feitas no Ox, temos os gráficos em S-Plus.
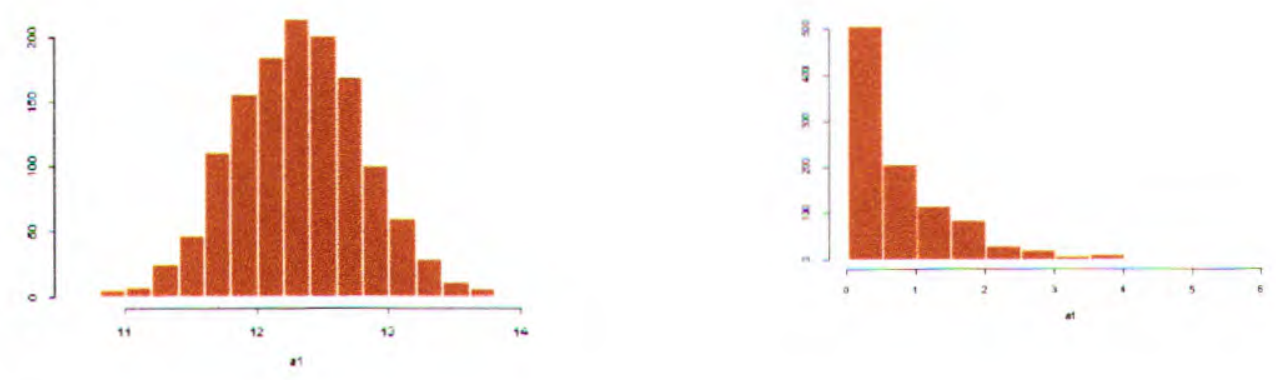


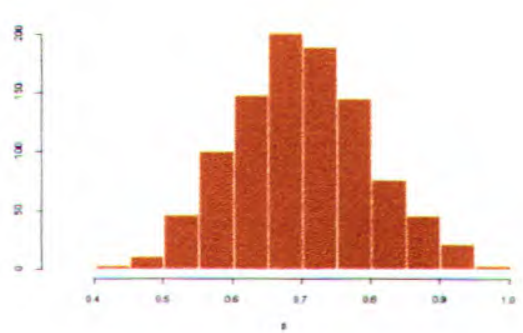

Series : mine

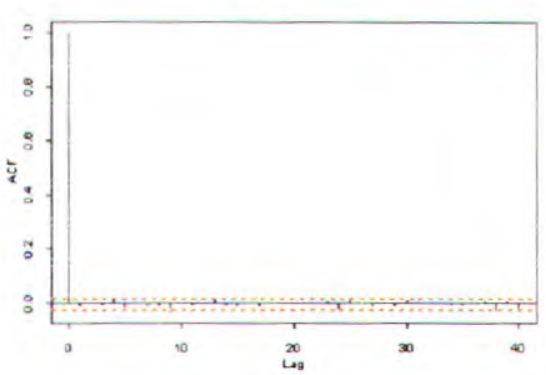

Series : betne

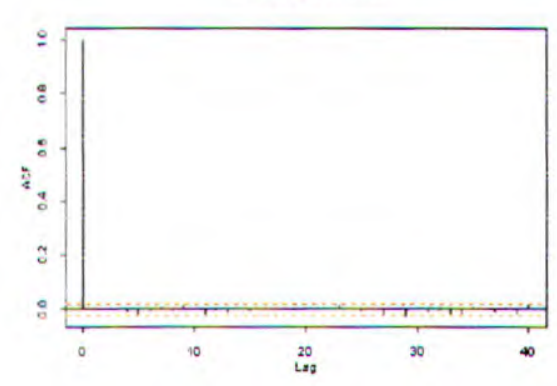

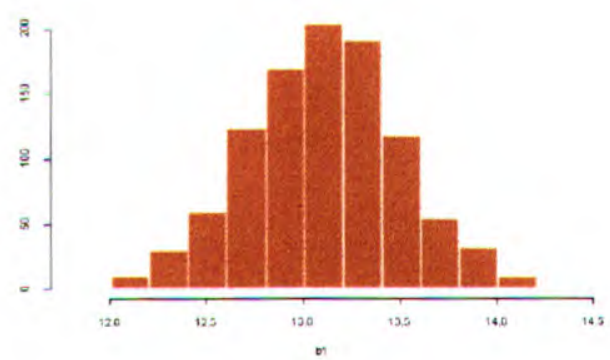

Series : signe

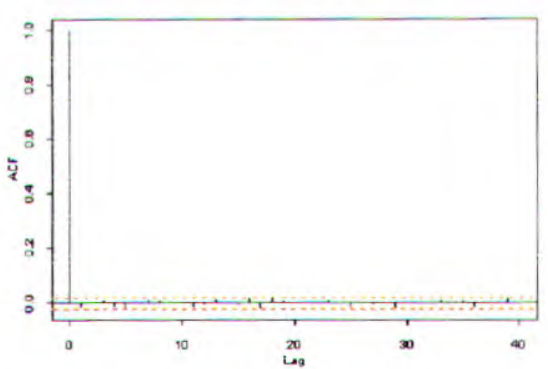

Series : p1ne

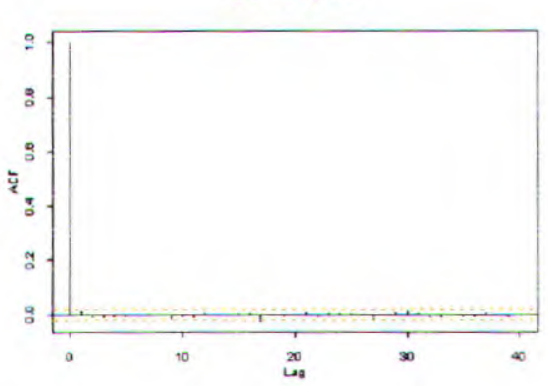

Figura 4.5 : Gráficos da mistura normal- exponencial.

Agora geramos uma cadeia com 11000 iterações cada e desprezamos os primeiros 1000 valores. Para todos os parâmetros deste modelo a correlação foi pequena e por isso selecionamos os valores de $2 \mathrm{em} \mathrm{2,} \mathrm{totalizando} \mathrm{uma} \mathrm{amostra} \mathrm{de}$ 5000 valores.

Neste capítulo podemos notar que os modelos de misturas são uma alternativa aos modelos ajustados anteriormente. Podemos notar que para alguns casos, existe uma bi ou trimodalidade nos dados já nos dando um indício que um modelo mais flexível dever ser utilizado. No próximo Capítulo selecionamos os modelos ajustados e daí comparamos os modelos de mistura com os ajustados anteriormente. 


\section{CAPÍTULO 5: Comparação e Seleção de Modelos}

Nos Capítulos anteriores fizemos análises clássicas e Bayesianas para aiguns conjuntos de dados e vários modelos foram ajustados para cada um. Agora, precisamos decidir dentre todos os modelos ajustados qual é o que melhor se ajusta aos dados. Assim, neste Capítulo estamos interessados em selecionar os modelos vistos nos Capítulos 3 e 4 .

Para isto, vamos utilizar um critério de seleção clássico, dado pelo Akaike ( AIC )e um Bayesiano, pelo critério da densidade preditiva ordenada.

\subsection{Critério Clássico de seleção de modelos}

Uma forma de selecionar modelos é o uso do critério de mínima informação conhecida como AlC, proposta por Akaike (ver Box et al. 1994). Esses modelos são muito utilizados em séries temporais mas com alguns ajustes podem ser utilizados na seleção de modelos de sobrevivência (ver por exemplo, Klein \& Moeschberger 1997 ).

Para modelos de sénies temporais temos,

$$
A I C_{p, q}=\frac{-2(l)+2 r}{n}
$$

onde $l$ denota o logaritmo da função de verossimilhança maximizada, $r=p+q+1$ denota o número de parâmetros estimados no modelo, incluindo um termo constante e $n$ denota o tamanho da série. $O$ segundo termo seria um 'fator de penalidade' para a inclusão de parâmetros adicionais no modelo. No critério de mínima informação, modelos com menores $A I C$ são prefenidos.

Para o nosso caso, utilizamos esse critério sem a divisão por $n$, ou seja,

$$
A I C=-2(l)+2(p)
$$

onde $p$ denota o número de paråmetros estimados pelo modelo. 
É interessante observar que esse critério é extremamente fácil de ser utilizado, pois o 'software' SAS nos fornece o logaritmo da função de verossimilhança na análise Clássica dos modelos.

\subsection{Critério Bayesiano de seleção de modelos}

Para um conjunto de dados podemos ter interesse em discriminar dois ou mais modelos. Uma forma alternativa é dada pelo uso de densidades preditivas ordenadas (CPO) para selecionar o melhor modelo. A densidade preditiva ordenada para uma obsevação $t_{i}$ é dada por,

$c_{i}=f\left(t_{i} \mid t_{(i)}, x_{i}\right)=\int f\left(t_{i} \mid \theta, x_{i}\right) \pi\left(\theta \mid t_{(i)}, x_{(i)}\right) d \theta$

onde $\pi\left(\theta \mid t_{(i)}, x_{i}\right)$ é a densidade a posteriori de $\theta$ dado $t_{(t)}=\left(t_{1}, \ldots, t_{t-1}, t_{i+1}, \ldots, t_{n}\right)$ e $x_{i}$ é o vetor de covariáveis associado à $t_{(i)}$.

Usando as amostras geradas pelo amostrador de Gibbs, podemos aproximar $f\left(t_{i} \mid t_{(i)}, x_{i}\right)$ pela estimativa de Monte Cario

$\hat{f}\left(t_{i} \mid t_{(i)}, x_{i}\right)=\frac{1}{S} \sum_{r=1}^{S} f\left(t_{i} \mid x_{i}, \theta^{(s)}\right)$

onde S é o número de amostras geradas pelo algoritmo.

Podemos usar $c_{i}$ para selecionar modelos. Deste modo, consideramos gráficos de $c_{i}$ versus $i(i=1,2, \ldots, n)$ para diferentes modelos. Considerando os valores observados, o melhor modelo tem, em geral, maiores valores de $c_{i}$.

Além disso, podemos escolher o modelo cujo produto de $c_{i}$ seja máximo, ou seja, $\quad c(l)=\prod c_{i}(l)$, onde $l$ indexa modelos. 


\subsection{Seleção para o primeiro conjunto de dados}

O primeiro conjunto de dados que utilizamos foi o de 17 pacientes com leucemia. (Feigl e Zelen, 1965)

Para este conjunto de dados ajustados os seguintes modelos: Exponencial, Weibull, Logístico e Log-normal.

Abaixo, temos os gráficos de CPO para os vários modelos ajustados. $\mathrm{Na}$ legenda sempre teremos CPO acrescido de alguma letra que indica o modelo, por exemplo, CPOE indica a CPO do modelo Exponencial (E), CPOW (modelo Weibull) e assim sucessivamente.

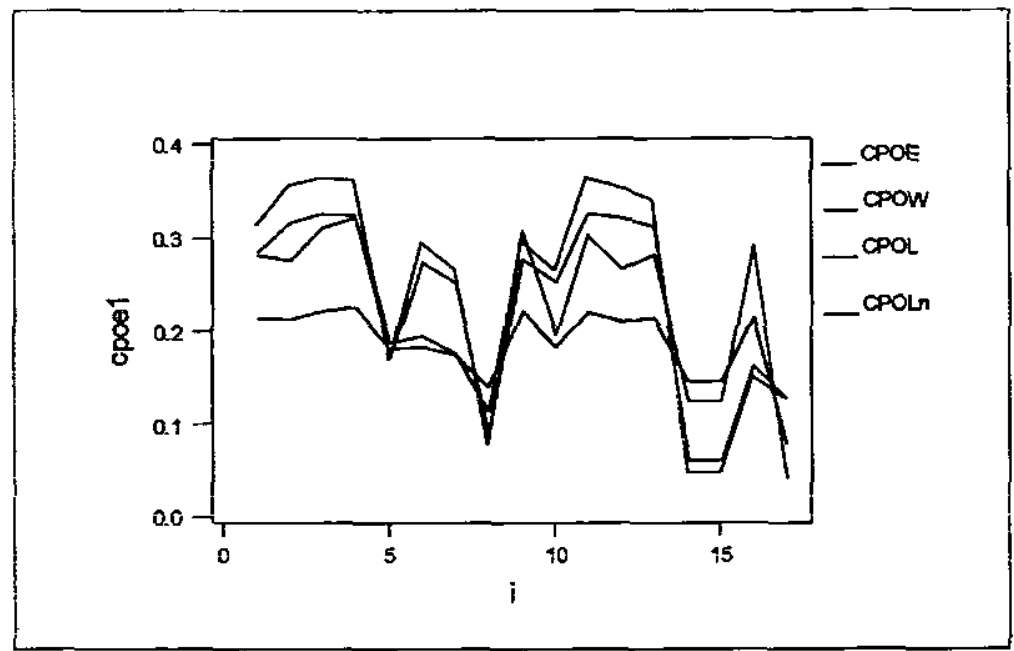

Figura 5.1: Gráfico de CPO para os modelos dos dados 1.

Além do gráfico, temos na Tabela 5.1 os valores AlC e o produto de $c_{i}$ para cada modelo.

Tabela 5.1: Mínima informação de Akaike e produto de CPO para os dados1.

\begin{tabular}{c|c|c}
\hline Modelo & AIC & $\mathrm{c}(\mathbf{l})$ \\
\hline Exponencial & $\mathbf{5 8 , 4 1 5}$ & $\mathbf{c}(\mathbf{1})=\mathbf{2 , 4 5 e - 1 2}$ \\
\hline Weibull & 60,403 & $\mathrm{c}(2)=1,95 \mathrm{e}-12$ \\
\hline Logístico & 61,049 & $\mathrm{c}(3)=1,58 \mathrm{e}-12$ \\
\hline Lognormal & 60,154 & $\mathrm{c}(4)=2,96 \mathrm{e}-13$ \\
\hline
\end{tabular}


Para esse conjunto de dados, tirariamos a mesma conclusão com qualquer tipo de seleção de modelos. De qualquer maneira, o modelo Exponencial foi o que melhor se ajustou aos dados.

\subsection{Seleção para o segundo conjunto de dados}

O segundo conjunto de dados utilizado foi o de Lawless(1982) sobre tempos de falha.

Para este conjunto de dados ajustados os seguintes modelos: Exponencial, Weibull, Logístico e Log-normal, Gama Generalizada, Mistura de 2 e 3 distribuições Weibull e Mistura de 2 distribuições Gamas Generalizadas.

A Figura 5.2 abaixo nos mostra os valores da CPO para os 7 modelos ajustados para esse conjunto de dados. A legenda foi feita da mesma maneira que a figura anterior, lembrando que agora temos modelos de mistura que foram assim colocados CPOM2w: mistura de 2 Weibull, CPOM3w: mistura de 3 Weibull e CPOMgg : mistura de 2 Gamas Generalizadas.

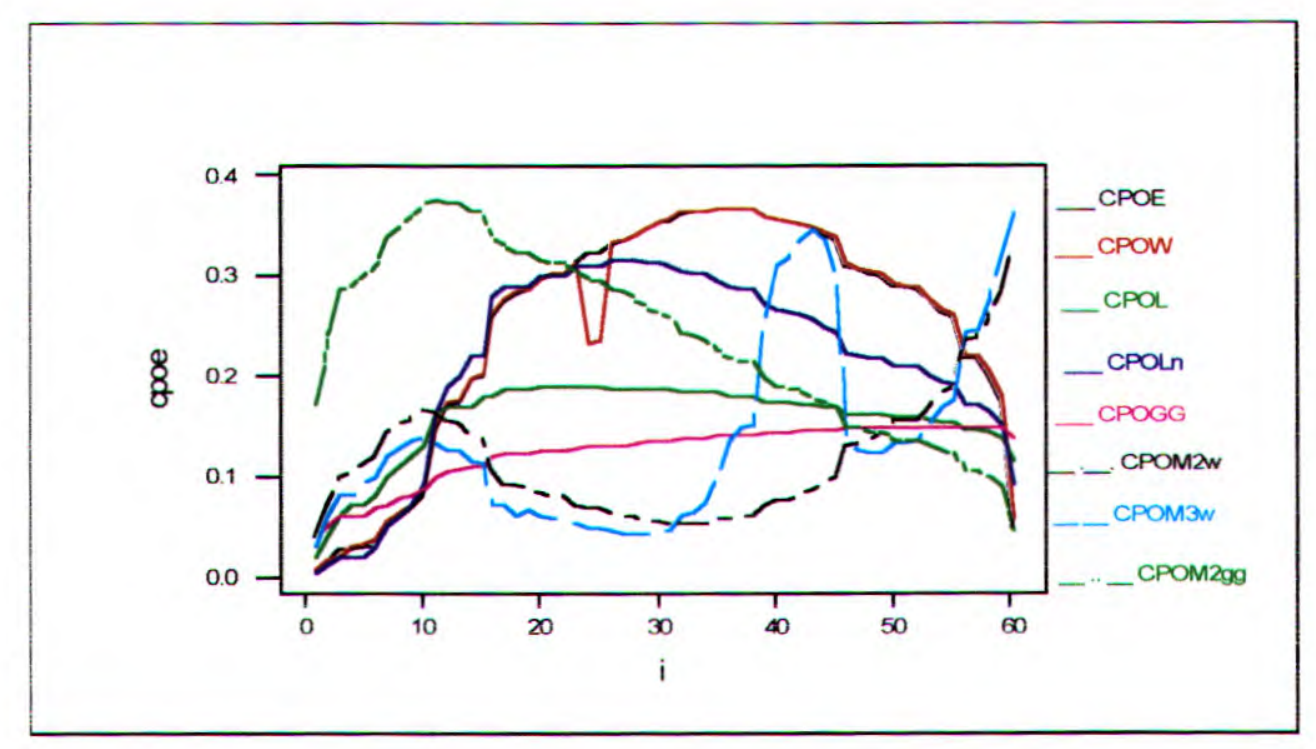

Figura 5.2: Gráfico de CPO para os modelos dos dados 2. 
Tabela 5.2: Minima informação de Akaike e produto de CPO para os dados2.

\begin{tabular}{c|c|c}
\hline Modelo & AIC & $\mathrm{c}(\mathrm{l})$ \\
\hline Exponencial & 1048,2 & $\mathrm{c}(1)=4,37 \mathrm{e}-43$ \\
\hline Weibull & 1048,16 & $\mathrm{c}(2)=9,41 \mathrm{e}-43$ \\
\hline Logístico & 1051,88 & $\mathrm{c}(3)=3,59 \mathrm{e}-50$ \\
\hline Lognormal & 1049,72 & $\mathrm{c}(4)=1,32 \mathrm{e}-46$ \\
\hline Gama Generalizada & $\mathbf{1 0 4 6 , 1 4}$ & $\mathrm{c}(5)=6,85 \mathrm{e}-56$ \\
\hline Mistura 2 Weibull & 1048,52 & $\mathrm{c}(6)=6,31 \mathrm{e}-60$ \\
\hline Mistura 3 Weibull & 1051,62 & $\mathrm{c}(7)=7,08 \mathrm{e}-58$ \\
\hline Mistura 2 Gamas & 1048,02 & $\mathbf{c ( 8 )}=\mathbf{3 , 5 7 e - 4 0}$ \\
\hline
\end{tabular}

Para o segundo conjunto de dados já temos que selecionar o melhor modelo com mais cuidado. Utilizando o critério clássico, escolheriamos o modelo Gama Generalizada. No entanto, utilizando a seleção Bayesiana, a mistura de Gamas Generalizadas seria o melhor modelo a ser escolhido. Observando a Figura 5.2, vemos que o modelo de mistura de 2 Gamas foi superior aos outros até a observação 20 e só depois foi menos eficiente. Através da mesma Figura podemos notar que o modelo Gama Generalizada foi mais constante mas em nenhum momento esteve superior aos outros modelos.

\subsection{Seleção para o terceiro conjunto de dados}

Para este conjunto de dados ajustados os seguintes modelos: Exponencial, Weibull, Logistico e Log-normal, Mistura de 2 Normais e Mistura de Normal e Exponencial e Birbaum- Saunders.

Utilizando a mesma lógica para a legenda, temos abaixo a Figura 5.3 que nos mostra os valores da preditiva ordenada ponto a ponto. 


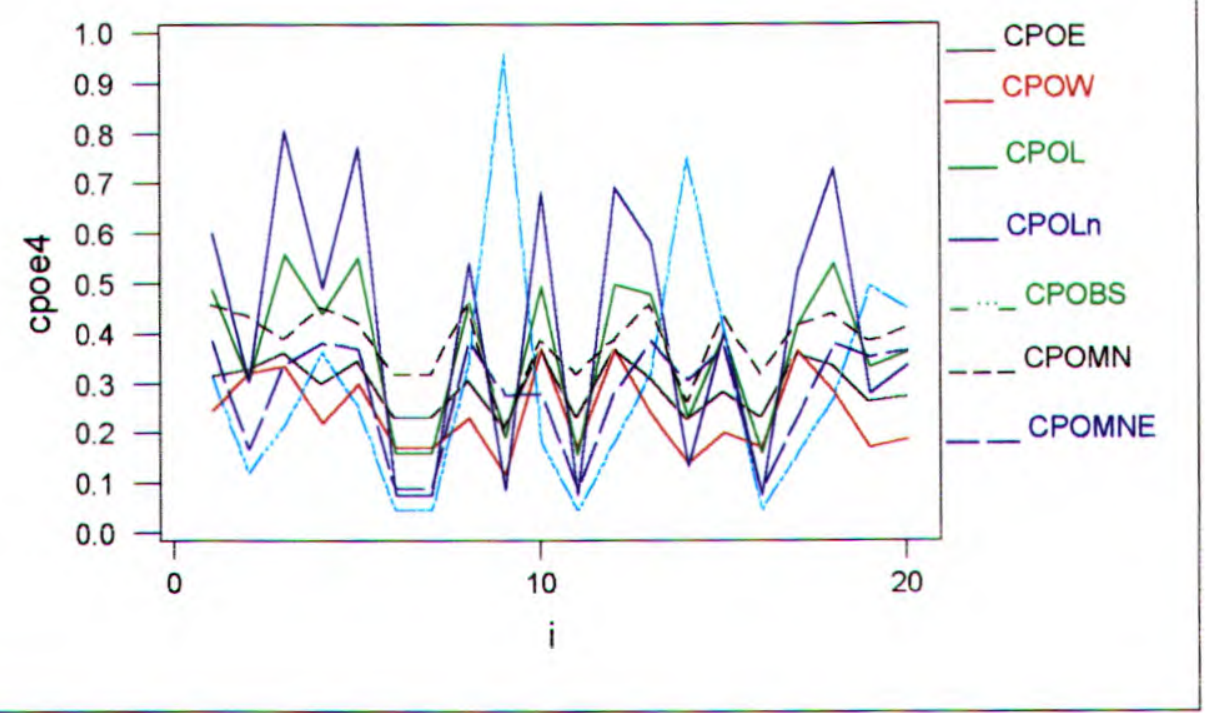

Figura 5.3: Gráfico dc CPO para os modclos dos dados 3.

Tabela 5.3: Mínima informação de Akaike e produto de CPO para os dados3.

\begin{tabular}{c|c|c}
\hline Modelo & AIC & $\mathrm{c}(1)$ \\
\hline Exponencial & 52,78 & $\mathrm{C}(1)=1,53 \mathrm{e}-11$ \\
\hline Weibull & 50,20 & $\mathrm{C}(2)=1,11 \mathrm{e}-13$ \\
\hline Logístico & 48,72 & $\mathrm{C}(3)=3,01 \mathrm{e}-10$ \\
\hline Lognormal & 48,00 & $\mathrm{C}(4)=4,02 \mathrm{e}-11$ \\
\hline Mistura 2 Normais & 45,88 & $\mathrm{C}(5)=3,054 \mathrm{e}-09$ \\
\hline Mistura Normal Exponcial & 41,90 & $\mathrm{C}(6)=6,21 \mathrm{e}-13$ \\
\hline Birbaum- Saunders & 42,40 & $\mathrm{C}(7)=2,32 \mathrm{e}-14$ \\
\hline
\end{tabular}

No terceiro conjunto de dados, tivemos um problema parecido com 0 segundo, ou seja, os critério clássico e Bayesiano selecionam modelos diferentes. Porém, desta vez, ambos escolhem os modelos de mistura como melhores. 0 clássico selecionou a mistura Normal-Exponencial e o Bayesiano a mistura de 2 Normais. 


\subsection{Seleção para o quarto conjunto de dados}

O último conjunto de dados foi o mais complicado de trabalhar, em função de várias covariáveis.

Na Figura 5.4 temos os gráficos de CPO e pela Tabela 5.4 também temos os valores de $\mathrm{AIC}$ e dos produtos da CPO. Em ambos os casos, temos que para este conjunto de dados, o modelo de regressão Gama Generalizada foi o melhor.

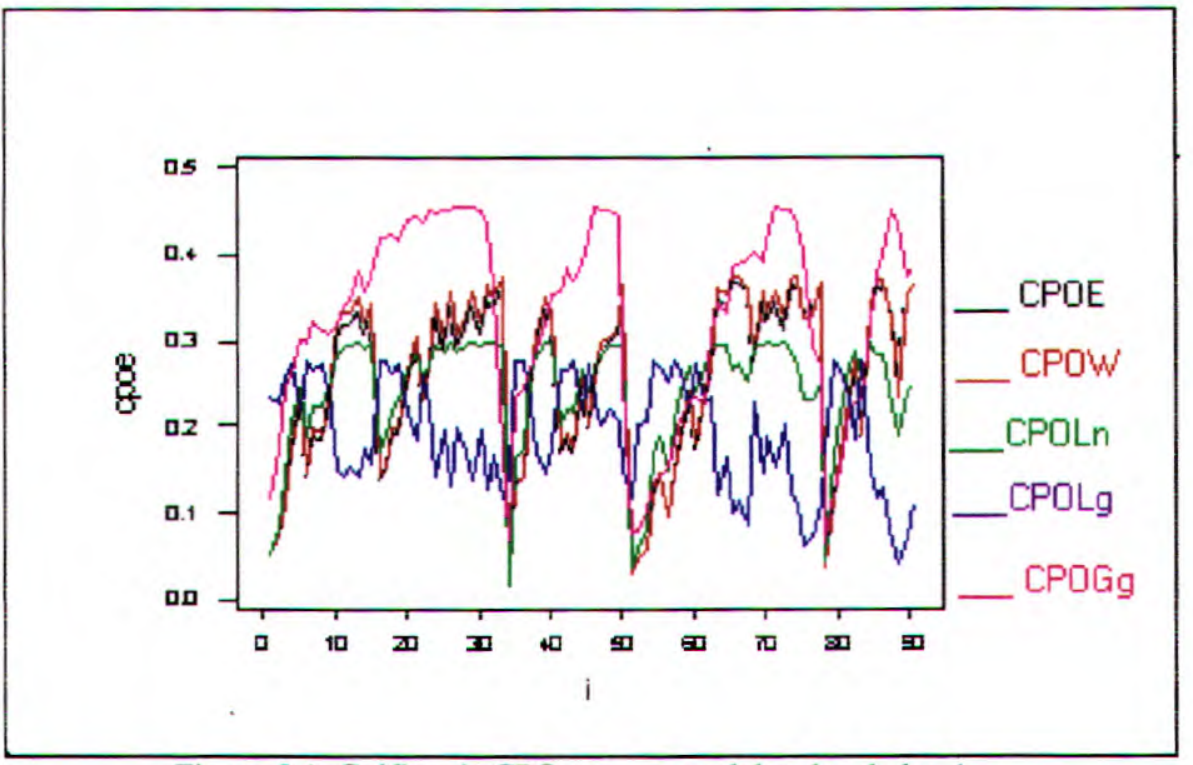

Figura 5.4: Gráfico de CPO para os modelos dos dados 4.

Tabela 5.4: Mínima informação de Akaike e produto de CPO para os dados4.

\begin{tabular}{l|c|c}
\hline Distribuição & AIC & $\mathrm{c}(1)$ \\
\hline Exponencial & 219.05 & $\mathrm{c}(1)=5,904 \mathrm{e}-60$ \\
\hline Weibull & 218.00 & $\mathrm{c}(2)=1,144 \mathrm{e}-58$ \\
\hline Logística & 219.99 & $\mathrm{c}(3)=7,254 \mathrm{e}-60$ \\
\hline Lognormal & 218.99 & $\mathrm{c}(4)=1,057 \mathrm{e}-67$ \\
\hline Gama Generalizada & $\mathbf{2 0 5 . 0 0}$ & $\mathbf{c ( 5 )}=\mathbf{8 , 5 5 2} \mathbf{e}-\mathbf{5 4}$ \\
\hline
\end{tabular}

Com isso, terminamos o Capítulo 5 no qual apresentamos algumas formas de selecionar modelos. Lembramos que essas não são as únicas alternativas de selecionarmos modelos. Acreditamos que a forma Bayesiana de selecionar modelos é mais interessante já que pelos gráficos podemos observar bem em que região um modelo é superior ao outro. Pelo critério clássico, nos baseamos somente em um número e o peso dado a adição de parâmetros é relativamente alto. 


\section{CAPÍTULO 6 : Uso de Métodos Bayesianos em Modelos de Riscos Proporcionais}

Até agora utilizamos modelos log-lineares nos nossos problemas.

Modelos de riscos proporcionais com enfoque Bayesiano serão tratados neste Capítulo.

\subsection{Modelos de Riscos Proporcionais}

No Capítulo 2 apenas mencionamos esse tipo de modelo. Lá, vimos na equação (2.4) que indivíduos diferentes tem funções de risco proporcionais.

Nesta seção também estaremos considerando a distribuição Weibull mas com algumas diferenças da usada na seção 2.1. Assumimos dados com covariáveis e sem censuras. Com isso as funções de risco e Sobrevivência são dadas por:

$$
\begin{aligned}
& h_{0}(t)=\lambda \alpha t^{\alpha-t} \exp (\mathbf{x} \boldsymbol{\beta}) \\
& S(t \mid \mathbf{X})=\exp \left\{-\lambda \exp (\mathbf{x} \boldsymbol{\beta}) t^{\alpha}\right\}
\end{aligned}
$$

Podemos notar que a função densidade de probabilidade está escrita com outra parametrização,

$$
f(t \mid \mathbf{X})=\lambda \alpha t^{\alpha-1} \exp (\mathbf{x} \boldsymbol{\beta}) \exp \left\{-\lambda \exp (\mathbf{x} \boldsymbol{\beta}) t^{\alpha}\right\}
$$

Para dados sem censuras a função de verossimilhança é dada por,

$$
L(\alpha, \lambda, \boldsymbol{\beta})=\lambda^{n} \alpha^{n}\left\{\prod_{i=1}^{n} t_{i}^{\alpha-1}\right\} \exp \left\{\sum_{i=1}^{n} \mathbf{x}_{i} \boldsymbol{\beta}-\lambda \sum_{i=1}^{n} \exp \left(\mathbf{x}_{i} \boldsymbol{\beta}\right) t_{i}^{\alpha}\right\}
$$

As distribuições a priori utilizadas foram:

$$
\begin{aligned}
& \text { (i) } \lambda, \alpha \sim \Gamma(a, b) \text { com a e b conhecidos } \\
& \text { (ii) } \beta \sim N\left(c, d^{2}\right) \text { com ce d conhecidos }
\end{aligned}
$$


Com isso, temos as seguintes distribuições condicionais para o algoritmo ARMS, na forma logaritmica:

$$
\begin{aligned}
& l_{\lambda}=n \log (\lambda)-\lambda \sum_{i=1}^{n} \exp (\mathbf{x} \boldsymbol{\beta}) \mathbf{t}_{\mathrm{i}}^{\alpha}+\log \pi(\lambda) \\
& l_{\alpha}=n \log (\alpha)+(\alpha-1) \sum_{i=1}^{n} \log \left(t_{i}\right)-\lambda \sum_{i=1}^{n} \exp (\mathbf{x} \boldsymbol{\beta}) \mathbf{t}_{\mathrm{i}}^{\alpha}+\log \pi(\alpha) \\
& l_{\beta}=\sum_{i=1}^{n} \mathbf{x} \boldsymbol{\beta}-\lambda \sum_{i=1}^{n} \exp (\mathbf{x} \mathbf{\beta}) \mathfrak{t}_{\mathrm{i}}^{\alpha}+\log \pi(\beta)
\end{aligned}
$$

\subsection{Modelo de Efeitos Aleatórios}

Assumir a função de sobrevivência dada em (6.2), onde incluímos Z, um efeito aleatório com distribuição $N\left(0, \sigma^{2}\right)$.

$$
S(t \mid \mathbf{X})=\exp \left(-\lambda \exp (z+\mathbf{x} \mathbf{\beta}) \mathfrak{t}^{\alpha}\right)
$$

Considerando a transformação abaixo no logaritmo da Função de Sobrevivência, temos:

$$
\begin{aligned}
& \ln \left[-\ln \left(S\left(t_{i} \mid \mathbf{X}\right)\right]=\ln (\lambda)+\alpha \ln \left(t_{i}\right)+z_{i}+\mathbf{x} \boldsymbol{\beta}\right. \\
& \therefore z_{\mathrm{i}}=W\left(x_{i}\right)-\ln (\lambda)-\alpha \ln \left(t_{i}\right)-\mathbf{x} \boldsymbol{\beta}
\end{aligned}
$$

onde $W\left(x_{i}\right)=\ln \left\lfloor-\ln \left(S\left(t_{i}^{*} \mid \mathbf{X}\right)\right)\right\rfloor$ e $\mathrm{t}_{\mathrm{i}}$ é gerado por uma distribuição Weibull com $S(t \mid X)=\exp \left\{-\lambda \exp \left(z+x^{\prime} \beta\right) t^{\alpha}\right\}$ em cada iteração do algoritmo.

A função de Verossimilhança para dados sem censuras é dada por;

$$
L\left(\lambda, \alpha, \boldsymbol{\beta}, \sigma^{2}\right)=\prod_{i=1}^{n} \frac{1}{\sqrt{2 \pi} \sigma} \exp \left\{-\frac{z_{i}^{2}}{2 \sigma^{2}}\right\}
$$

onde $z_{i}$ é dado pela expressão (6.10) acima. 
Considerar as mesmas distribuições a priori do modelo de riscos proporcionais para os parâmetros dos dois modelos e para $\sigma$,

(i) $\sigma^{2} \sim I G(e, f)$ com e, f conhecidos

Os logaritmos das distribuições Condicionais para o algoritmo ARMS são dadas por,

$$
\begin{aligned}
& l_{\lambda}=l_{\alpha}=l_{\beta}=-\frac{1}{2 \sigma^{2}} \sum_{i=1}^{n} z_{i}^{2}+\log \pi(\lambda) \text { ou } \log \pi(\alpha) \text { ou } \log \pi(\beta) \\
& l_{\sigma}=-n \log (\sigma)-\frac{1}{2 \sigma^{2}} \sum_{i=1}^{n} z_{i}^{2}+\log \pi(\sigma)
\end{aligned}
$$

Assim, podemos fazer sem grandes dificuldades um programa no software Ox para encontrar as estimativas Bayesianas para este modelo.

Observar que podemos calcular a previsão de $t_{1}$ por:

$$
\hat{i}_{i}=\exp \left\{\frac{1}{\alpha}\left(W\left(x_{i}\right)-\ln \lambda-x_{i} \beta\right)\right\}
$$

$$
\text { É importante lembrar que } E\left(Z_{i}\right)=0 \text {. }
$$

\subsection{Modelo de Efeitos Aleatórios com Mistura de Normais}

Utilizando o modelo de efeitos aleatórios também poderíamos considerar um modelo de mistura de distribuições para Z em (6.9).

Como anteriormente $Z$ pode ser dado pela fórmula 6.10 e sua verossimilhança com dados ampliados é dada por,

$$
\begin{aligned}
L_{A} & =\prod_{i=1}^{n} \prod_{j=1}^{2}\left(p_{j} f\left(z \mid \theta_{j}\right)\right\}^{v} \\
& \propto \mathrm{p}_{1}{ }^{\sum v_{i 1}}\left(\sigma_{1}\right)^{-\sum v_{11}} \exp \left\{-\sum_{i=1}^{n} \frac{v_{i 1}}{2 \sigma_{1}^{2}}\left(z_{i}-\mu_{1}\right)^{2}\right\} *\left(1-p_{1}\right)^{\sum v_{l 2}}\left(\sigma_{2}^{2}\right)^{\sum v_{b 02}} \exp \left\{-\sum_{i=1}^{n} \frac{v_{i 2}}{2 \sigma_{2}^{2}}\left(z_{i}-\mu_{2}\right)^{2}\right\}
\end{aligned}
$$


As distribuições a priori utilizadas foram:

(i) $\lambda, \alpha \sim \Gamma(a, b) \quad$ com a, b conhecidos

(ii) $\beta, \mu_{j} \sim N\left(c, d^{2}\right) \quad$ com c, d conhecidos

$(i) \sigma_{j}^{2} \sim I G(e, f) \quad$ com e, f conhecidos

(iii) $p \sim B(g, h) \quad$ com $\mathrm{g}, \mathrm{h}$ conhecidos

Com isso, temos o logaritmo das distribuições condicionais necessárias para o algoritmo ARMS,

$$
\begin{aligned}
& \text { (i) } l_{\alpha}=l_{\lambda}=l_{\mu_{\alpha}}=l_{\beta}=-\frac{1}{\sigma_{j}^{2}} \sum_{i=1}^{n} v_{i j}\left(z-\mu_{j}\right)^{2} \\
& \text { (ii) } l_{\sigma}=-\sum_{i=1}^{n} v_{i j} \ln (\sigma)-\frac{1}{\sigma^{2}} \sum_{i=1}^{n} v_{i j}\left(z-\mu_{j}\right)^{2}
\end{aligned}
$$

Observar que o algoritmo da Mistura de distribuições é mais complicado que o anterior. Em cada ciclo, gerar $W\left(\mathbf{x}_{1}\right), \ldots, W\left(\mathbf{x}_{n}\right)$ de uma distribuição Weibull na seguinte forma: gerar $t_{i}^{*}$ dado $x_{i}$ de uma distribuição Weibull com Função Distribuição Acumulada;

$$
F\left(t_{i} \mid \mathbf{x}_{i}\right)=1-\exp \left\{-\lambda \exp \left(\mathbf{x}_{\mathrm{i}}{ }^{\prime} \boldsymbol{\beta}\right) \mathrm{t}_{\mathrm{i}}^{\alpha}\right\}
$$

Com cada valor $t_{i}^{*}$ gerado calcular,

$$
\begin{aligned}
& W\left(x_{i}\right)=\exp \left\{-\ln S\left(t_{i}^{*} \mid \mathbf{x}_{i}\right)\right\} \\
& W\left(x_{i}\right)=\ln \left(\lambda^{0}\right)+\alpha^{0} \ln \left(t_{i}^{*}\right)+\mathbf{x}_{i} \boldsymbol{\beta}^{0}, \mathrm{i}=1,2, \ldots, \mathrm{n}
\end{aligned}
$$

Então, em cada etapa do algoritmo, atualizamos $W\left(\mathbf{x}_{1}\right), \ldots, W\left(\mathbf{x}_{n}\right), \beta_{1}, \ldots, \beta_{p_{1}}, \lambda_{1}$ $\alpha, \mu_{1}, \mu_{2}, \sigma_{1}, \sigma_{2}$ e p.

A previsão deste modelo é feita da mesma forma que a do modelo anterior, mudando apenas a forma de gerar $W\left(\mathbf{x}_{i}\right)$. 


\section{4 llustração Numérica}

Utilizamos o conjunto de dados 1, ou seja, os dados de Feigl e Zelen (1965) para ilustrar os modelos deste Capítulo. Assim, segundo a Tabela 3.1, temos os dados de 17 pacientes com câncer. Neste exemplo, temos apenas uma covariável e portanto, no nosso modelo, apenas $\beta_{1}$, já que $\beta_{0}$ será dado por $\lambda$.

Para uma análise Bayesiana utilizamos as distribuiçöes a priori dadas na seção de cada modelo. Os valores considerados para os hiperparâmetros foram: $a=1, b=1, c=0, d=1000, e=10, f=10, g=1, h=\sum v_{t}$.

Na Figura 6.1 abaixo, temos o gráfico de resíduos do modelo de riscos proporcionais ajustado. Nela podemos observar que um ajuste com mistura de distribuições normais no erro parece bastante razoável.

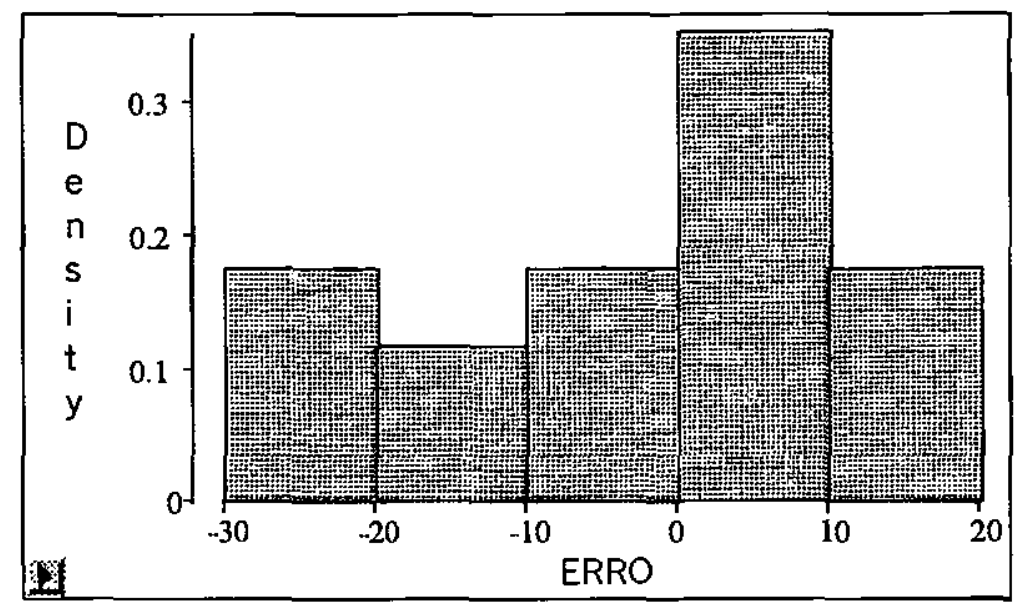

Figura 6.1: Gráfico da distribuição do erro no modelo de riscos proporcionais.

$\mathrm{Na}$ Tabela 6.1 temos as estimativas para os 3 modelos propostos. 
Tabela 6.1 Estimativas para os parâmetros.

\begin{tabular}{c|c|c|c}
\hline \multirow{2}{*}{ Modelo } & Parâmetro & $\begin{array}{c}\text { Média a } \\
\text { posteriori }\end{array}$ & $\begin{array}{c}\text { Intervalo de } \\
\text { Credibilidade } \\
(95 \%)\end{array}$ \\
\hline \multirow{3}{*}{$\begin{array}{c}\text { Riscos } \\
\text { Proporcionais }\end{array}$} & $\lambda$ & 3,759 & $(3,512 ; 4,188)$ \\
\cline { 2 - 4 } & $\beta_{1}$ & $-1,191$ & $(-1,387 ;-1,009)$ \\
\hline \multirow{3}{*}{$\begin{array}{c}\text { Efeitos } \\
\text { Aleatórios }\end{array}$} & $\alpha$ & 0,921 & $(0,900 ; 0,975)$ \\
\cline { 2 - 4 } & $\lambda$ & 3,591 & $(3,179 ; 4,027)$ \\
\cline { 2 - 4 } & $\beta_{1}$ & $-1,626$ & $(-1,978 ;-1,049)$ \\
\cline { 2 - 4 } & $\alpha$ & 1,026 & $(1,006 ; 1,098)$ \\
\hline \multirow{4}{*}{$\begin{array}{c}\text { Efeitos } \\
\text { cotón }\end{array}$} & $\beta_{1}$ & 1,2636 & $(0,887 ; 1,815)$ \\
\cline { 2 - 4 } Mistura & $\alpha$ & 3,998 & $(3,264 ; 4,359)$ \\
\cline { 2 - 4 } & $\beta_{1}$ & $-1,691$ & $(-1,861 ;-1,125)$ \\
\cline { 2 - 4 } & $\mu_{1}$ & 1,100 & $(0,986 ; 1,250)$ \\
\cline { 2 - 4 } & $\mu_{2}$ & $-19,16$ & $(-23,95 ;-17,78)$ \\
\cline { 2 - 4 } & $\sigma_{1}$ & 6,05 & $(2,63 ; 6,18)$ \\
\cline { 2 - 4 } & $\sigma_{2}$ & 7,01 & $(4,152 ; 8,798)$ \\
\cline { 2 - 4 } & $\mathrm{p}$ & 0,119 & $(0,003 ; 0,42)$ \\
\hline
\end{tabular}

Um meio interessante que pode ser utilizado para comparar esses modelos é através da previsão em cada um deles. Assim, poderemos ver qual deles se ajusta meihor a situação real.

Na Tabela 6.2 temos a previsão para cada um dos modelos. O Modelo1 é o de Riscos Proporcionais, o 2 o de Efeitos Aleatórios e o 3 é o de Efeitos Aleatórios com mistura. $\dot{E}$ interessante observar que os valores das estimativas para os parâmetros não diferem muito entre os modelos, o que nos leva a acreditar que elas estão coerentes. A estimativa do parâmetro $\beta_{1}$ foi a que mais se alterou, entretanto, entre os modelos de efeito aleatório e mistura a diferença foi pequena. 
Tabela 6.2 Previsão em cada modelo.

\begin{tabular}{c|c|c|c}
\hline $\mathbf{t}_{\mathbf{i}}$ & Modelo1 & Modelo2 & Modelo3 \\
\hline 65 & 54,493 & 59,090 & 64,496 \\
\hline 156 & 179,532 & 133,257 & 130,142 \\
\hline 100 & 97,978 & 94,147 & 108,744 \\
\hline 134 & 145,959 & 122,609 & 116,743 \\
\hline 16 & 8,077 & 15,359 & 14,367 \\
\hline 108 & 108,805 & 106,677 & 98,744 \\
\hline 121 & 127,020 & 119,518 & 122,350 \\
\hline 4 & 1,222 & 4,070 & 3,726 \\
\hline 39 & 27,177 & 37,196 & 40,560 \\
\hline 143 & 159,470 & 138,527 & 145,984 \\
\hline 56 & 44,483 & 55,099 & 51,075 \\
\hline 26 & 15,646 & 27,437 & 24,784 \\
\hline 22 & 12,462 & 23,307 & 21,023 \\
\hline 1 & 0,185 & 1,124 & 0,992 \\
\hline 1 & 0,185 & 1,124 & 0,992 \\
\hline 5 & 1,656 & 5,422 & 4,848 \\
\hline 65 & 54,493 & 73,094 & 64,496 \\
\hline & & &
\end{tabular}

Podemos observar pela Tabela que os modelos de Efeitos Aleatórios e Efeitos Aleatórios com mistura enriqueceram bastante a modelagem e ambos conseguiram fazer uma previsão bem mais próxima ao real que a feita pelo modelo de Riscos Proporcionais. 
Pela Figura 6.2 abaixo essa aproximação dos últimos modelos fica mais clara, já que fica mais fácil visualizá-la através do gráfico.

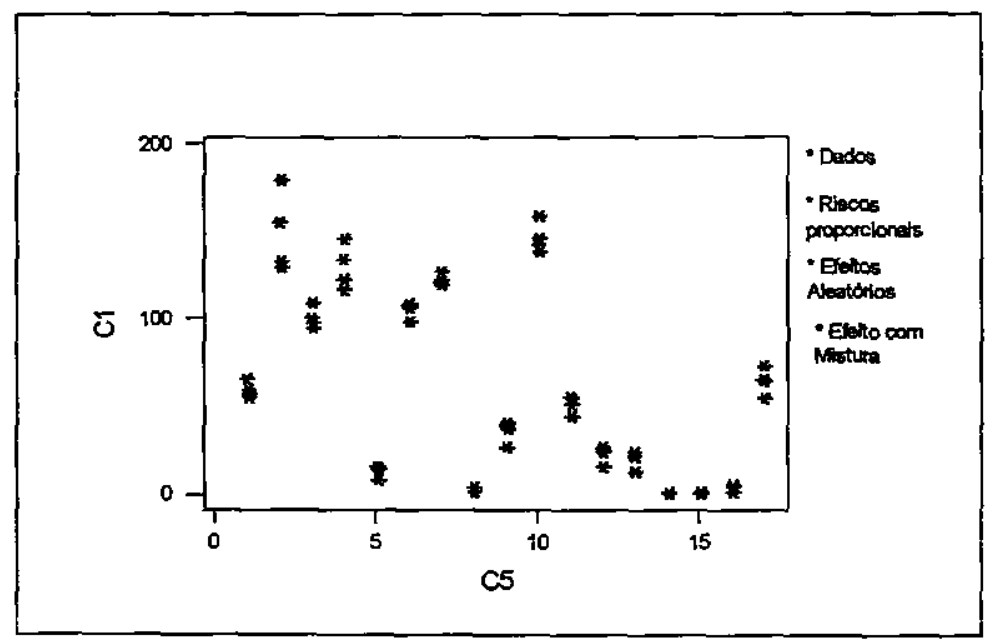

Figura 6.2: Gráfico das estimativas dos modelos 


\section{CAPÍTULO 7: Conclusões e Propostas Futuras}

A partir dos resultados encontrados, observamos várias vantagens do método Bayesiano usando algoritmos MCMC. Em primeiro lugar, no caso Bayesiano podemos encontrar melhores estimadores por intervalo do que usando aproximaçäo normal assintótica dos estimadores de máxima verossimilhança, especialmente com dados censurados.

Dificilmente temos dificuldade com os métodos de Monte Carlo. O uso dos software WinBugs e Ox facilitam muito a programaçăo no caso Bayesiano. Alguns programas ficam extremamente simples usando o WinBugs e o Ox nos ajuda muito em casos mais difíceis já que o tempo computacional é muito pequeno.

Outra vantagem Bayesiana é que através de métodos MCMC podemos selecionar os modelos de uma forma muito interessante usando a densidade preditiva ordenada que nos permite visualizar em que intervalos dada distribuição é melhor que as outras.

Além disso, no Capítulo 6, tivemos dificuldades em encontrar os EMV e uma análise Bayesiana foi feita sem maiores complicaçōes.

Vale lembrar que o SAS nos permitiu fazer análises clássicas com muita rapidez e com bastante facilidade também.

Como propostas futuras, algumas distribuiçōes a priori com menor variância podem ser incorporadas no modelo para melhorar os intervalos de credibilidade ou para verificar a influência delas nos modelos.

Seria interessante também tentar modelar mistura de distribuições para dados com várias covariáveis como por exemplo, os dados propostos por Ichida (1993), os quais consideram 154 pacientes com queimaduras. Neste caso temos mais de 15 convariáveis como : sexo do paciente, raça, porcentagem do corpo queimado, se existe queimadura nos troncos, tipos de queimaduras, etc. 
Além disso, os exemplos de modelos de Riscos Proporcionais podem ser estudados com maior ênfase em alguma trabalho futuro e assim confirmar a melhoria das estimativas com a introdução da variável $Z$, visto no Capítulo 6 . 


\section{ANEXO A}

Tabela A1: Dados de 90 pacientes com câncer na laringe.

\begin{tabular}{|c|c|c|c|c|}
\hline $\begin{array}{l}\text { Tempo de estudo } \\
\text { em meses }\end{array}$ & $\begin{array}{c}\text { Indicador } \\
1: \text { morte } \\
0: \text { censura }\end{array}$ & Estágio da Doença & $\begin{array}{c}\text { Idade no } \\
\text { Diagnóstico }\end{array}$ & Ano do Diagnóstico \\
\hline 0.6 & 1 & 1 & 77 & 76 \\
\hline 1.3 & 1 & 1 & 53 & 71 \\
\hline 2.4 & 1 & 1 & 45 & 71 \\
\hline 3.2 & 1 & 1 & 58 & 74 \\
\hline 3.3 & 1 & 1 & 76 & 74 \\
\hline 3.5 & 1 & 1 & 43 & 71 \\
\hline 3.5 & 1 & 1 & 60 & 73 \\
\hline 4.0 & 1 & 1 & 52 & 71 \\
\hline 4.0 & 1 & 1 & 63 & 76 \\
\hline 4.3 & 1 & 1 & 86 & 74 \\
\hline 5.3 & 1 & 1 & 81 & 72 \\
\hline 6.0 & 1 & 1 & 75 & 73 \\
\hline 6.4 & 1 & 1 & 77 & 72 \\
\hline 6.5 & 1 & 1 & 67 & 70 \\
\hline 7.4 & 1 & 1 & 68 & 71 \\
\hline 2.5 & 0 & 1 & 57 & 78 \\
\hline 3.2 & 0 & 1 & 51 & 77 \\
\hline 3.3 & 0 & 1 & 63 & 77 \\
\hline 4.5 & 0 & 1 & 48 & 76 \\
\hline 4.5 & 0 & 1 & 68 & 76 \\
\hline 5.5 & 0 & 1 & 70 & 75 \\
\hline 5.9 & 0 & 1 & 47 & 75 \\
\hline 5.9 & 0 & 1 & 58 & 75 \\
\hline 6.1 & 0 & 1 & 77 & 75 \\
\hline 6.2 & 0 & 1 & 64 & 75 \\
\hline 6.5 & 0 & 1 & 79 & 74 \\
\hline 6.7 & 0 & 1 & 61 & 74 \\
\hline 7.0 & 0 & 1 & 66 & 74 \\
\hline 7.4 & 0 & 1 & 73 & 73 \\
\hline 8.1 & 0 & 1 & 56 & 73 \\
\hline 8.1 & 0 & 1 & 73 & 73 \\
\hline 9.6 & 0 & 1 & 58 & 71 \\
\hline 10.7 & 0 & 1 & 68 & 70 \\
\hline 0.2 & 1 & 2 & 86 & 74 \\
\hline 1.8 & 1 & 2 & 64 & 77 \\
\hline 2.0 & 1 & 2 & 63 & 75 \\
\hline 3.6 & 1 & 2 & 70 & 77 \\
\hline 4.0 & 1 & 2 & 81 & 71 \\
\hline 6.2 & 1 & 2 & 74 & 72 \\
\hline 7.0 & 1 & 2 & 62 & 73 \\
\hline 2.2 & 0 & 2 & 71 & 78 \\
\hline 2.6 & 0 & 2 & 67 & 78 \\
\hline 3.3 & 0 & 2 & 51 & 77 \\
\hline 3.6 & 0 & 2 & 72 & 77 \\
\hline 4.3 & 0 & 2 & 47 & 76 \\
\hline 4.3 & 0 & 2 & 64 & 76 \\
\hline 5.0 & 0 & 2 & 66 & 76 \\
\hline
\end{tabular}


Tabela A1: Dados de 90 pacientes com câncer na laringe.

\begin{tabular}{|c|c|c|c|c|}
\hline 7.5 & 0 & 2 & 50 & 73 \\
\hline 7.6 & 0 & 2 & 53 & 73 \\
\hline 9.3 & 0 & 2 & 61 & 71 \\
\hline 0.3 & 1 & 3 & 49 & 72 \\
\hline 0.3 & 1 & 3 & 71 & 76 \\
\hline 0.5 & 1 & 3 & 57 & 74 \\
\hline 0.7 & 1 & 3 & 79 & 77 \\
\hline 0.8 & 1 & 3 & 82 & 74 \\
\hline 1.0 & 1 & 3 & 49. & 76 \\
\hline 1.3 & 1 & 3 & 60 & 76 \\
\hline 1.6 & 1 & 3 & 64 & 72 \\
\hline 1.8 & 1 & 3 & 74 & 71 \\
\hline 1.9 & 1 & 3 & 53 & 74 \\
\hline 1.9 & 1 & 3 & 72 & 74 \\
\hline 3.2 & 1 & 3 & 54 & 75 \\
\hline 3.5 & 1 & 3 & 81 & 74 \\
\hline 5.0 & 1 & 3 & 59 & 73 \\
\hline 6.3 & 1 & 3 & 70 & 72 \\
\hline 6.4 & 1 & 3 & 65 & 72 \\
\hline 7.8 & 1 & 3 & 68 & 72 \\
\hline 3.7 & 0 & 3 & 52 & 77 \\
\hline 4.5 & 0 & 3 & 66 & 76 \\
\hline 4.8 & 0 & 3 & 54 & 76 \\
\hline 4.8 & 0 & 3 & 63 & 76 \\
\hline 5.0 & 0 & 3 & 49 & 76 \\
\hline 5.1 & 0 & 3 & 69 & 76 \\
\hline 6.5 & 0 & 3 & 65 & 74 \\
\hline $\begin{array}{l}8.0 \\
\end{array}$ & 0 & 3 & 78 & 73 \\
\hline 9.3 & 0 & 3 & 69 & 71 \\
\hline 10.1 & 0 & 3 & 51 & 71 \\
\hline 0.1 & 1 & 4 & 65 & 72 \\
\hline 0.3 & 1 & 4 & 71 & 76 \\
\hline 0.4 & 1 & 4 & 76 & 77 \\
\hline 0.8 & 1 & 4 & 65 & 76 \\
\hline 0.8 & 1 & 4 & 78 & 77 \\
\hline 1.0 & 1 & 4 & 41 & 77 \\
\hline 1.5 & 1 & 4 & 68 & 73 \\
\hline 2.0 & 1 & 4 & 69 & 76 \\
\hline 2.3 & 1 & 4 & 62 & 71 \\
\hline 3.6 & 1 & 4 & 71 & 75 \\
\hline 3.8 & 1 & 4 & 84 & 74 \\
\hline 2.9 & 0 & 4 & 74 & 78 \\
\hline 4.3 & 0 & 4 & 48 & 76 \\
\hline
\end{tabular}




\section{ANEXO B}

\section{Critério de Convergência: Gelman \& Rubin (1992)}

Gelman e Rubin propuseram um teste de convergência baseado em 2 ou mais cadeias paralelas, cada uma começando com um valor inicial diferente. $O$ método é baseado na comparação de variâncias entre e dentre as cadeias para cada variável, o que é essencialmente uma análise de variância clássica. Essa comparação é usada para estimar o fator pelo qual o parâmetro de escala da distribuição marginal a posteriori para cada variável pode ser reduzido se a cadeira fosse infinita. Melhores resultados são obtidos para parâmetros cuja densidade marginal a posteriori é aproximadamente normal.

O critério de Gelman \& Rubin feito pelo CODA são os quantis $50 \%$ e $95 \%$, isto significa que somente a segunda parte da cadeia é usada para estimar esses quantis. Se ambas quantidades são aproximadamente 1.0, uma convergência efetiva é diagnosticada.

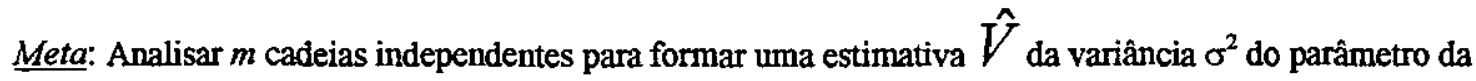
variável aleatória de interesse, dadas as observações simuladas até a presente iteração.

Seja $B / n$ a variancia entre as cadeias, definida por:

onde

$$
\frac{B}{n}=\frac{1}{m-1} \sum_{i=1}^{m}\left(\bar{\theta}_{i}^{\bullet}-\bar{\theta}_{\bullet}^{\bullet}\right)^{2}
$$

$$
\bar{\theta}_{i}^{\bullet}=\frac{1}{n} \sum_{t=n+1}^{2 n} \theta_{i}^{t} \quad \bar{\theta}_{i}^{\bullet}=\frac{1}{m} \sum_{i=1}^{m} \bar{\theta}_{i}^{\bullet}
$$

$\theta_{i}^{t}=\theta\left(X_{i}^{t}\right)$ é a $t$-ésima observação de $\theta$ a partir da cadeia $i$. Seja $W$ a variância dentro de cada cadeia, definida por: 


$$
W=\frac{1}{m} \sum_{i=1}^{m} s_{i}^{2}, \quad \text { tal que } s_{i}^{2}=\frac{1}{n-1} \sum_{t=n+1}^{m}(t
$$

Seja o estimador $\hat{V}$ definido por $\hat{V}=\frac{n-1}{n} W+\left(1+\frac{1}{m}\right) \frac{B}{n}$

Monitora-se a evolução de $\sqrt{\hat{R}}=\sqrt{\frac{\hat{V}}{W}}$

Se $\sqrt{\hat{R}} \approx 1 \Rightarrow$ Convergência 


\section{ANEXO C}

\section{Critério de Convergência: Geweke (1992)}

i) Assume-se que a cadeia foi simulada por $m+n$ iterações.

ii) $\operatorname{Seja} \theta^{(j)}=\theta\left(X^{()}\right)$, para $j=1, \ldots, n$, uma função real.

iii) A trajetória $\theta^{(1)}, \theta^{(2)}, \ldots$, define um série temporal e médias ergódicas para esta série podem ser calculadas.

As médias ergódicas são determinadas da seguinte forma:

$$
\bar{\theta}_{b}=\frac{1}{n_{b}} \sum_{j=m+1}^{m+n_{b}} \theta^{(j)} \quad \bar{\theta}_{a}=\frac{1}{n_{a}} \sum_{j=m+n-n_{a}+1}^{m+n} \theta^{(j)}
$$

onde $n_{b}+n_{a}<n$. Sugestão: $n_{a}=0,5 n$ e $n_{b}=0,1 n$.

À medida que $n$ cresce e as relações $\frac{n_{a}}{n}$ e $\frac{n_{b}}{n}$ são mantidas constantes, tem-se que:

$$
z_{G}=\frac{\bar{\theta}_{a}-\bar{\theta}_{b}}{\sqrt{\hat{\operatorname{Var}}\left(\theta_{a}\right)+\hat{\operatorname{Var}}\left(\theta_{b}\right)}} \rightarrow N(0,1)
$$

Assim, $z_{G}$ não deve ser grande ( $\left.>2\right)$ se a convergência foi alcançada.

Graficamente, tem-se

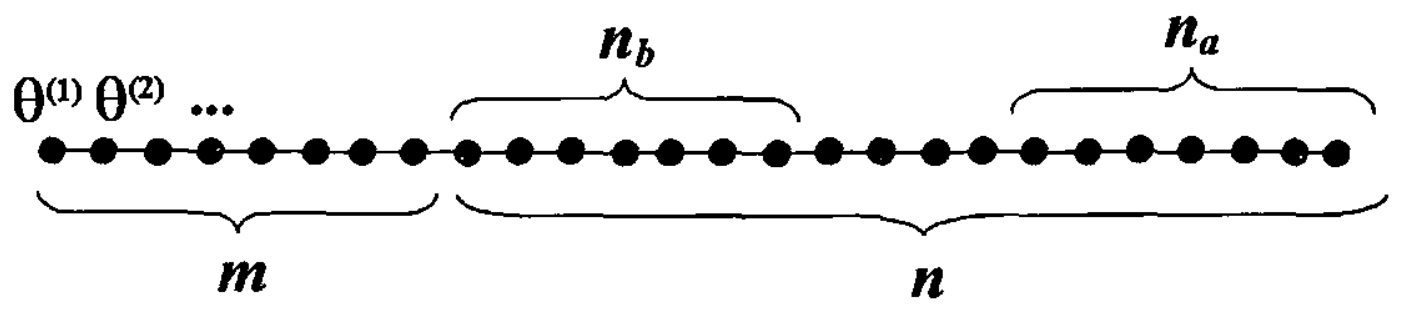

(burn-in) 


\section{Programa no software Matlab para o método Geweke:}

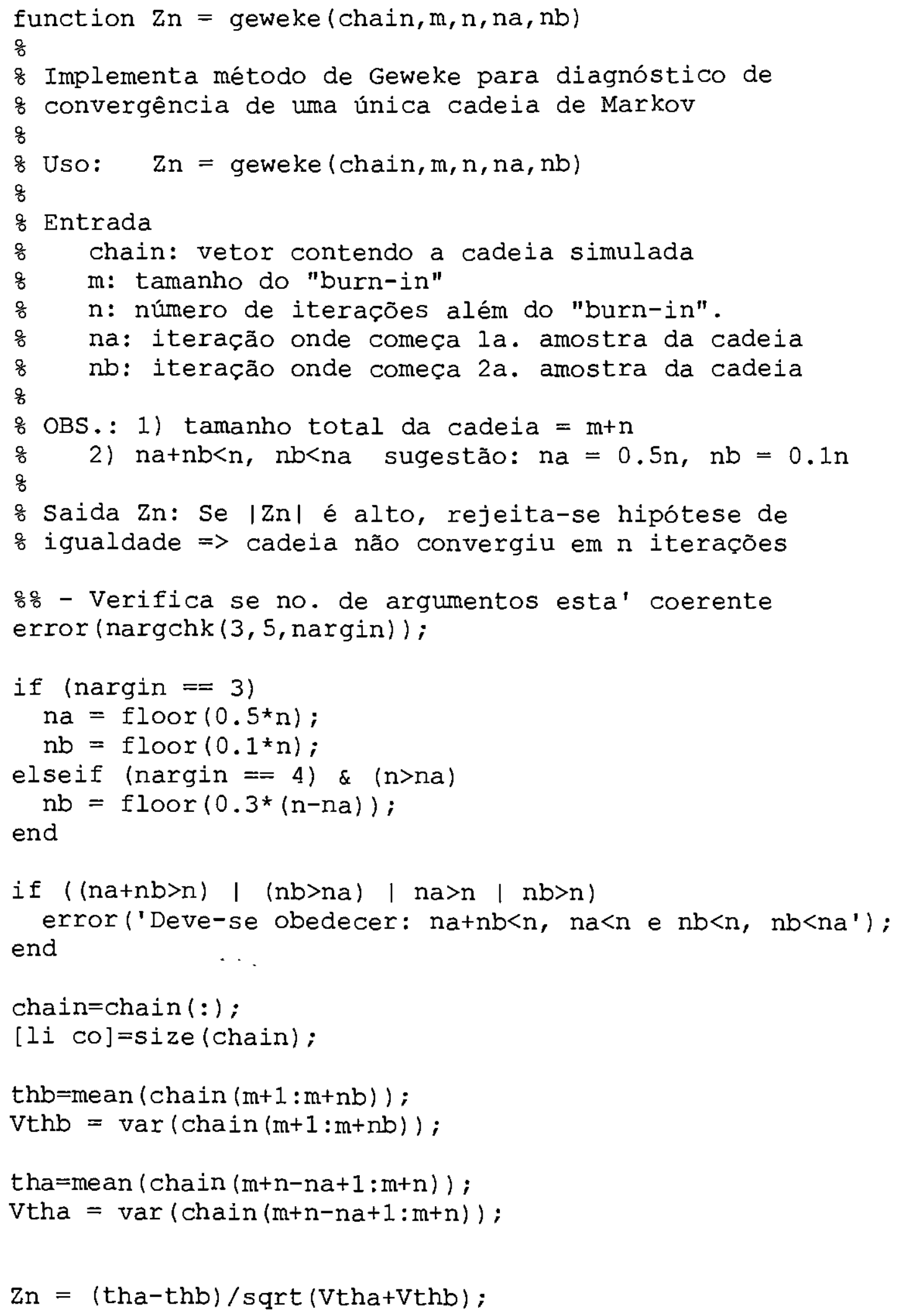




\section{BIBLIOGRAFIA}

Achcar, Jorge A., 1991, Inference for the Birbaum-Saunders fatigue life model using Bayesian methods, Computacional Statistics \& Data Analysis, 15,367-380.

Achcar, Jorge A., and Espinosa, Mariano M.,1991, Bayesian methods in accelerates lige tests considering a log-linear model for the Birbaum-Saunders distribution, Revista Brasileira de Estatística, 52,47-68.

Best, N.G.; Spiegelhalter, D.J.;Thomas, A.;Brayne, C. E. G. ,1996, Bayesian Analysis of Realistically Complex Models, Journal of the Royal Statistical Society, Series A, 159,323-342.

Box, G. E. P., Jenkins, G. M. and Reinsel, G. C., 1994, Time Series Analysis: Forecasting and Control, New Jersey, Prentice Hall, 3 edição.

Dieboit, J., and Robert, C., 1994, Estimation of finite mixture distributions through Bayesian sampling. Journal of the Royal Statistical Society, Seris B, 56, 363-375.

Doornik, J. A., 1999, Object-Oriented Matrix Programming Using Ox, $3^{\text {rd }}$ ed. London: Timberlake Consultants Press and Oxford.

Feigl, P., and Zelen, M., 1965, Estimation of exponencial survival probabilities with concomitant information. Biometrics, 21, 826-838.

Gehan, E. A., 1961, The determination of the number of patients required in a preliminary and follow-up trial of a news chemotherapeutic agent. J. Chronic Dis, $13,346-353$.

Gehan, E. A., 1965, A generalized Wilcoxon test for comparing arbitrarily singlycensored samples. Biometrika, 52, 203-223.

Gehan, E. A., and Lemak, N. A., 1994, Statistical in Medical Research. New York, Plenum Medical Book Company.

Gelfand, A. E., and Smith, A. F. M., 1990, Sampling based approaches to calculating marginal densities. Journal of the American Statistical Association, 85, 398-409.

Gelman, A., and Rubin, D. B., 1992, inference from iterative simulation using multiple sequences (with discussion). Statistical Science, 7, 457-511. 
Gilks, W.R., Best, N.G. e Tan, K.C. 1995, Adaptive rejection Metropolis sampling whithin Gibbs sampling. Journal of the Royal Statistical Society, Series' C, 44:455472.

Kardaun, O., 1983, Statistical Analysis of Male Larynx-Cancer Patients-Acase. study. Statistical Nederlandica, 37, 103-126.

Klein, J.P., Moeschberger, M. L. 1997, Survival Analysis: Techniques for Censored and Truncated Data, Springer-Veriag, New-York.

Krall, J., Uthoff V., and Hariey J., 1975, A step-up procedure for selecting variables associated with survival. Biometrics, 31, 49-57.

Lawless, J. F., 1982, Statistical Models and Methods for Lifetime Data. New York, John Wiley \& Sons.

Mantel, N., and Haenszel, W., 1959, Statistical aspects of the analysis of data from retrospective studies of disease. J Natl Cancer Inst, 22, 719-748.

Mantel, N., 1966, Evaluation of survival data and two new rank order statistics arising in its consideration. Cancer chemother Rep, 50, 163-170.

Nelson, W., B., and Mahn, S., J., 1972, Linear estimation of a regression relationship from censored data. Part 1 - Simple methods and their applications.

Smith, A. F. M., and Roberts, G. O.,1993, Bayesian computation via the Gibbs Sampler and related Markov
Statistical Society, B, 55, 3-24.

Tanner, M. A., and Wong, W. H. 1987, The calculation of posterior distributions via data argumentation (with Finite Mixtur A., and Altschuler, B., 1976, Lung cancer incidesicens. Biometrics, $32,805-816$. 
Gilks, W.R., Best, N.G. e Tan, K.C. 1995, Adaptive rejection Metropolis sampling whithin Gibbs sampling. Journal of the Royal Statistical Society, Series C, 44:455472.

Kardaun, O., 1983, Statistical Analysis of Male Larynx-Cancer Patients.-Acase study. Statistical Nederlandica, 37, 103-126.

Klein, J.P., Moeschberger, M. L. 1997, Survival Analysis: Techniques for Censored and Truncated Data, Springer- Verlag, New-York.

Krall, J., Uthoff V., and Harley J., 1975, A step-up procedure for selecting variables associated with survival. Biometrics, 31, 49-57.

Lawless, J. F., 1982, Statistical Models and Methods for Lifetime Data. New York, John Wiley \& Sons.

Mantel, N., and Haenszel, W., 1959, Statistical aspects of the analysis of data from retrospective studies of disease. J Natl Cancer Inst, 22, 719-748.

Mantel, N., 1966, Evaluation of survival data and two new rank order statistics arising in its consideration. Cancer chemother Rep, 50, 163-170.

Nelson, W., B., and Mahn, G., J., 1972, Linear estimation of a regression relationship from censored data. Part 1 - Simple methods and their applications. Technometrics, 14, 247-269.

Smith, A. F. M., and Roberts, G. O.,1993, Bayesian computation via the Gibbs Sampler and related Markov chain Monte Carlo methods. Journal of the Royal Statistical Society, B, 55, 3-24.

Tanner, M. A., and Wong, W. H., 1987, The calculation of posterior distributions via data argumentation (with discussion). Journal of the American Statistical Association, 82, 528-550.

Titterington, D. M., Smith, A. F. M. and Makov, U. E., 1985, Statistical Analysis of Finite Mixture Distributions, New York, John Wiley \& Sons.

Whittemore, A., and Altschuler, B., 1976, Lung cancer incidence in cigarette smokers: further analysis of Doll and Mill's data for British physicians. Biometrics, 32, 805-816. 